\title{
INTUITIVE MYOELECTRIC CONTROL OF UPPER LIMB PROSTHESES
}

\author{
Dissertation \\ zur Erlangung des mathematisch \\ naturwissenschaftlichen Doktorgrades \\ "Doctor rerum naturalium" \\ der Georg-August-Universität Göttingen \\ im Promotionsprogramm PCS \\ der Georg-August University School of Science (GAUSS)
}

Vorgelegt von

Dipl.-Ing. Dipl.-Wirt.Ing.

Hubertus Rehbaum

aus Warendorf

Göttingen, 2014

Academic Supervisors:

Prof. Dr. Dr. Dario Farina

Prof. Dr. Otto Rienhoff 
Referent: $\quad$ Prof. Dr. Dr. Dario Farina, Department of Neurorehabilitation Engineering Korreferent: Prof. Dr. Otto Rienhoff, Institut für Medizinische Informatik

\section{Mitglieder der Prüfungskommission}

Prof. Dr. Jens Grabowski

Prof. Dr. Dieter Hogrefe

Prof. Dr. Ulrich Sax

Prof. Dr. Ramin Yahyapour 


\section{Preface}

In this thesis I present the results of my work at the Otto Bock HealthCare GmbH Duderstadt and the Universitätsmedizin Göttingen within the EU funded IAPP project AMYO, project reference 251555, project duration 01. September $2010-31$. August 2014.

All studies involving human subjects were approved by the „Ethikkommission der Universitätsmedizin Göttingen“.

All chapters of this thesis are original and written by me. Only subchapter 2.1 has been adapted from my own manuscript I submitted to the Springer ${ }^{\circledR}$ journal „Medical \& Biological Engineering \& Computing" and which is currently under review.

All graphics in this manuscript are original and created solely by myself. However, some of the graphics have also been submitted or published in modified versions in manuscripts I contributed to as an author.

Hereby I declare that I have written this thesis independently and with no other aids and sources than quoted.

Göttingen, $6^{\text {th }}$ March 2014

Hubertus Rehbaum 


\section{$\underline{\text { Abstract }}$}

The myoelectric control of hand prosthesis commercially available is simple and limits the user to very basic operations. Although in the academic research for prosthesis control a large variety of advanced control methods has been developed, none of them has replaced the current industrial state of the art, yet. In this PhD project I have investigated and developed an approach towards intuitive prostheses control, based on new signal-processing and regression algorithms. By introducing a novel adaptive pre-processing algorithm (ACAR) for the surface EMG signals and designing a regression system based on a non-negative matrix factorization, I have developed a myocontrol system capable of online control of upper limb prosthesis for two degrees of freedom, simultaneously and proportionally. Additionally, I have developed a virtual evaluation paradigm, which can assess the control performance of important hand movements necessary for daily life activities. This online assessment goes beyond the state of the art of myoelectric control research, which is done offline. That is without the interaction with the subject.

The resulting myocontrol system and virtual evaluation paradigm have been tested in both intact-limb subjects and subjects with limb deficiencies. In these studies, the benefits of the developed algorithms have been confirmed. The scientific results and developments of this project have been the basis for additional publications and scientific achievements by the Department of Neurorehabilitation Engineering and its scientific partners. This underlines the impact of this work in the field of myoelectric control for upper limb prostheses. 


\section{Acknowledgements}

Above all, I thank my supervisor Prof. Dr. Dario Farina for the continuous support during my $\mathrm{PhD}$ project. The fruitful discussions and his guidance have been an invaluable input for my thesis. I also thank Prof. Dr. Otto Rienhoff for his support both during the application phase as well as throughout the PhD project. Without him I would not have been able to enroll as a student at the University of Göttingen.

Furthermore, I thank the Department of Neurorehabilitation Engineering, especially the myocontrol group around Dr. Ning Jiang.

I thank PD Dr. Bernhard Graimann, the Otto Bock Healthcare GmbH and the Otto Bock Healthcare Products GmbH for providing me the opportunity to join the AMYO project as an early stage researcher and for supporting me also during my secondment at UMG.

I thank Ivan Vujaklija for being a great colleague and even greater friend.

I thank my family for providing me the means and the support to get this far.

For Liliana, I'm lucky to have you. 


\section{Contents}

Preface

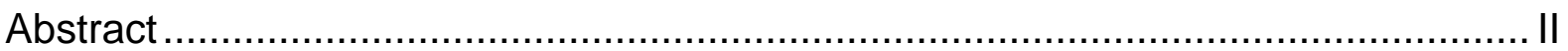

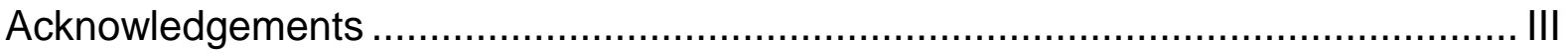

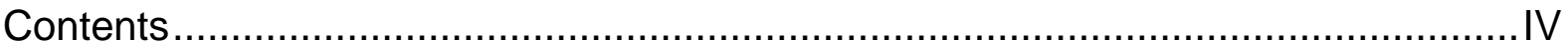

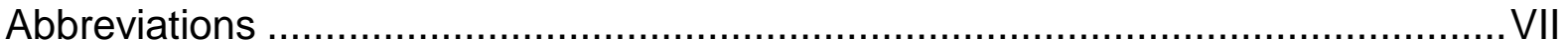

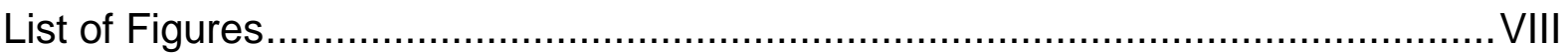

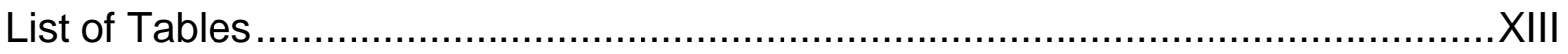

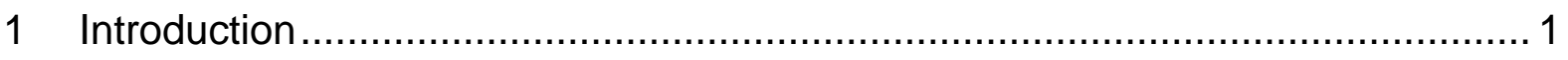

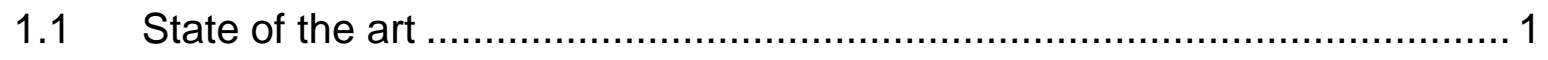

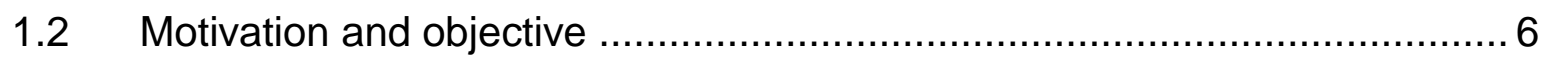

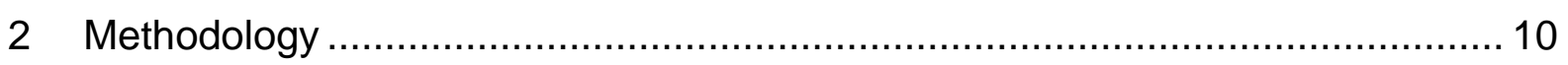

2.1 Adaptive Common Average Reference Filtering ................................... 11

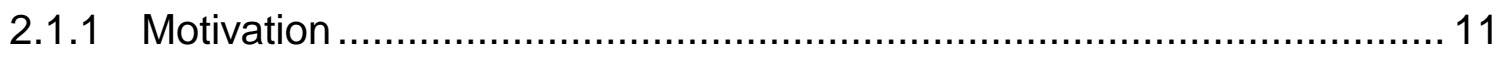

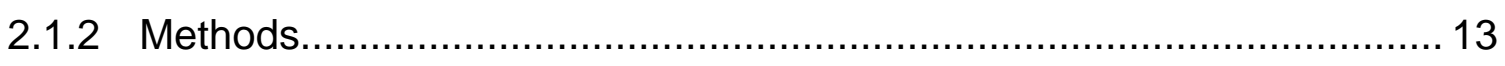

2.1.2.1 Adaptive common average reference (ACAR) ............................. 13

2.1.2.2 Quantitative evaluation of noise reduction and selectivity .............. 15

2.1.2.3 Impact on myocontrol based on pattern classification .................... 16

2.1.2.4 Impact on regression based myocontrol systems …..................... 17

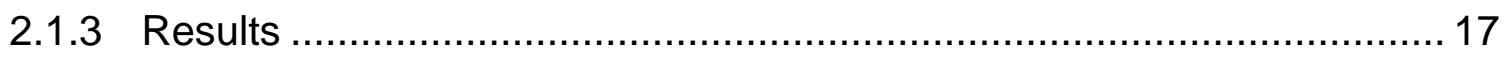


2.1.3.1 Quantification of noise reduction and selectivity

2.1.3.2 Myocontrol based on pattern classification 19

2.1.3.3 Discussion 20

2.1.3.4 Conclusion 21

2.2 Virtual Evaluation Paradigm .............................................................. 23

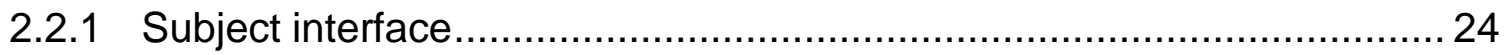

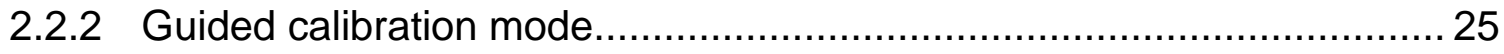

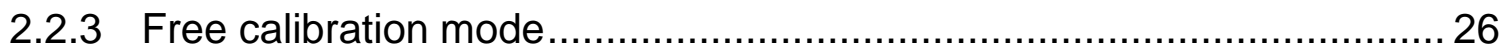

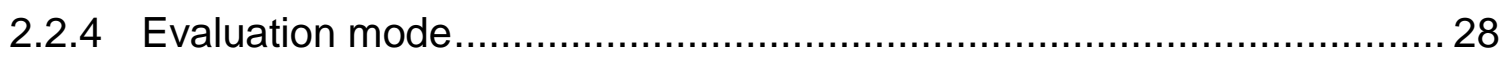

2.2.5 Performance assessment task .................................................... 29

2.2.6 Performance metrics provided by the performance assessment task ....33

2.2.6.1 Task completion rate .................................................... 33

2.2.6.2 Task completion time and path efficiency.............................. 33

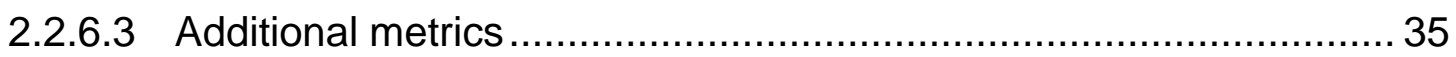

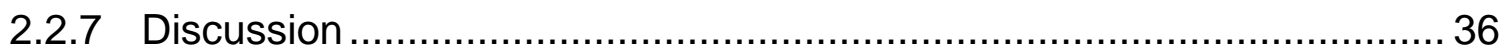

2.3 Online Regression Control System ............................................. 38

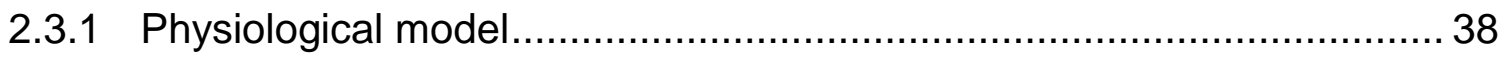

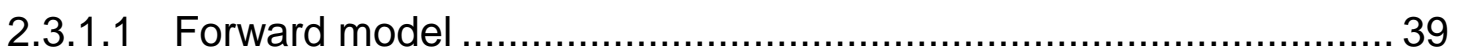

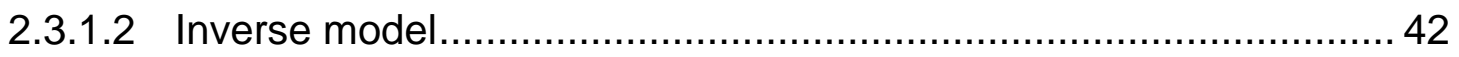

2.3.2 Structure of the developed control system ..................................... 42

2.3.2.1 EMG acquisition system ................................................... 43 
2.3.2.2 Estimation of the muscle activation from the surface EMG

2.3.2.3 Calibration data 46

2.3.2.4 Extracting the synergy matrix using Non-negative Matrix Factorization 47

2.3.2.5 Estimation of control signals using the synergy matrix 52

3 Experimental studies on the new myocontrol scheme and results. 58

3.1 Study 1: Impact of the ACAR filter on the Online Regression Control System 58

3.1.1 Methods 58

3.1.2 Results 59

3.1.3 Study discussion 60

3.2 Study 2: Evaluation of the Online Regression Control System 61

3.2.1 Methods 61

3.2.2 Results 64

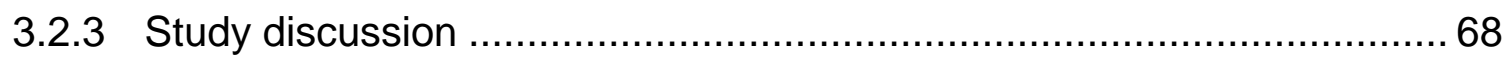

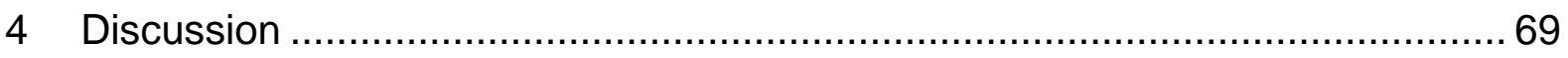

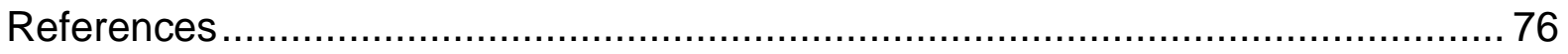

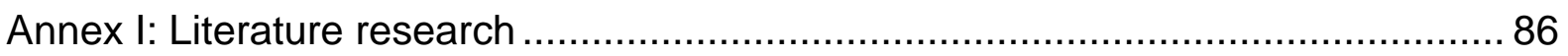

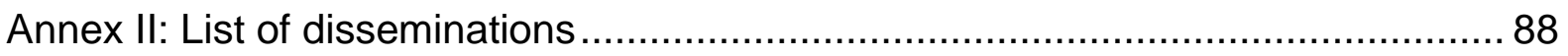




\section{$\underline{\text { Abbreviations }}$}

ACAR Adaptive Common Average Reference

CNS Central nervous system

CS Coordinate System

DOF Degree of freedom

EMG Electromyography

NMF Non-negative Matrix Factorization

VEP Virtual Evaluation Paradigm 


\section{List of Figures}

Figure 1: The first myoelectric prostheses used only a single EMG channel (a) recoded from a muscle site. Using two different thresholds, the hand could be opened or closed. The current industrial state of the art uses two electrode sites (b), providing also a proportional control. (This graphic has been published by the author in [109])

Figure 2: Examples of myoelectric hands commercially available: The Ottobock DMC Plus (a) is a typical prosthetic device with hand open/close and rotation that is widely used in clinical practice. In contrast to this the Ottobock Michaelangelo Hand (b), Touchbionics iLimb (c), and RSL Steeper Bebionic (d) are more advanced prosthetic hands, providing additional functions like the independent control of single fingers. Although the four models shown differ in complexity and components, with some offering different grip patterns (adjustable by external means), all are based on the simple and sequential control scheme, including its limitations for the prosthetic user.

Figure 3: Comparison of the necessary steps to pour water into a glass from a bottle, using the industrially available, conventional control (a) and the currently in academia used pattern recognition approach (b). (This graphic is an original work and has also been published in a modified version in [116])

Figure 4: Basic signal processing chain for pattern recognition based control. The recorded multichannel surface EMG can be pre-processed (band pass, notch filter), followed by a windowing and feature extraction stage. Depending on the application, a dimensionality reduction can be performed. Finally, the extracted features are used as input for the classification algorithm, resulting in a sequence of detected gestures as the output of the system.

Figure 5: (a) Raw multi-channel EMG signal (16 monopolar channels). (b) The same signal filtered using the classic common average reference (CAR) method. For the first EMG burst (approximately from 1s to $2 \mathrm{~s}$ of the recording) signal components are added by CAR in channels 1-4, which are originally not active. A similar effect is visible for the second burst of activity for channels 5-16.

Figure 6: Comparison of the different selection criteria for the ACAR algorithm. These results are obtained from subject 3 and show that all methods exceed the SNRs of the standard CAR. The same results were found for the other subjects. 
Figure 7: Comparison of the SNRs using K/2 criteria and different window length $L$ for subject 3 . The results show that a longer window length has a stronger impact to the between-channel SNR, therefore increasing the separability. Similar results were found for the other selection criteria and subjects.

Figure 8: The results of the offline analysis for the four preprocessing methods are shown grouped by the four subjects (S1-4). For the ACAR the window length chosen was 25 samples with the $\mathrm{K} / 2$ channel selection criteria. The bars indicate the mean values over the four measures for each subject, with the standard deviation shown on top.

Figure 9: Projection of the LDA spaces for subject 3 onto the first two components, separated by the three input signals. Only the unfiltered monopolar signal and the CAR and ACAR filtered data was used. (Note: the three plots have the same axis scaling)

Figure 10: The three performance metrics (MSA, SI, DCOFF) have been calculated for all subjects and the three different preprocessing methods. The figure depicts the mean values and standard deviations for the four subjects. For better comparison, the values have been normalized on subject base using the results for unfiltered data (orange) as reference.

Figure 11: (a)The user interface of the Virtual Evaluation Paradigm (VEP) developed for online evaluation. The flexion and extension of the wrist is represented through the horizontal position of the arrow (green), the wrist rotation by the rotation of the arrow. The vertical markers indicate maximum flexion and extension. The rotation is limited by $+/-90^{\circ}$. (b) The position of the arrow's tip $\left(x_{\text {tip }}(t), y_{\text {tip }}(t)\right)$ can be calculated from the two control signals $(v(t), \varphi(t))$, using the equations provided.

Figure 12: Mapping of the arrow to the hand gestures for single DOF movements. This figure represents the subject's view when looking at the screen, with the hand pointed forward. The example is given for a right handed subject. For left handed subjects pronation and supination are swapped, as well as wrist flexion and extension.

Figure 13: During the Guided Calibration Mode the VEP is controlled by an external prompt generator. The subject follows this cue and the EMG is recorded synchronously with the presented prompt as labels. This labeled data can be used to calibrate the control system.

Figure 14: The Free Calibration Mode allows the subject to perform voluntary movements. Using three XSens measurement units the wrist angles are calculated and recorded synchronously with the EMG data. ....27 
Figure 15: In the Evaluation Mode the output of the calibrated control system is used to control the arrow in VEP.

Figure 16: Examples for the targets in the Performance Assessment Task. The target types are presented in different colors. The magenta targets can be reached using solely wrist flexion/extension. The orange targets only require wrist pronation/supination. Finally, the blue area indicates the area that can be reached using both DOF simultaneously. An example for these combined targets is presented in blue. . 30

Figure 17: The subject is asked to steer the tip of the arrow into the target circle (blue). The trajectory depends on the strategy chosen. In (a), a simultaneous activation of the two DOF is used, resulting in the shortest path from the starting point to the target. In contrast to this, a sequential path is presented in (b), a valid alternative but not optimal completion of the task.

Figure 18: Example for a task set, defining a run. In this run, six tasks are defined for each target type 1 (1a-1f) and target type 2 (2a-2f). For the target type 3 requiring combined activation of the two DOF, a total of 12 tasks is defined (3a-3I).

Figure 19: The path efficiency is calculated as a ratio between optimal path length and measured trajectory path length

Figure 20: Sagittal cut though the proximal third of the human forearm, showing the presence of 13 muscles in this area. (original work, previously used in [115]) 38

Figure 21: Forward synergy model for the control of wrist flexion/extension and wrist pronation/supination.

From the motor cortex the low dimensional motor commands (primitives) are send out to the spinal cord, where they are transformed into the high dimensional muscle activation signals, necessary to execute the intended movement. Finally, the muscle activation is recorded by the multi-channel surface EMG system. 40

Figure 22: Inverse model to extract the myocontrol signals $C$ from the recorded EMG signals $E$

Figure 23: The recording setup used for the EMG signal acquisition. Sixteen electrodes of type Ambu Neuroline 720 (see lower right box) were placed around the forearm in a 2x8 layout. The monopolar signals were acquired and digitalized by a EMG-USB2 amplifier. Finally, the digitalized signal was conditioned to represent the muscle activation in the synergy model (the forward model). 
Figure 25: The input of the regression system is the muscle activation estimation, provided by the signal conditioning unit. The core of the regression system is the mixing matrix, calculated from the calibration data. Using this matrix, the primitives were extracted from the muscle activation.

Figure 26: Resolving the indeterminacy for DOF 1: The label $v(t)$ for the recorded data is separated into the two function components $v^{+}(t)$ and $v^{-}(t)$. Using the cross correlation between these components and the extracted primitives the correct permutation matrix is identified.

Figure 27: Online processing chain to estimate the control signals. New EMG data is acquired by the provided hardware and the muscle activation is estimated using the ACAR filter and the EMG linear envelope. The resulting muscle activation is multiplied with the inverse synergy matrix and the resulting primitives are scaled using the previously identified scaling factors. Finally, the control signals are down sampled and the difference is calculated.

Figure 28: Structure of the non-linear post-processing. In the first component, the estimate is sanitized using a threshold and clipping stage. Weak activations close to zero are suppressed and overshoots are clipped. Only if the estimates are within the borders, they pass proportionally. The second component is an FIR filter to stabilize the output. Finally, the optional integrator as third component can be used to switch from position control mode to velocity control mode.

Figure 29: Acquired metric data of the online regression myocontrol using NMF. The left plot (a) shows the completion rate for the 20 targets of the four subjects using the three different EMG preprocessing methods in randomized order. The corresponding mean time to reach the targets and standard deviation is given in the right plot (b).

Figure 30: Prompt sequence used for the calibration phase of study 2 . The subject was presented with four dynamic activations within the range of motion for both DOF. Each trial was triggered manually by the experimenter. Therefore, the time between two repetitions (dotted sections) varied depending on the subject's preference.

Figure 31: Metrics for all subjects acquired with the Performance Assessment Task. For each subject, the mean and standard deviation for each metric are reported for the full run (wide light blue bars) as well as for each target type separately (thin color bars magenta, orange and dark blue on top of the light blue bars). Note the legend provided in the lower right for the detailed description of the plot. The completion rate 
(a) reports the number of targets that were successfully completed per subject. The mean and standard deviation for the completion time (b) and path efficiency (c) are calculated on the basis of the successfully completed targets only.

Figure 32: The metric overshoots (a) counts the number of times that the subject positioned the tip of the arrow onto the target circle, but did not manage to remain on the target for the required dwelling time. The speed (b) measures the average moving speed of the arrow's tip. 


\section{List of Tables}

Table 1: Basic performance metrics for the Performance Assessment Task ............................................... 33

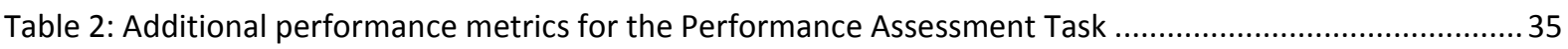

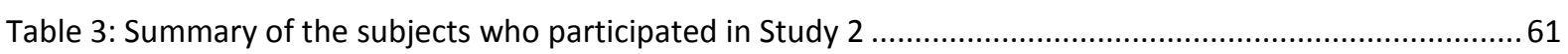

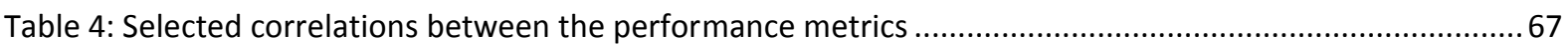

Table 5: $\mathrm{p}$-Values of the Kruskal-Wallis tests for the five performance metrics ..........................................68 


\section{Introduction}

\subsection{State of the art}

For humans, the hand is the most powerful tool available. It has more than 20 degrees of freedom (DOF) for the movement of the fingers, thumb, and wrist. This flexibility permits the execution of strong forces, like squeezing or holding heavy object, as well as the performance of precise and fine movements, such as threading a needle.

The loss of the hand through traumatic amputation or other accidents has a major impact on an individual. Therefore, approaches for restoring a lost limb are found early in time. Examples of replacement methods are indeed found around 300 B.C. in Egypt. The Götz von Berlichingen's Iron Hand, as a technological solution, dates back to the early $16^{\text {th }}$ century [1].

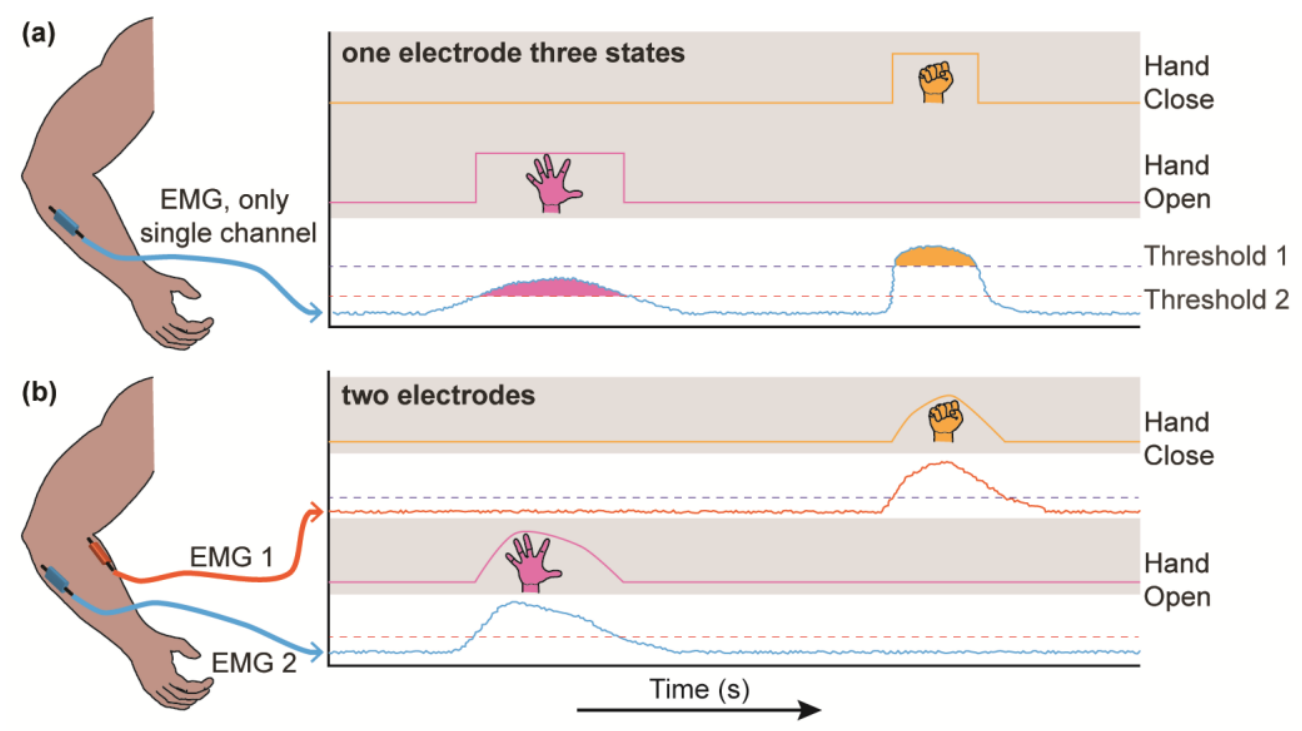

Figure 1: The first myoelectric prostheses used only a single EMG channel (a) recoded from a muscle site. Using two different thresholds, the hand could be opened or closed. The current industrial state of the art uses two electrode sites (b), providing also a proportional control. (This graphic has been published by the author in [109]) 
These inventions were succeeded by other mechanical body-powered hand prostheses, most of which used bicycle cable to activate the hand or a hook. Only by 1915 the first externally powered hand prosthesis was invented using compressed gas [2]. In the 1940's, Reiter introduced the idea of the first myoelectric prosthesis [3], [4]. Subsequently, similar designs were created independently in England, Canada, and the Soviet Union [5]-[11]. In the following years the myoelectric control of upper limb prostheses developed and different control concepts emerged, using first one electrode system with two thresholds (Figure 1a) [9] and, afterwards, two electrode systems to control the opening and closing of the hand (Figure 1b) [8], [11].

Since the 1960s, the mechatronic properties of the hand prostheses have substantially evolved. Reduced battery size, smaller actuators and better materials have resulted in more powerful, longer lasting, and more robust prosthetic devices [12]. Examples of current commercially available myoelectric hands are provided in

(a)

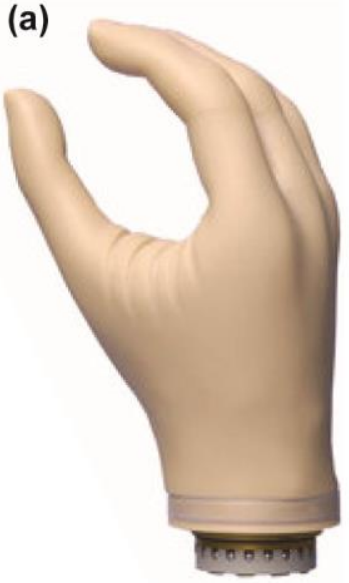

(b)

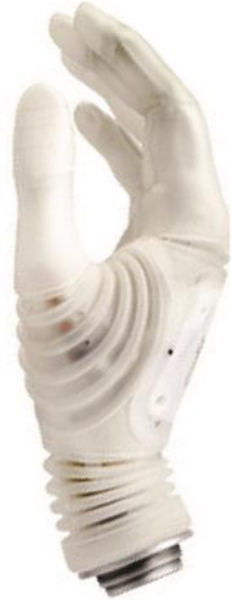

(c)

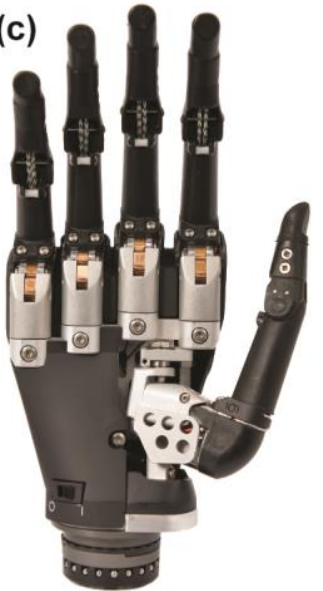

(d)

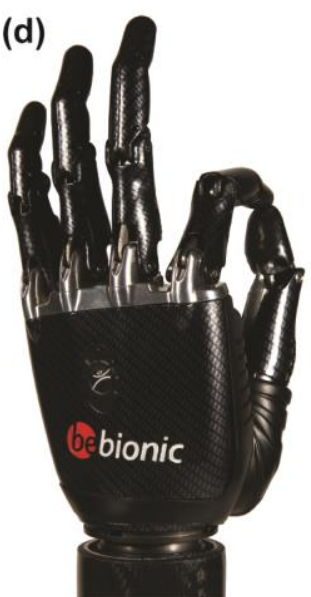

Figure 2: Examples of myoelectric hands commercially available: The Ottobock DMC Plus (a) is a typical prosthetic device with hand open/close and rotation that is widely used in clinical practice. In contrast to this the Ottobock Michaelangelo Hand (b), Touchbionics iLimb (c), and RSL Steeper Bebionic (d) are more advanced prosthetic hands, providing additional functions like the independent control of single fingers. Although the four models shown differ in complexity and components, with some offering different grip patterns (adjustable by external means), all are based on the simple and sequential control scheme, including its limitations for the prosthetic user. 
Figure 2. Nevertheless, the simple and sequential control scheme with two electrode sites is still the state of the art in commercially available prostheses (Figure 1b).

This conventional control scheme uses two electrodes placed independently on two muscle sites, resulting in two control signals, to control the grip and the rotation function of the prosthetic hand (two function modes or DOFs). One recording system is often placed on the flexor muscle group of the wrist, and the other is placed on the wrist extensor muscle group [8]. Using the activation of these two muscle sites, only one DOF can be controlled at a time to open and close, or to rotate the hand in supination or pronation. Additionally, a co-contraction is used to switch between the two function modes. The example of grabbing a water bottle to pour water into a glass is visualized in Figure 3a. After identifying which of the two DOFs is currently active, the hand has to be rotated into the correct position to grasp the bottle. After a mode switch into hand open/close function, the hand has to be opened enough to be able to engage around the bottle with the fingers, to then be closed again and thus grab the bottle. With the bottle in the hand, again a mode switch into rotation is

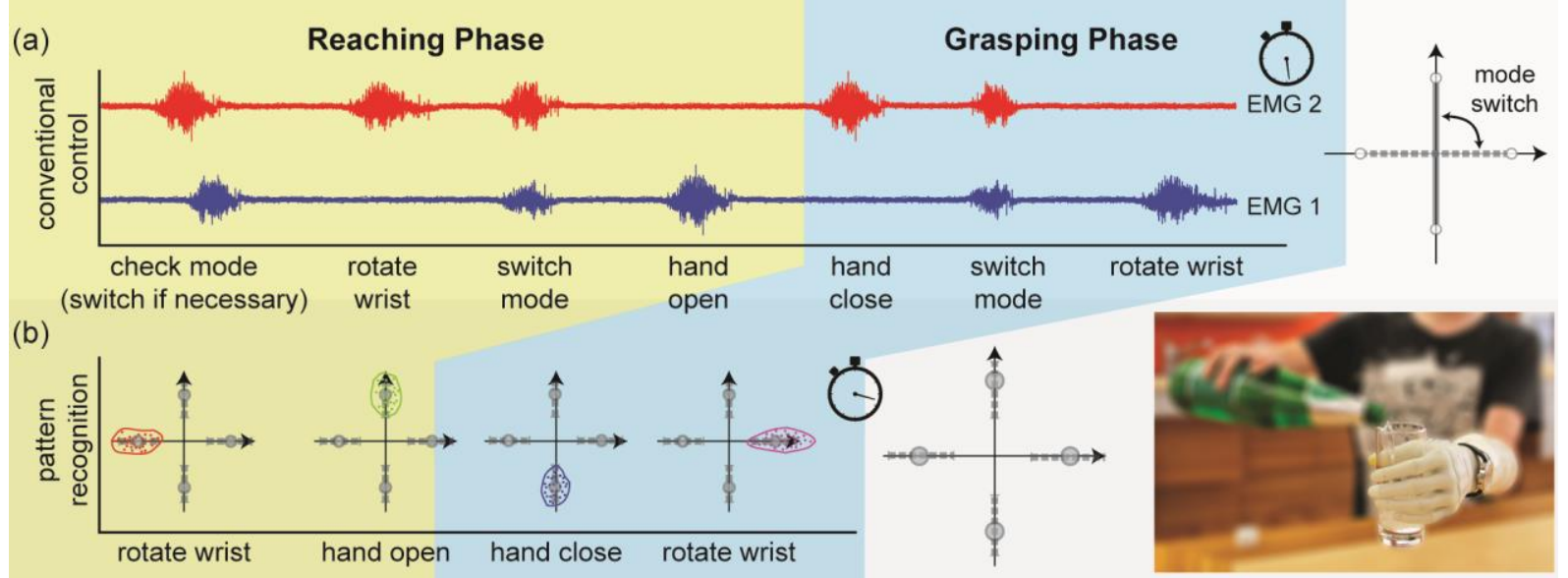

Figure 3: Comparison of the necessary steps to pour water into a glass from a bottle, using the industrially available, conventional control (a) and the currently in academia used pattern recognition approach (b). (This graphic is an original work and has also been published in a modified version in [116]) 


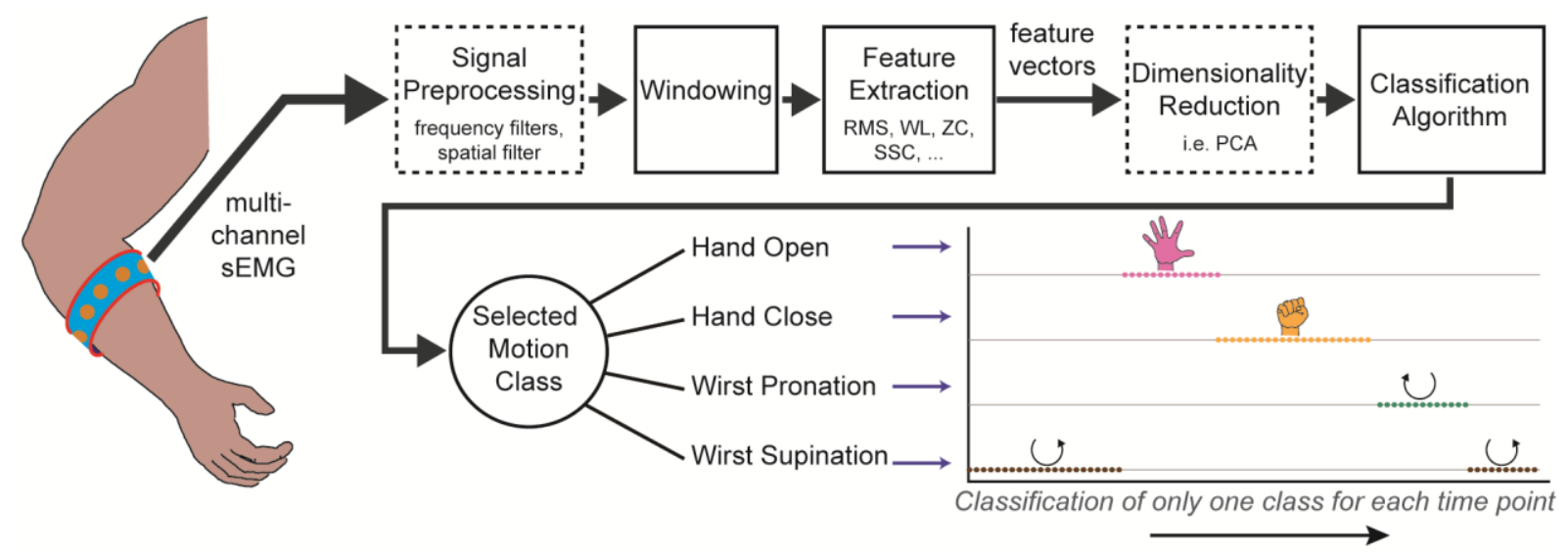

Figure 4: Basic signal processing chain for pattern recognition based control. The recorded multichannel surface EMG can be pre-processed (band pass, notch filter), followed by a windowing and feature extraction stage. Depending on the application, a dimensionality reduction can be performed. Finally, the extracted features are used as input for the classification algorithm, resulting in a sequence of detected gestures as the output of the system.

necessary to be able to pour the water into the glass.

For the industrial state of the art prosthesis control, this task requires a minimum of 6 steps and much of the control burden is left to the user with cumbersome, sequential and unnatural control strategies.

Academic research has indeed been developing powerful algorithms with promising results (pattern recognition, regression and non-linear transformations systems [13][16]), but there are still no commercial systems available using these approaches because they are not reliable enough for clinical applications. In contrast to the conventional control, pattern recognition provides the possibility of recognizing different hand gestures using surface EMG signals. These developments started as early as the 1970s [17]-[19].

In 1993, Hudgins et al presented a multi-functional myocontrol system [20] which became the classic multi-functional EMG pattern recognition method in this field [21]-[23]. This work introduced a filtering of the raw EMG signals and the extraction of a set of time domain features from the filtered EMG signals. Afterwards, a linear 
discriminant analysis (LDA) method classifies the features that correspond to a hand gesture to generate a command that drives the prosthesis. A representative version of this method is depicted in Figure 4.

The pattern recognition concept is based on the idea that multiple control signals/patterns can be extracted from multiple muscle sites in the forearm, making the use of co-contraction for mode switching obsolete. The myocontrol system based on pattern recognition has to first learn, in a training phase, the different patterns for the various hand functions and afterwards, during the clinical usage, the user's intention is determined by the pre-trained system and used to drive the prosthesis.

Pattern recognition allows for a continuous and multi-functional control, but imposes two major limitations. First, the different motion intentions can only be detected in a sequence of single activations; therefore simultaneous movements which the healthy hand can naturally perform are not possible [24]. In this context, to grab a bottle of water, the user has to first rotate the prosthetic hand into the right angle to then open and close it for grasping, instead of the natural hand behavior of rotating and opening simultaneously and then closing it for grasping.

Secondly, pattern recognition does not provide a proportional control which is necessary to control the speed or force of the prosthetic hand in order to perform hand functions with a controlled force or speed level. Instead, additional methods have been developed to add the proportional component to the control scheme [21], [25].

Using again the example of pouring water into a glass from a bottle, pattern recognition can be used to recognize the four necessary activations (hand open, hand close, wrist supination, and wrist pronation) which are mapped to the two DOF 
of the prosthesis (Figure 3b). In contrast to the conventional control scheme, with pattern recognition the same task can be solved using only four steps, by avoiding the mode switching by co-contraction.

Following the concept by Hudgins et al, several classifiers have been later explored for the use in myocontrol, such as Linear Discriminant Analysis [24], [26], [27], Support Vector Machines [27]-[30], Multi-layer Perceptron Neural Networks [26], [27], [31]-[33], Gaussian Mixture Models [32], [34] or k-Nearest Neighbor [35]. In laboratory conditions, offline data analysis, and able-bodied subjects, these algorithms achieve recognition rates well above $95 \%$ for $>10$ motion classes [24], [36]. However, the robustness of these control methods is still very limited due to the fact that academic research often assumes ideal (or stationary) laboratory conditions which are not met in clinical applications. For example, laboratory studies often fix during the tests the electrode and arm positions. Moreover, the subjects in these experiments are in a different psychological condition than the real prosthetic users and are tested on one-single session, which can last up to a few hours. These conditions do not mimic well the repeated use of over several days. For this reason, none of these methods have replaced the conventional control in commercially available hand prostheses [37].

\subsection{Motivation and objective}

The reason for the failed transfer of the academic research knowledge and results into the development of commercial prosthetic products is the misleading and nonrealistic assumption in the work done in academia and the requirements of industry and prosthetic users in real-life situations [37]. While academia is often focused on publications of theoretical results, industry expects the translation of research 
outcomes into reliable and easy-to-use products. The results found in the majority of scientific publications were acquired in ideal laboratory conditions and the validation was done offline. Just few studies have been performed in more realistic scenarios of prosthetic usage and all indicated that in such more realistic conditions there is a substantial worsening of the ideal results usually reported in the scientific literature. Hargrove et al investigated the effects of electrode shifts [38], [39], Fougner et al focused on the influence of arm position changes [40], Amsuss et al investigated the instability of a pattern recognition system over time [41], Ge et al analyzed the influence of subject's mental state changes in pattern recognition based myocontrol [42]. Additionally, only few hardware or virtual implementations have been used to evaluate the performance of the algorithms in real life conditions [43][47].

In order to start closing the gap between academic research and industry, this PhD project intended to merge the efforts of academia and industry towards the development of a new, technologically advanced and feasible control of myoelectric hand prostheses ${ }^{1}$. This PhD project has been funded by the EU IAPP Project $\mathrm{AMYO}^{2}$ and covers important milestones of the project.

The problem description and objective for this PhD project can be summarized as follows:

\footnotetext{
${ }^{1}$ For instance the Otto Bock Michelangelo Hand: http://www.living-with-michelangelo.com

${ }^{2}$ See http://cordis.europa.eu/projects/rcn/95059_en.html for project details
} 
Development and evaluation of a system for simultaneous and proportional control of two degrees of freedom (DOF) in hand prostheses, by extending the results from academic research and adapting them towards a functional, intuitive, and natural control system.

Very recently, regression approaches have been proposed as an alternative avenue towards multifunctional myocontrol, providing simultaneous and proportional activation of multiple DOF. Specifically, work has been done using Artificial Neural Networks (ANN) [15], [48], [49] or Non-negative Matrix Factorization (NMF) algorithms [14]. Similarly to pattern recognition systems, regression methods also require a phase of training but in contrast to the discrete output of pattern recognition systems, regression methods provide a continuous and proportional estimation of the activation for each DOF. In order to work towards a simultaneous and proportional control system, a regression approach is a more promising solution than a classification approach. In general, the regression system can be understood as a transfer function $\vec{F}$, to transform the $k$-dimensional EMG signal $\vec{s}(t) \in R^{k}$ into the $n$-dimensional control signal $\vec{c}(t) \in R^{n}$ :

$$
\vec{c}(t)=\vec{F}(\vec{s}(t))
$$

Considering the goal and requirements of this project and the achievements of the current research towards myocontrol, the regression approach together with the NMF algorithm to extract from the EMG signal low dimensional command signals [14] have been identified as the most promising approach for this PhD project. This control scheme has been presented in a theoretical framework in academia and tested in offline, laboratory conditions with static muscle activation for the estimation of force [14]. However, realistic conditions for the daily use of prosthetic devices 
include dynamic muscle activation, counter-movements and external influences, such as noise [23]. Therefore, substantial improvements and extensions are necessary to translate regression systems into an industrially viable approach for myocontrol. The following three major challenges have been identified:

(1) Conditioning of the EMG signal quality to face more realistic (non-laboratory) conditions.

(2) Design of an evaluation paradigm suitable for online testing.

(3) Development and implementation of a real-time regression system for online estimation, extending from the work of Jiang et al [14];.

These improvements not only make the system applicable in realistic conditions but also make substantial scientific step forwards with respect to the original algorithm, so that a fully new method was generated at the end of this work.

Chapter 2 presents the methodology of this work and contributions to the field. According to the three major challenges given above, the corresponding developments are presented in three subchapters. The evaluation of the developed methods has been done in two studies, which are presented in chapter 3 . The thesis is closed by the discussion in chapter 4 . 


\section{Methodology}

As the quality of the surface EMG signal is the key to achieve a robust and reliable myocontrol system, in chapter 2.1 a newly developed Adaptive Common Average Reference filter [50] is presented, as an important contribution towards noise reduction and increased selectivity of the EMG signal. Besides the theoretical background of this filter, a qualitative validation of the signal is presented to underline the benefits of this algorithm.

Chapter 2.2 introduces a Virtual Evaluation Paradigm and Performance Assessment Task which was designed for the online evaluation and assessment of the performance of the proposed myocontrol algorithms. Hence, besides the virtual control paradigm, performance metrics are also provided.

Finally, chapter 2.3 presents the online myocontrol system developed on the basis of the work by Jiang et al [14] but with substantial modifications, such as the estimation of kinematics instead of force and the possibility of use in full dynamic tasks and in online manner. 


\subsection{Adaptive Common Average Reference Filtering}

The Adaptive Common Average Reference has been developed during the first phase of this PhD project. This filter is an adaptive modification of the classic CAR filter [51], and is used to optimize the selectivity of the EMG signal for different gestures, while simultaneously improving the signal-to-noise ratio of the acquired signal.

This subchapter is based on a submitted manuscript [50], therefore text or results taken from this manuscript are not cited explicitly.

\subsubsection{Motivation}

In myocontrol applications, the quality of the EMG signal is one of the most important factors for performance. Both for classification and regression approaches, the noise level and the spatial selectivity of the input EMG signal substantially impact the performance. Therefore, extensive work has been devoted in the past to increase the quality and information content of the control signal [52]-[54].

Previous pre-processing methods for EMG have focused on the reduction of common noise and of crosstalk. These methods include spatial filtering, that can be performed with bipolar, double-differential [55]-[57], Laplacian or other twodimensional configurations [58], [59]. Spatial filters for EMG have usually a high-pass spatial transfer function that eliminates the spatial DC and reduces the influence of distant sources (see [58], [59] for details). Additionally, time-domain filters are regularly used to eliminate the power line interference [60] or to reduce motion [61] and ECG artifacts [62]. 
For multi electrode systems, the common average reference (CAR) filtering has also been used in EMG applications [61], [63], although this approach is most often implemented in EEG recordings [51], [64]. The CAR filtering is based on a sampleby-sample subtraction from each channel of the average signal value over all channels. As other spatial filters for EMG processing, CAR has a high-pass transfer function that eliminates the spatial DC.

In EEG analysis, the CAR filter is used to remove dominant noise components present in all channels and thus enhances the signal-to-noise ratio (SNR) by rejecting the spatial DC component. For EEG signals, the common noise has usually greater power than the signal component of interest, thus the CAR filter has a strong effect on improving the SNR [51]. However, when the CAR is applied to EMG signals, it may actually introduce undesired components. In contrast to EEG, the common noise in EMG recordings is indeed usually smaller than the signal component. Thus, the virtual reference of the CAR is not dominated by the common noise components, but additionally contains the inverted signal of the channels with
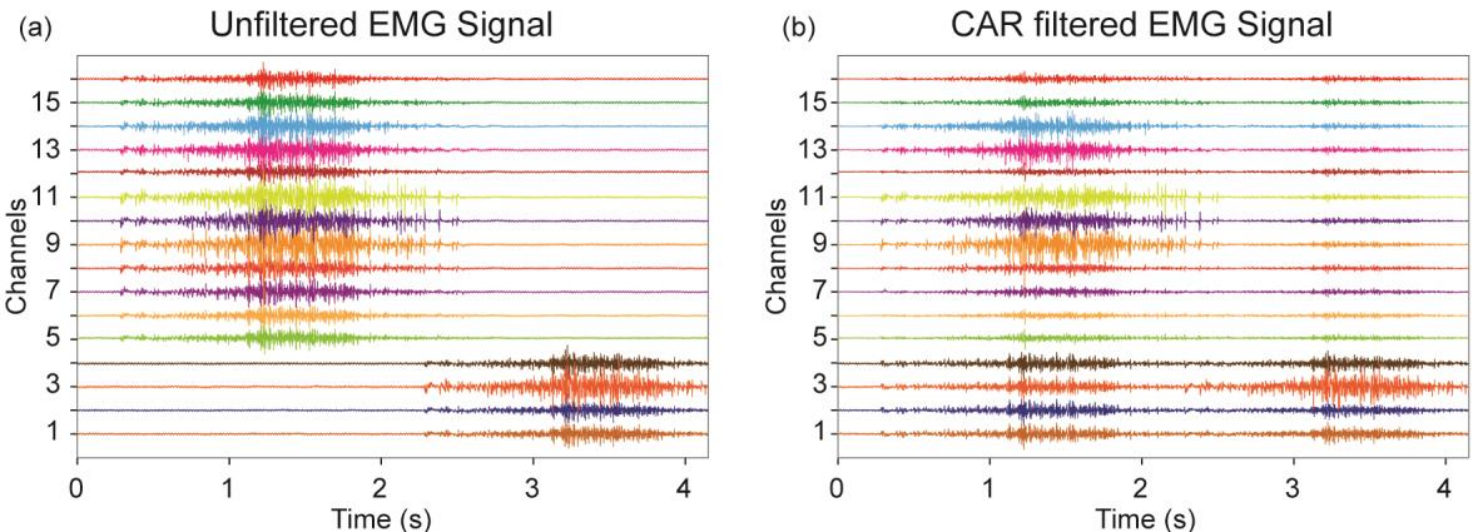

Figure 5: (a) Raw multi-channel EMG signal (16 monopolar channels). (b) The same signal filtered using the classic common average reference (CAR) method. For the first EMG burst (approximately from $1 \mathrm{~s}$ to $2 \mathrm{~s}$ of the recording) signal components are added by CAR in channels 1-4, which are originally not active. A similar effect is visible for the second burst of activity for channels 5-16. 
large EMG activity.

The effect of the CAR filter on EMG may thus be even a reduction in spatial selectivity (see Figure 5 for an example), which is usually contra productive for myocontrol applications.

In this chapter, an extension of the CAR approach is presented based on an adaptive calculation of the common mean value on a subset of EMG channels. As mentioned before, this method will be introduced as adaptive common average reference (ACAR). Finally, the effectiveness of the proposed ACAR will be tested in a quantitative analysis and demonstrated on myocontrol applications.

\subsubsection{Methods}

\subsubsection{Adaptive common average reference (ACAR)}

The ACAR algorithm is built on the assumption that the signal intensity on all channels is greater than the common noise. This is generally the case for EMG recordings, as shown in Figure 5(a). Additionally, we make the assumption that the signal power of more distant sources is smaller than that of closer sources. Using these hypotheses, ACAR is based on the calculation of the common mean from only a subset of channels, contrary to the classic CAR that computes the mean over all channels. By selecting this subset in a manner that the channels with EMG activity (and therefore information content) are excluded, the resulting mean reference signal is dominated by the common noise, which needs to be eliminated.

Considering a signal $S \in R^{K \times N}$ with $K$ channels and $N$ samples, the signal is separated in non-overlapping windows 


$$
W_{j}=\left[\begin{array}{ccc}
w_{1,1}^{j} & \cdots & w_{1, l}^{j} \\
\vdots & \ddots & \vdots \\
w_{k, 1}^{j} & \cdots & w_{k, l}^{j}
\end{array}\right] \in R^{K \times L}
$$

of length $L$, where $j$ denotes the order of the windows. For each window, we compute the channel-wise signal intensity

$$
\begin{gathered}
\vec{p}_{j}=\left[p_{1 j}, p_{2 j}, \ldots, p_{K j}\right]^{T} \text {, where } \\
p_{k j}=\sum_{l=1}^{L}\left|w_{k, l}^{j}\right|,
\end{gathered}
$$

is the sum over absolute values (average rectified value). Based on $\vec{p}_{j}$, a subset of channels $\Lambda_{j} \subseteq\{1,2, \ldots, K\}$ with the lowest signal intensity is selected. For this channel selection, several approaches can be used. A simple method is to select the ${ }^{\lceil K / 2\rceil}$ channels with the lowest intensity (further denoted as $\mathrm{K} / 2$ criteria). Alternatively, it is possible to set a threshold on the intensity estimate, either dynamic (i.e., mean or median across the channels) or with a fixed value. In any case, $\Lambda$ should always contain more than one channel, to avoid susceptibility towards a single channel. For the dynamic threshold this implies a second override step, to use the three channels with lowest intensity if the dynamic threshold results in less than three channels for $\Lambda_{j}$. Finally, the sample-wise mean value is calculated for the selected channel subset as:

$$
\begin{gathered}
\vec{\mu}_{j}=\left[\mu_{j, 1}, \mu_{j, 2}, \ldots, \mu_{j, L}\right], \text { where } \\
\mu_{j, l}=\sum_{k \in \Lambda_{j}} w_{k, l}^{j}
\end{gathered}
$$


and subtracted from all channels in $W_{j}$

$$
W_{j}^{*}=W_{j}-\left[\begin{array}{c}
\vec{\mu}_{j} \\
\vdots \\
\vec{\mu}_{j}
\end{array}\right]_{k \times 1} .
$$

The filtered signal $S^{*}=\left[W_{j}^{*}, W_{j}^{*}, \ldots, W_{j}^{*}\right]$ can then be obtained by concatenating all processed windows. The resulting filter is a spatial filter changing its transfer function over time.

\subsubsection{Quantitative evaluation of noise reduction and selectivity}

To quantify the common noise reduction and the increase in signal selectivity, the proposed filter has been applied to EMG data recorded with $2048 \mathrm{~Hz}$ sampling rate from 16 monopolar electrodes, placed as pairs equidistantly in a circular manner around the proximal third of the forearm [65]. All measures have been performed under the approval of the local ethics committee. Out of the 16 electrodes, two were located above the flexor and two above the extensor muscles of the wrist. The metrics used were chosen according to [58], where wrist flexor and extensor muscles were used as agonist/antagonist pair. As the ACAR filter is designed for the use in myoelectric applications, the subjects contracted their muscles voluntarily up to MVC instead of using stimulation, as was done in [58]. Based on the acquired data for maximal flexion and extension, the two following signal-to-noise ratio (SNR) indexes were calculated as performance metrics.

To assess the within-channel SNR, the data of the same channel were used to calculate the signal power for the relaxation phase $P_{\text {agonistrelax }}$ and for full contraction $P_{\text {agonistMvC }}$, leading to the factor: 


$$
S N R_{\text {within }}=\frac{P_{\text {agonist,MVC }}}{P_{\text {agonistrelax }}}
$$

The between-channel SNR was calculated using the data of one agonist and one antagonist channel for the same contraction:

$$
S N R_{\text {between }}=\frac{P_{\text {agonist,MVC }}}{P_{\text {antagonist } \mathrm{MVC}}}
$$

For both cases, a greater SNR represents a better signal quality for the agonist channel. The calculation was done for 200-ms windows, each subject performing four contractions for both wrist flexion and wrist extension. This resulted for each method in two pairs of $S N R_{\text {within }}$ and $S N R_{\text {between }}$, one for wrist flexion and one for wrist extension.

Based on the above indices, the ACAR filter was tested using different channel selection criteria. The criterion that resulted in best performance was used for comparing ACAR with the unfiltered raw data, bipolar filtered data, and the data filtered by the classic CAR.

The results were statistically tested using two-factor ANOVAs, with the pre-processing method and the subject as factors. A significance level of $\alpha=0.05$ was used for all tests.

\subsubsection{Impact on myocontrol based on pattern classification}

The performance of the ACAR was also evaluated on classical pattern classification methods for myocontrol, to directly prove the impact of the new filter in myocontrol. For this purpose, four TD features (RMS, wave length, zero crossing, slope sign changes, [20]) extracted from the 16 channels (window length of 400 samples) were 
obtained from the unfiltered EMG and the EMG filtered with CAR and ACAR, using the same electrode setup as above. The data were recorded from seven able-bodied subjects (2 female, 5 male, age range 25-57) during six wrist movements (flexion, extension, abduction, adduction, supination, and pronation), each performed 4 times for 4 seconds at $50 \%$ MVC force. For quantitative comparison of the approaches, the separability index (SI, larger indicates better performance) and the mean semiprincipal axes (MSA, lower indicates better performance) [66] were used. Additionally, the quotient of SI and MSA (distinctness coefficient DCOFF = SI/MSA, larger indicates better performance) was introduced as a further performance metrics.

\subsubsection{Impact on regression based myocontrol systems}

Finally, the impact of the ACAR filter on the regression myocontrol system developed during this PhD project has also been evaluated. This analysis has been highly relevant for this thesis and is therefore presented separately in chapter 3.1 .

\subsubsection{Results}

\subsubsection{Quantification of noise reduction and selectivity}

The results of the impact of the channel selection criteria are shown in Figure 6 for a
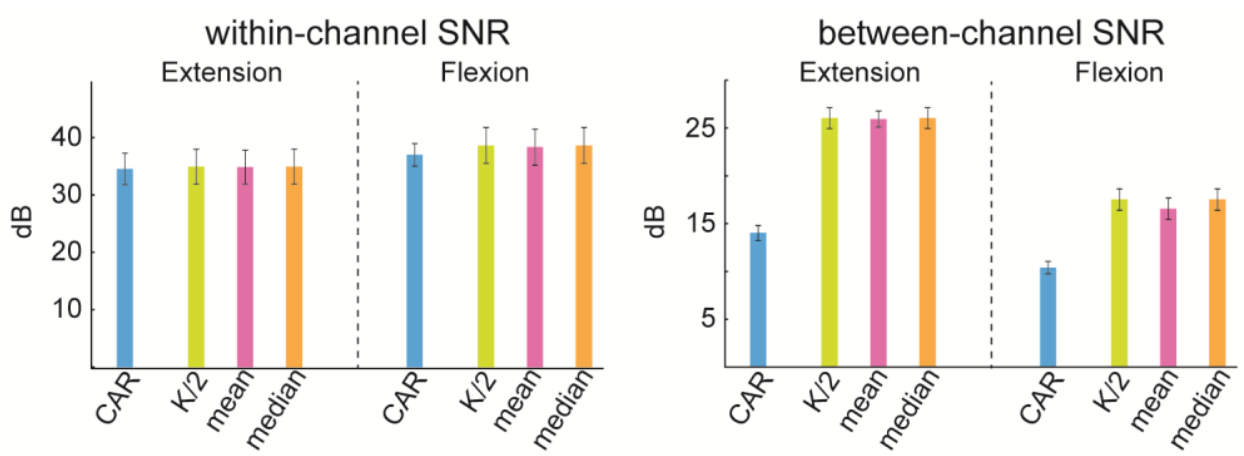

Figure 6: Comparison of the different selection criteria for the ACAR algorithm. These results are obtained from subject 3 and show that all methods exceed the SNRs of the standard CAR. The same results were found for the other subjects. 

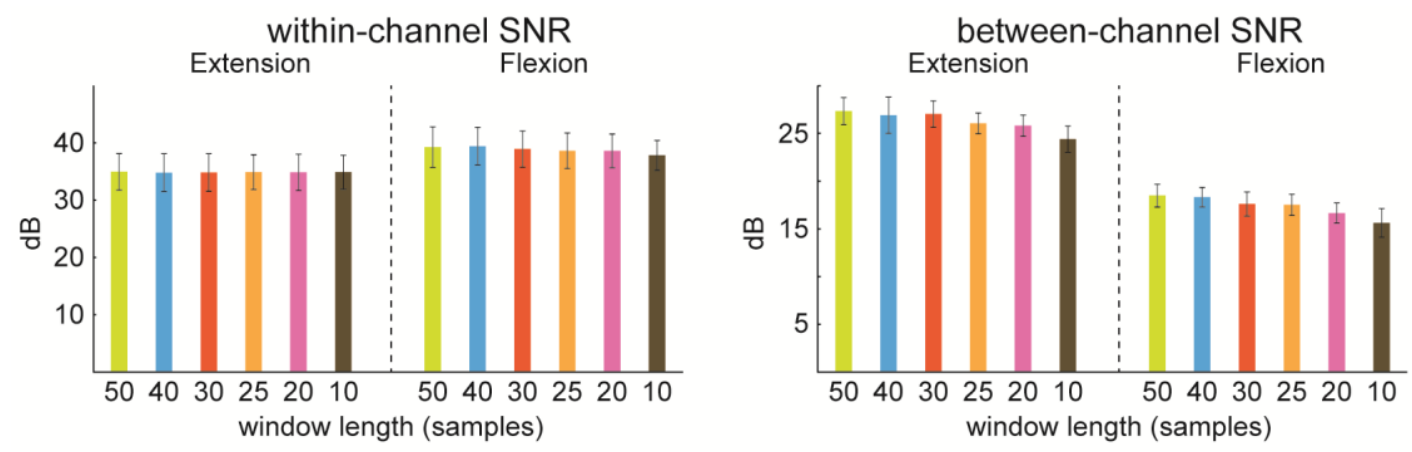

Figure 7: Comparison of the SNRs using $K / 2$ criteria and different window length $L$ for subject 3. The results show that a longer window length has a stronger impact to the between-channel SNR, therefore increasing the separability. Similar results were found for the other selection criteria and subjects.

representative subject. Besides the CAR and ACAR with $\mathrm{K} / 2$ method, also the mean and the median threshold within each 25 sample window was tested (for definition of the parameters see chapter 2.1.2.1). Both $\mathrm{K} / 2$ and the median criteria yielded equally good results, which were confirmed also for the other subjects.

Additionally, the impact of the window length $L$ for the $\mathrm{K} / 2$ selection criteria is shown for the same subject in Figure 7. The K/2 method was used in this case due to its simplicity and similar performance to other selection methods (Figure 6). The analysis for the other subjects showed very similar results. The selection method and the window length did not impact the performance when analyzed statistically over all subjects (all $p>0.05$ ). Therefore, the $\mathrm{K} / 2$ method with 25 -samples window length was used in all subsequent analyses.

The comparison among methods for common noise reduction and signal selectivity is shown in Figure 8. Over all subjects, both the within-channel SNR and the between-channel SNR were the highest for the ACAR method (K/2 method, 25 samples) for both wrist flexion and extension. For the between-channel SNR, which represents the channel selectivity, the ACAR exceeded the CAR by a minimum of $6 \mathrm{~dB}$ for all subjects. 


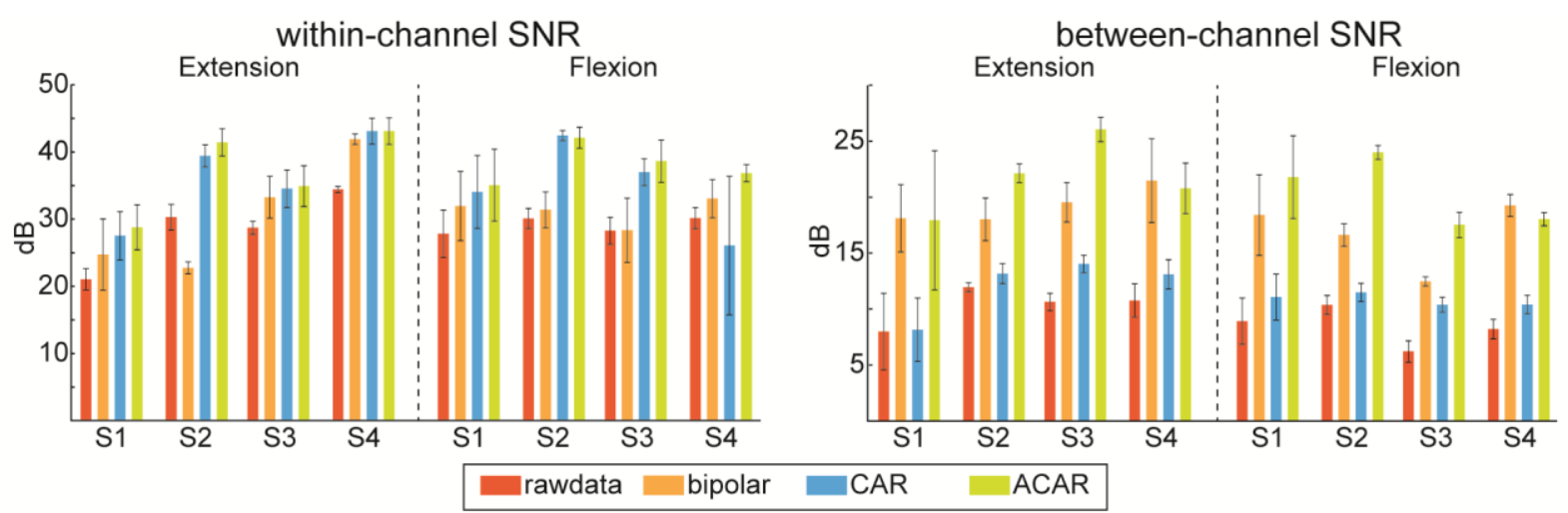

Figure 8: The results of the offline analysis for the four preprocessing methods are shown grouped by the four subjects (S1-4). For the ACAR the window length chosen was 25 samples with the K/2 channel selection criteria. The bars indicate the mean values over the four measures for each subject, with the standard deviation shown on top.

The statistical analysis for the comparison resulted in a strong significance for all four metrics (all $p<<0.05$ ).

\subsubsection{Myocontrol based on pattern classification}

The feature spaces for a representative subject calculated from the unfiltered EMG signal as well as the signal filtered with CAR and ACAR are presented in Figure 9, where, for graphical representation, only the first two principal components of the feature space are shown. The clusters for each wrist movement are represented by the different colors.

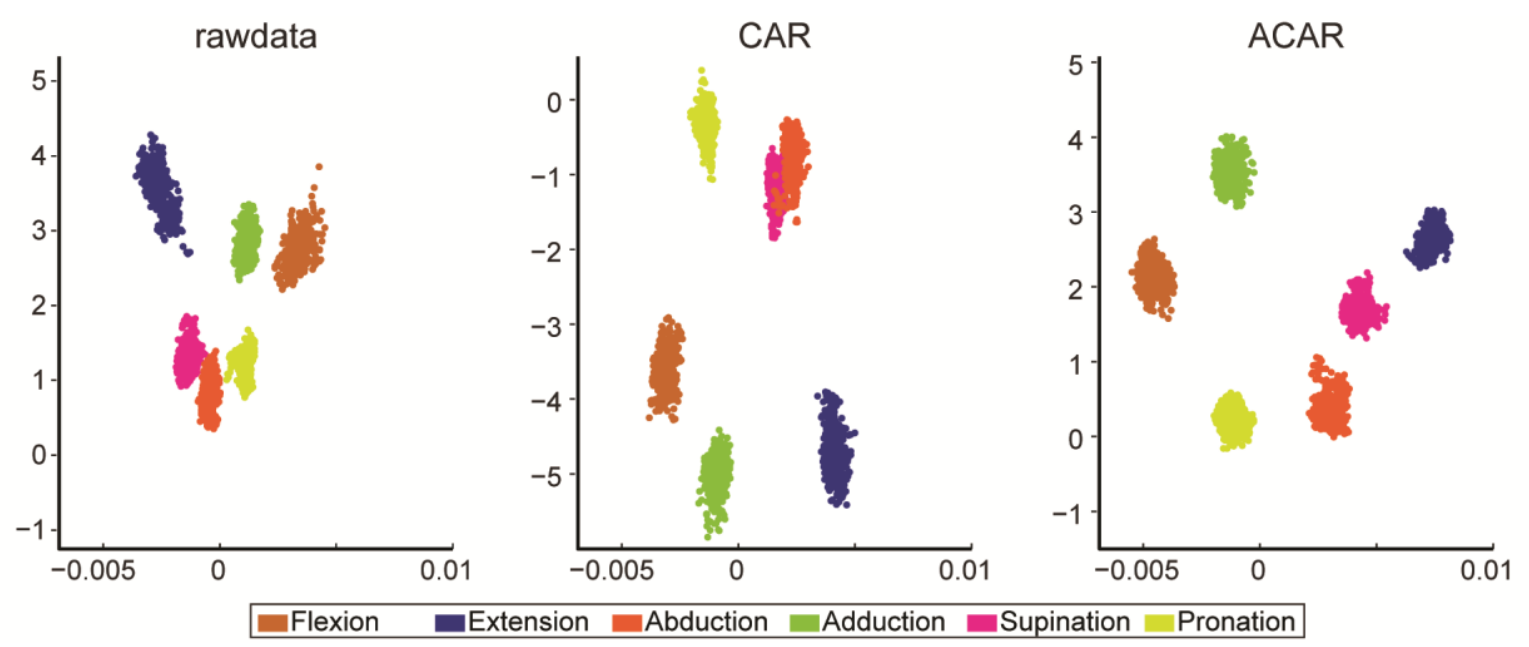

Figure 9: Projection of the LDA spaces for subject 3 onto the first two components, separated by the three input signals. Only the unfiltered monopolar signal and the CAR and ACAR filtered data was used. (Note: the three plots have the same axis scaling) 


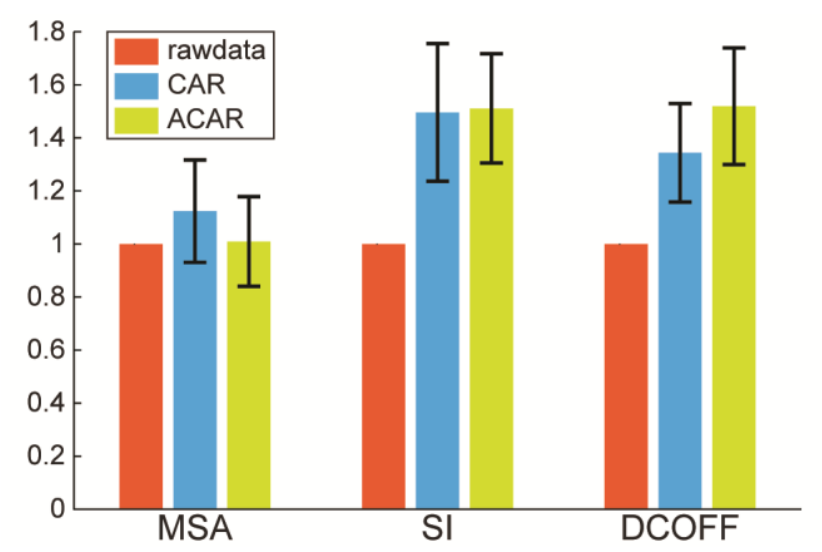

Figure 10: The three performance metrics (MSA, SI, DCOFF) have been calculated for all subjects and the three different preprocessing methods. The figure depicts the mean values and standard deviations for the four subjects. For better comparison, the values have been normalized on subject base using the results for unfiltered data (orange) as reference.

For all subjects the three metrics (MSA, SI, DCOFF) were calculated and are presented in Figure 10. Generally, the MSA values, as a measure of cluster density, were the lowest either for the unfiltered data or ACAR, while the highest SI was obtained by ACAR or CAR. Finally, the DCOFF as a combination of SI and MSA always yielded the highest values for the ACAR preprocessing.

\subsubsection{Discussion}

A new time-varying spatial filter for myocontrol applications has been proposed. The filter is based on the subtraction from each channel of the mean value of the subset of channels with minimal signal intensity.

The tests on the channel selection criteria for the proposed ACAR filter identified the $\mathrm{K} / 2$ criterion as adequate and simple. The median and mean criteria require an additional thresholding without significant improvement. Regarding the window length, 25 samples (equals $12.2 \mathrm{~ms}$ ) were identified as a good trade-off for SNR improvement and response time of the filter. An increased window length might give a slightly better performance in terms of signal quality, but would be less appropriate for quick control changes. Using this configuration set, the ACAR filter has been 
shown superior for gain in signal quality to all other tested filters. These included also the bipolar recording, which is the current state of the art in industrial applications.

Furthermore, we have also shown a gain in class separability when using the ACAR filter on the feature space for pattern classification. As stated by [66], an increase in $\mathrm{SI}$, as identified for the CAR and ACAR, reveals more distinct classes in the transformed feature space, resulting either from more dense clusters or greater distances between classes. For the presented results for SI, the CAR and ACAR can be used to increase the separability of the contractions in comparison to unfiltered data. Additionally, the MSA is reduced for all subjects when using the ACAR in comparison to CAR. This indicates that the clusters are both more compact and better distributed in the feature space with ACAR filtering. In comparison to the unfiltered data, the MSA was slightly increased, but an increasing MSA can be compensated by a proportionally increase in SI, to ensure separability between the classes. These combined effects of SI and MSA can be expressed by the DCOFF, according to which the ACAR outperformed the other methods.

\subsubsection{Conclusion}

In this chapter the ACAR filter has been presented as an adaptive approach to improve the signal quality of monopolar recordings, by significantly reducing the common noise level and increasing the spatial selectivity. This improvement has been quantified using an SNR measure and comparing it to three other methods commonly used in myocontrol. In contrast to other preprocessing methods, the ACAR filter does not need training or adjustments, and is based on simple calculations suitable for online applications. The impact of the increased signal quality and separability has then been presented for classical EMG pattern 
classification, where the ACAR pre-processing generated an increased separability and distinctness of the different gesture classes in the feature space.

Finally, as shown in the independent study presented in chapter 3.1 , the ACAR is a key component for this $\mathrm{PhD}$ project towards an online regression control system, which significantly improves the controllability. 


\subsection{Virtual Evaluation Paradigm}

As pointed out in chapter 1 , most scientific work in myocontrol has been focused on the evaluation of the acquired data in an offline manner (i.e. [20], [23], [54], [67][69]). However, to assess the performance of myocontrol algorithms in daily life situations and include the adaptive behavior of the user [70], an online implementation and evaluation are needed. This approach provides the user with a (visual) feedback plus the possibility to react to it.

An obvious solution is to implement the control algorithm to be tested into a real prosthetic hand, to perform daily tasks, like pouring a glass of water or manipulating objects. Unfortunately, this solution requires the availability of the hardware and the final fitting of the prosthesis to each user. Additionally, it is difficult to measure performance metrics for daily tasks. Standardized methods like the Southampton Hand Assessment Procedure (SHAP) [71] or the Action Research Arm Test (ARAT) [72], [73] have been used in clinics and research [74], especially to assess the impairment of patients. However, those tests only allow outcome oriented metrics, for instance the time to finish a task. These measures do not provide information on the smoothness of the control or the efficiency of the task completion.

An alternative approach for pre-testing before prosthetic fitting consists in using a virtual hand prosthesis embedded in a 3D virtual and immersive environment. However, the depth perception is limited and a virtual 3D environment is not suitable for all subjects [75]-[78].

In order to provide a simple but intuitive assessment of the controllability for two DOF, a Virtual Evaluation Paradigm (VEP) has been developed within this project and implemented using a real-time equivalent software environment [79]. The VEP is 
designed to be used on different screens and monitor devices, therefore the units for positions and distances in the VEP are density-independent pixels ( $\mathrm{dp}$ ) [80].

\subsubsection{Subject interface}

The VEP is based on a subject interface, representing two main DOF (DOF 1: wrist flexion/extension, DOF2: wrist pronation/supination) to the subject (Figure 11). This representation is defined by an arrow of length $2 \hat{y}, \hat{y}=60 \mathrm{dp}$, that is positioned in the center of the target area as the neutral position. The flexion and extension of the wrist is mapped proportionally to the arrow's position on the horizontal axis, using the control signal $v(t)$. In case of a right-handed subject, the horizontal displacement of the arrow to the right side $(v(t)>0)$ corresponds to the extension angle of the wrist (see Figure 12), while the wrist flexion is represented by negative values for $v(t)$. The maximum flexion and extension angle is indicated by the markers on the horizontal axis in the subject interface.
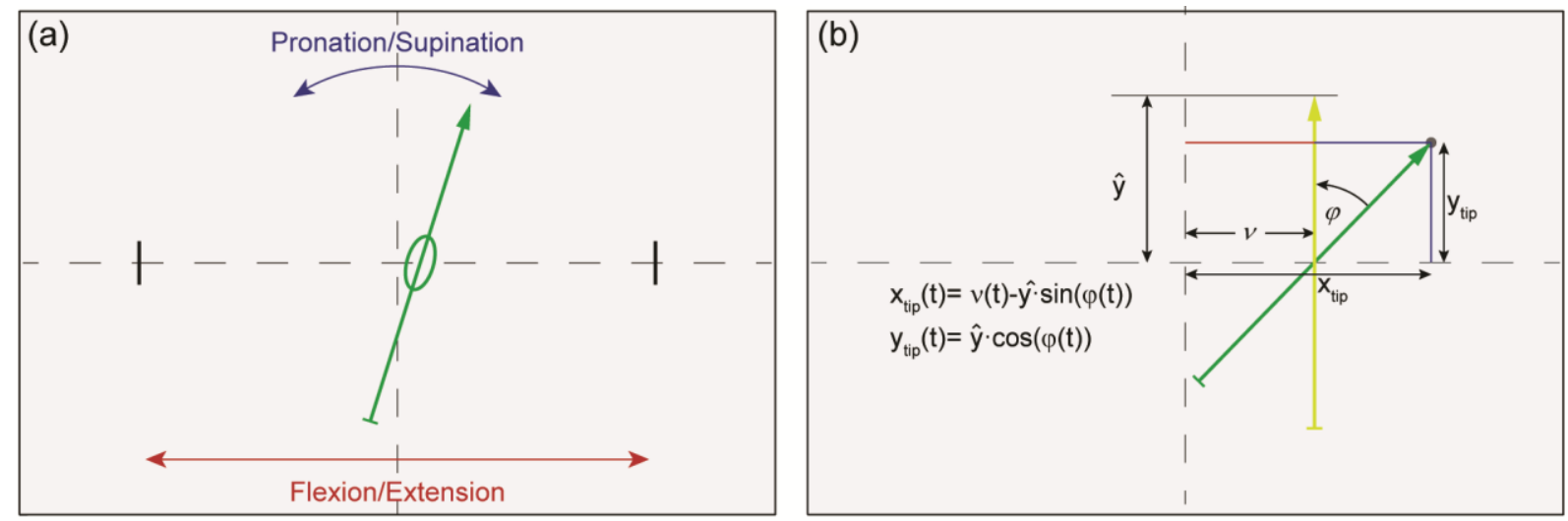

Figure 11: (a)The user interface of the Virtual Evaluation Paradigm (VEP) developed for online evaluation. The flexion and extension of the wrist is represented through the horizontal position of the arrow (green), the wrist rotation by the rotation of the arrow. The vertical markers indicate maximum flexion and extension. The rotation is limited by $+/-90^{\circ}$. (b) The position of the arrow's tip $\left(x_{\text {tip }}(t), y_{\text {tip }}(t)\right)$ can be calculated from the two control signals $(v(t)$ $, \varphi(t))$, using the equations provided. 


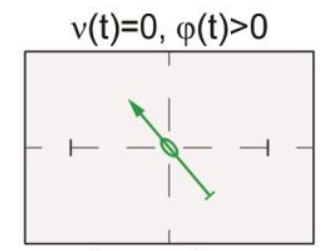

Pronation

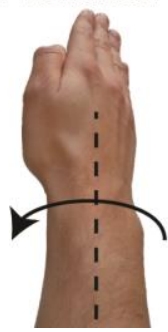

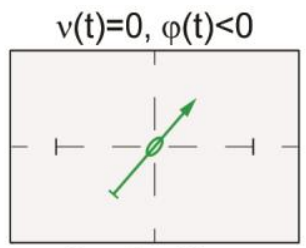

Supination

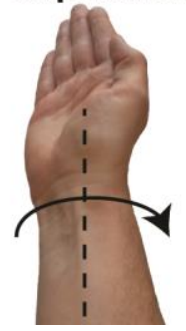

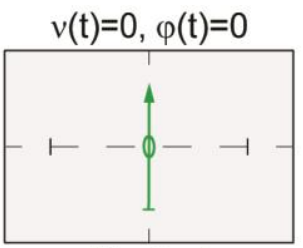

Neutral

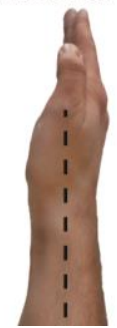

$v(\mathrm{t})<0, \varphi(\mathrm{t})=0$

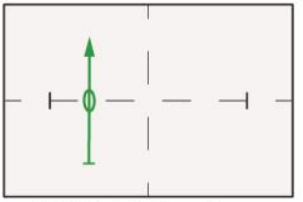

Wrist Flexion

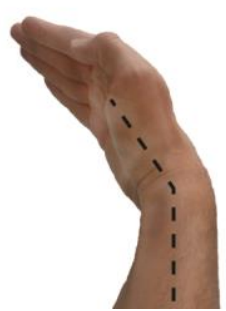

$v(t)>0, \varphi(t)=0$

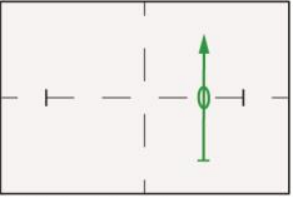

Wrist Extension

Figure 12: Mapping of the arrow to the hand gestures for single DOF movements. This figure represents the subject's view when looking at the screen, with the hand pointed forward. The example is given for a right handed subject. For left handed subjects pronation and supination are swapped, as well as wrist flexion and extension.

Similarly, the rotation of the arrow represents the rotation of the wrist (pronation/supination), encoded by the control signal $\varphi(t)$ (Figure 11b). The rotation is limited by $+/-90^{\circ}$ rotation of the arrow from the upright position (see Figure 12).

In case the subject is left-handed, the interface can be used in the same manner, by switching flexion with extension and pronation with supination.

Extending from the two control signals provided, the position of the arrow's tip $s_{\text {tip }}(t)=\left[\begin{array}{c}x_{\text {tip }}(t) \\ y_{\text {tip }}(t)\end{array}\right]$ can be calculated using the equations given in Figure $11 \mathrm{~b}$.

For a consistent user experience, the VEP can be used both to acquire labeled data for training and calibration of control algorithms and to evaluate the resulting control performance.

\subsubsection{Guided calibration mode}

In the guided calibration mode, the VEP is used to acquire labeled EMG data for the wrist movements, by providing a prompt to the subject. This setup is presented in Figure 13. Specifically, the generated prompt consists of the two control signals $v(t)$ 


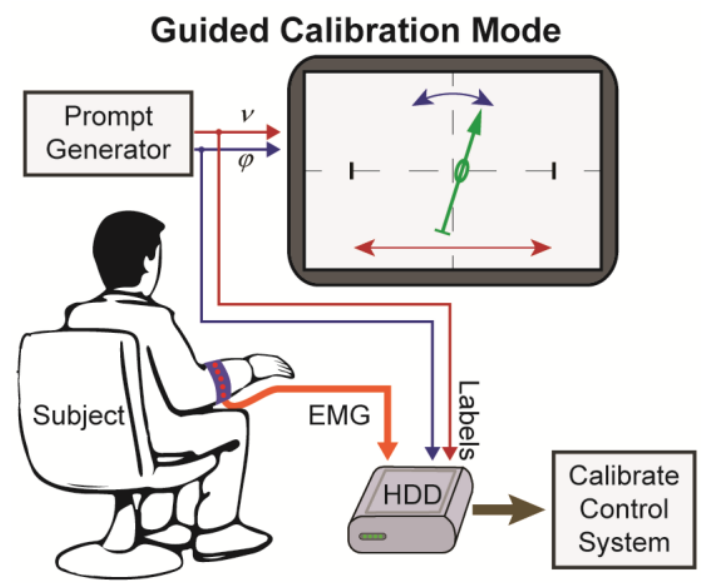

Figure 13: During the Guided Calibration Mode the VEP is controlled by an external prompt generator. The subject follows this cue and the EMG is recorded synchronously with the presented prompt as labels. This labeled data can be used to calibrate the control system.

and $\varphi(t)$, which are sent to the VEP to control the arrow position and to provide a guidance to the user and the recording system, to be recorded synchronously with the EMG data.

\subsubsection{Free calibration mode}

Similar to the guided calibration mode, the free calibration mode is used to acquire labeled EMG data for wrist movements. Instead of providing a prompt to the subject, the actual wrist and hand kinematics are recorded using an XSens MTx Motion Capture System ${ }^{3}$, simultaneously with the EMG signal (see Figure 14). Hence, the subject can freely perform different movements for which labels are provided by the motion capture system.

For the recording of the kinematics, in total three XSens MTx sensors are placed on the subject's arm, as shown in the left part of Figure 14. The first sensor (MT1) is placed on the back of the hand (dorsal) with the sensor's x-axis pointing towards the thumb. The second sensor (MT2) is placed dorsally on the distal part of the forearm, right before the wrist joint, with the $x$-axis pointing in distal direction. The third sensor

\footnotetext{
${ }^{3}$ http://www.xsens.com/en/general/xbus-kit
} 


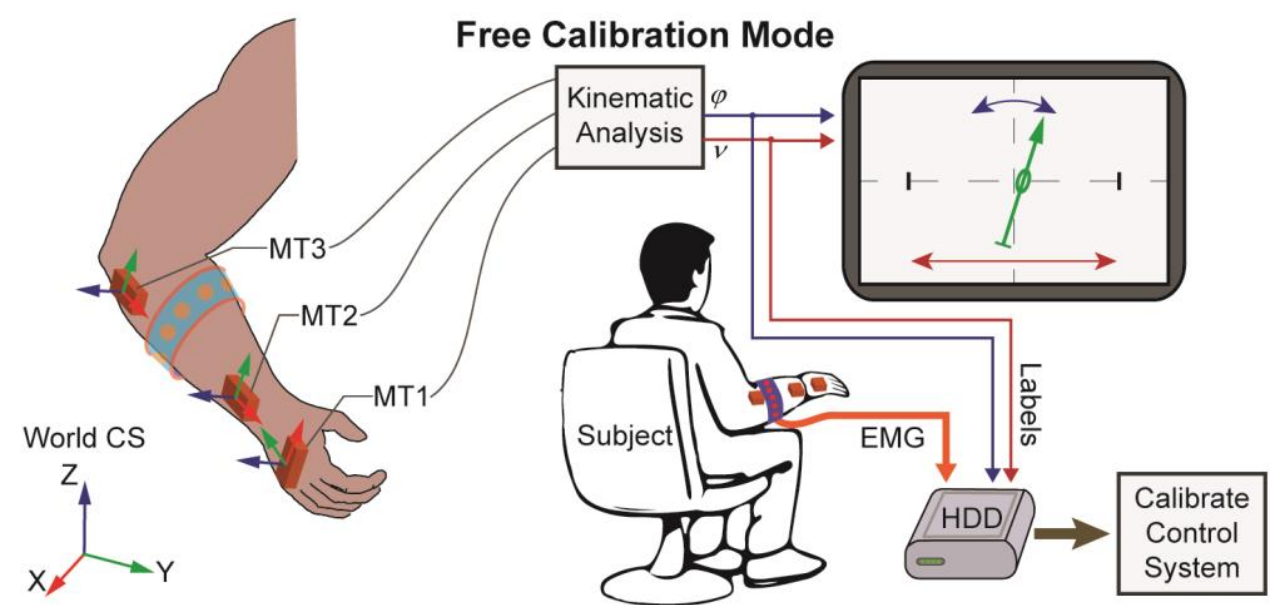

Figure 14: The Free Calibration Mode allows the subject to perform voluntary movements. Using three XSens measurement units the wrist angles are calculated and recorded synchronously with the EMG data.

(MT3) is placed similar as the second one, but shifted to the proximal end of the forearm.

While the placements of the kinematic sensors for intact-limb subjects can be done on the same arm as the EMG electrodes, this is not possible for subjects with limb deficiencies due to the missing limb. To solve this problem for subjects with unilateral conditions, mirrored bilateral training can be used as a training strategy [49]. In this setup, the XSens sensors are placed on the intact-limb side, while the EMG electrodes are located on the contralateral, affected side. Then, during the calibration phase, the subject is asked to simultaneously generate mirrored movements on both sides, thereby generating the muscle activation patterns on the affected side and the corresponding kinematics on the contralateral side.

During the recording, the spatial orientation of the three sensors in relation to the earth magnetic field is measured with $100 \mathrm{~Hz}$ sampling rate. The orientation is represented in the motion capture system through quaternions [81]. From these quaternions, the angles for wrist flexion/extension and rotation are extracted as follows: 
The wrist flexion/extension angle $v(t)$ is calculated using the orientation $q^{\mathrm{MT1}}(t)=\left[w^{\mathrm{MT1}}(t), x^{\mathrm{MT1}}(t), y^{\mathrm{MT1}}(t), z^{\mathrm{MT1}}(t)\right]$ of the sensor MT1 and the orientation $q^{\mathrm{MT2}}(t)=\left[w^{\mathrm{MT2}}(t), x^{\mathrm{MT} 2}(t), y^{\mathrm{MT2}}(t), z^{\mathrm{MT2}}(t)\right]$ of sensor MT2. More specifically, the vector $w_{\mathrm{MT1}}=[0,-1,0]_{\mathrm{MT1}}^{T}$ (negative $\mathrm{y}$-axis) in the coordinate system (CS) of MT1 is transformed into the CS of MT2. This is done by calculating the transformation matrix $T_{\mathrm{MT1}}^{\text {world }}\left(q^{\mathrm{MT1}}\right)$ from the CS of MT1 to the world CS, and the transformation matrix $T_{\text {world }}^{\text {MT2 }}\left(q^{\mathrm{MT2}}\right)$ from the world CS to the CS of MT2. Finally, the angle $v(t)$ can be obtained as the angle between the transformed vector $w_{\mathrm{MT} 2}=T_{\mathrm{worl}}^{\mathrm{MT2}}\left(q^{\mathrm{MT2}}\right) \times T_{\mathrm{MT} 1}^{\text {world }}\left(q^{\mathrm{MT} 1}\right) \times w_{\mathrm{MT} 1}$ and the $\mathrm{x}$-axis of MT2 $x_{\mathrm{MT} 2}=[1,0,0]_{\mathrm{MT} 2}^{T}$, both projected into the XZ-plane of MT2.

In a similar way, the wrist rotation angle $\varphi(t)$ is calculated from the orientation of MT2 $\left(q^{\text {MT2 }}\right)$ and MT3 $\left(q^{\text {MT3 }}\right)$. In this calculation, $\varphi(t)$ is equal to the angle between the z-axes of MT2 projected into the CS of MT3 $\left(a_{\mathrm{MT} 3}=T_{\mathrm{world}}^{\mathrm{MT} 2} \times T_{\mathrm{MT} 1}^{\mathrm{world}} \times a_{\mathrm{MT} 1}\right.$, $\left.a_{\mathrm{MT} 2}=[0,0,1]_{\mathrm{MT} 2}^{T}\right)$ and the z-axis of MT3 $z_{\mathrm{MT} 3}=[0,0,1]_{\mathrm{MT} 3}^{T}$, both projected into $\mathrm{YZ}$ plane of MT3.

Note: While the Free calibration mode has been used in the early stage of the PhD project [65], [79], the results presented in chapter 3.2 and the most recent publications [82], [83] where acquired using the Guided calibration mode. However, as project partners [84] and collaborators [85] continue using the developed system, it has been included as a part of this thesis.

\subsubsection{Evaluation mode}

During the evaluation phase, the calibrated control system is used to extract control signals from the EMG data, recorded online from the subject's forearm. Due to the 
flexibility of the VEP, algorithms based on pattern recognition, regression algorithms or any other myocontrol method can be used [83]. The only requirement is that the control algorithm provides the two continuous control signals $v(t)$ and $\varphi(t)$ as output.

The extracted control signals are send to the VEP, controlling the arrow's position, as illustrated in Figure 15.

\subsubsection{Performance assessment task}

With the intention of assessing the online performance of a myocontrol algorithm, the VEP in Evaluation Mode has been extended by a Performance Assessment Task. As for this purpose the arrow position is controlled by the estimation algorithm, the Performance Assessment Task is part of the evaluation mode.

The task itself is designed as a target hitting task. Therefore, different targets in a circular shape are placed within the area of reach of the tip of the arrow. Generally, three types of targets can be used. Targets of type 1 are placed on a horizontal line, vertically shifted up by half the arrow length (Figure 16, magenta targets). To reach these targets, only the first DOF (wrist flexion/extension) has to be used.

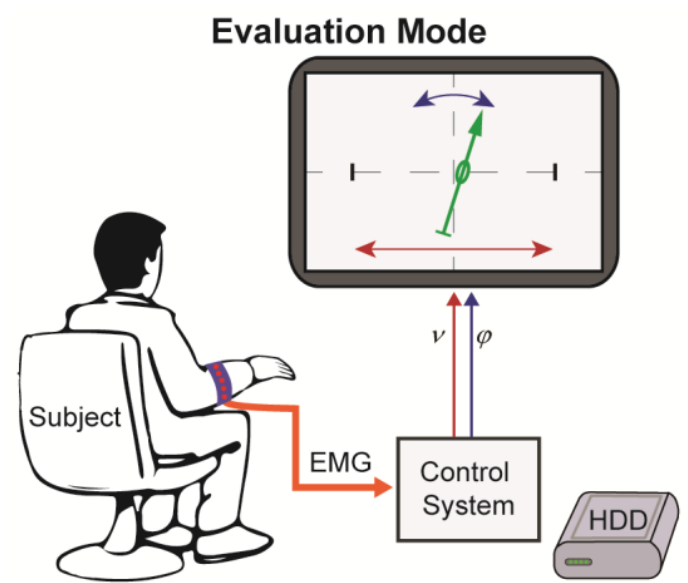

Figure 15: In the Evaluation Mode the output of the calibrated control system is used to control the arrow in VEP. 


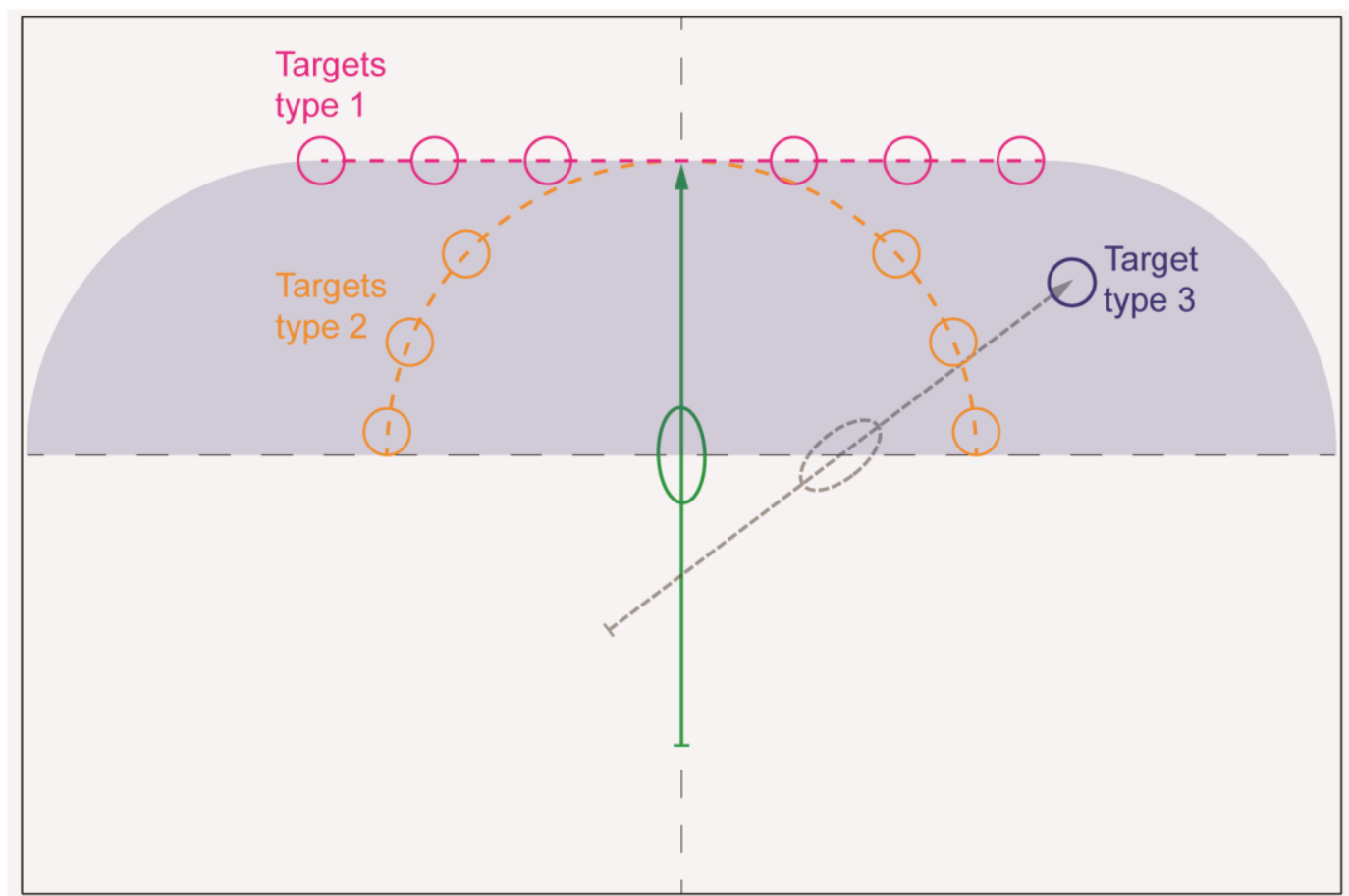

Figure 16: Examples for the targets in the Performance Assessment Task. The target types are presented in different colors. The magenta targets can be reached using solely wrist flexion/extension. The orange targets only require wrist pronation/supination. Finally, the blue area indicates the area that can be reached using both DOF simultaneously. An example for these combined targets is presented in blue.

Correspondingly, the targets of type 2 are placed on a circle with the radius equal to half the arrow length (Figure 16, orange targets), requiring the subject to use only DOF 2 (wrist pronation/supination) to reach the target. Finally, the targets of type 3 are placed within the whole area that can be reached by the top of the target, requiring the user to control both DOF simultaneously. In Figure 16 this area is indicated by the blue colored shape and an exemplary target displayed as a blue circle.

A task is defined by a single target circle colored in magenta and of diameter $d_{\text {circle }}$ (measured in dp), which is presented to the subject. Starting from the arrow in the center position, the subject is asked after an acoustic start signal to drive the tip of the arrow into the target circle, using the myocontrol algorithm provided. To exclude the case of hitting the target only by chance, the arrow tip has to remain in the target 
for a given dwelling time, specified by the task parameter $T_{\text {dwell }}$. If the subject is able to reach and remain in the target, the task is considered completed. To give a feedback to the subject during the dwelling time, the circle's color is changed to yellow while the arrow tip is in the target. A successful task completion is indicated to the subject by another change of the circle color into green and the playback of a rewarding sound. If the subject does not remain in the target for the dwelling time, the color is changed back to magenta. And if the subject is not able to reach and remain in the target within the given timeout $T_{\max }$, the task is considered as failed. A failed task is indicated to the subject by a red circle and a buzzing sound.

To complete targets of type 1 and type 2, the subject should solely use the corresponding DOF, and the other control DOF adversely affects the successful completion of the task. Considering, for instance, targets of type 1 on the horizontal axis, an additional rotation of the arrow makes it impossible for the subject to reach the target.

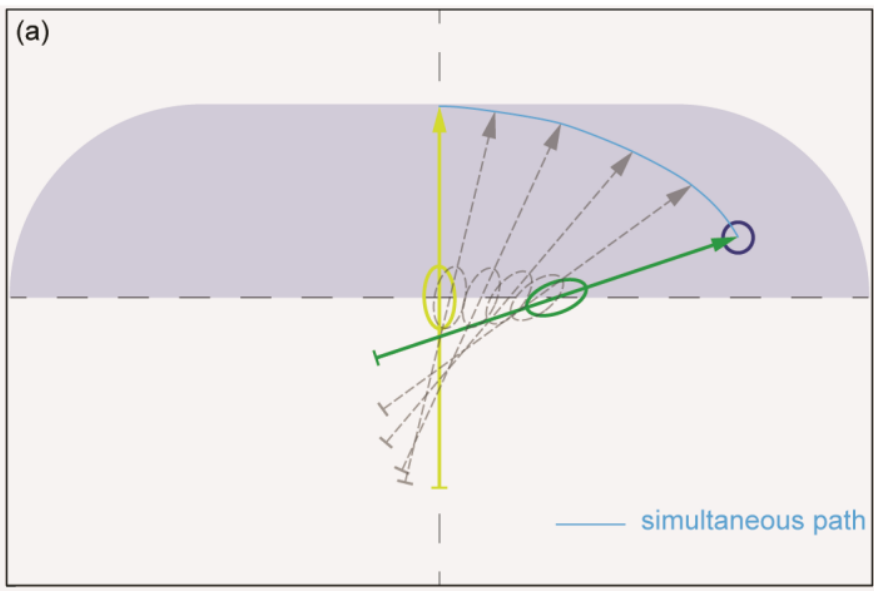

(b)

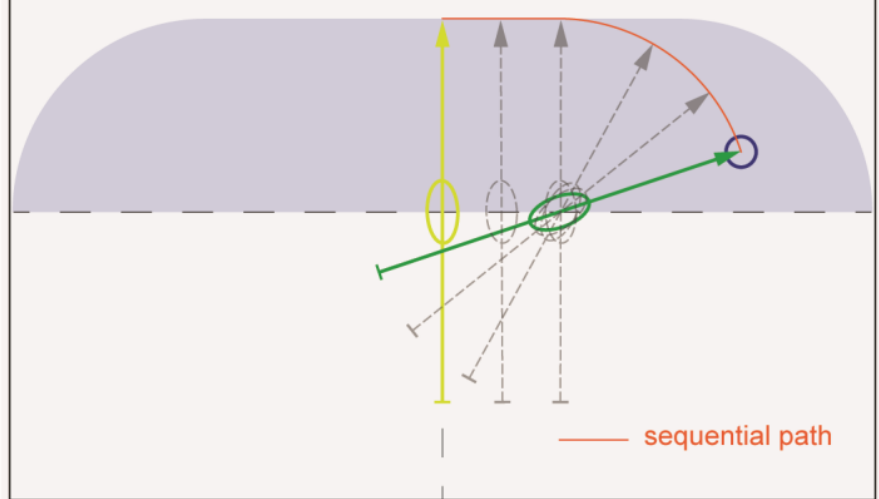

Figure 17: The subject is asked to steer the tip of the arrow into the target circle (blue). The trajectory depends on the strategy chosen. In (a), a simultaneous activation of the two DOF is used, resulting in the shortest path from the starting point to the target. In contrast to this, a sequential path is presented in (b), a valid alternative but not optimal completion of the task. 
However, in the case of target type 3, the subject can use different strategies to complete the task, as these targets require the user to control both DOF provided. The optimal solution and thereby shortest path is to use both DOF simultaneously, as shown in Figure 17a. An alternative approach is to use a sequential activation. An example for this is given in Figure 17b, using first DOF 1 to the horizontal displacement and then DOF 2 for the rotation of the arrow.

Furthermore, a run is defined by a pre-defined set of targets, depending on the complexity of the study. The tasks belonging to a target type are additionally grouped as a series.

An example for a run is provided in Figure 18, with six tasks in the series for target type 1 (targets 1a-1f), six tasks in the series for target type 2 (targets 2a-2f), and twelve tasks for the series of target type 3 (targets $3 a-3 \mid$ ). In contrast to randomly positioned targets, a pre-defined set can be used to compare the performance between subjects or algorithms and also provides a better basis for a statistical analysis of the results. However, the targets should be displayed in random order.

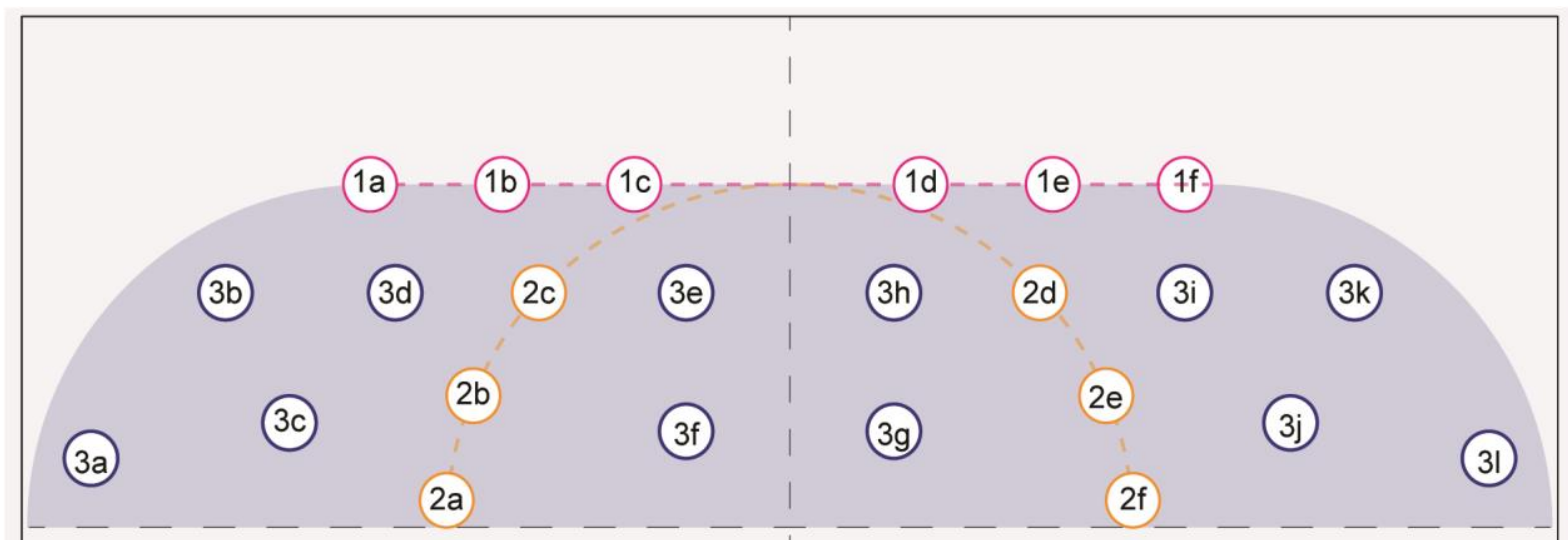

Figure 18: Example for a task set, defining a run. In this run, six tasks are defined for each target type 1 (1a-1f) and target type 2 (2a-2f). For the target type 3 requiring combined activation of the two DOF, a total of 12 tasks is defined (3a-3l). 
Table 1: Basic performance metrics for the Performance Assessment Task

\begin{tabular}{lccl} 
Name & & $\begin{array}{c}\text { Calculated on the } \\
\text { basis of }\end{array}$ & Short Description \\
\hline \hline Task Completion Rate & $\alpha[\%]$ & run or series & Percentage of completed targets \\
\hline Task Completion Time & $t_{c}[s]$ & task & Time to reach and remain in target circle \\
\hline Path Efficiency & $\Gamma[\%]$ & task & $\begin{array}{l}\text { Quotient of optimal path length and } \\
\text { measured path length from start to target } \\
\text { position. }\end{array}$
\end{tabular}

\subsubsection{Performance metrics provided by the performance assessment task}

With the purpose of assessing the control performance, three performance metrics have been defined and are summarized in Table 1.

\subsubsection{Task completion rate}

Considering a full evaluation run, the number of tasks completed in the run is the task completion rate $\alpha_{\text {run }}$. Depending on the study aim and statistical analysis, the completion rate can also be calculated separately for each series in the run $\left(\alpha_{\text {typel }}\right.$, $\left.\alpha_{\text {type2 }}, \alpha_{\text {type3 }}\right)$.

\subsubsection{Task completion time and path efficiency}

Considering each task individually, two additional metrics have been defined. First, the time necessary to complete the task is measured as the completion time $t_{c}$. Second, the trajectory of the arrow's tip $\vec{s}_{\text {trajectory }}(t)=\left[\begin{array}{l}x_{\text {trajectory }}(t) \\ y_{\text {trajectory }}(t)\end{array}\right] \in R^{2}$ from the neutral position to the target circle is recorded. The quotient between the length of the shortest path $\overrightarrow{\mathrm{s}}_{\text {optimal }}(t)=\left[\begin{array}{l}x_{\text {optimal }}(t) \\ y_{\text {optimal }}(t)\end{array}\right] \in R^{2}$ and the length of the measured path $\vec{s}_{\text {trajectory }}(t)$ is defined as the path efficiency: 


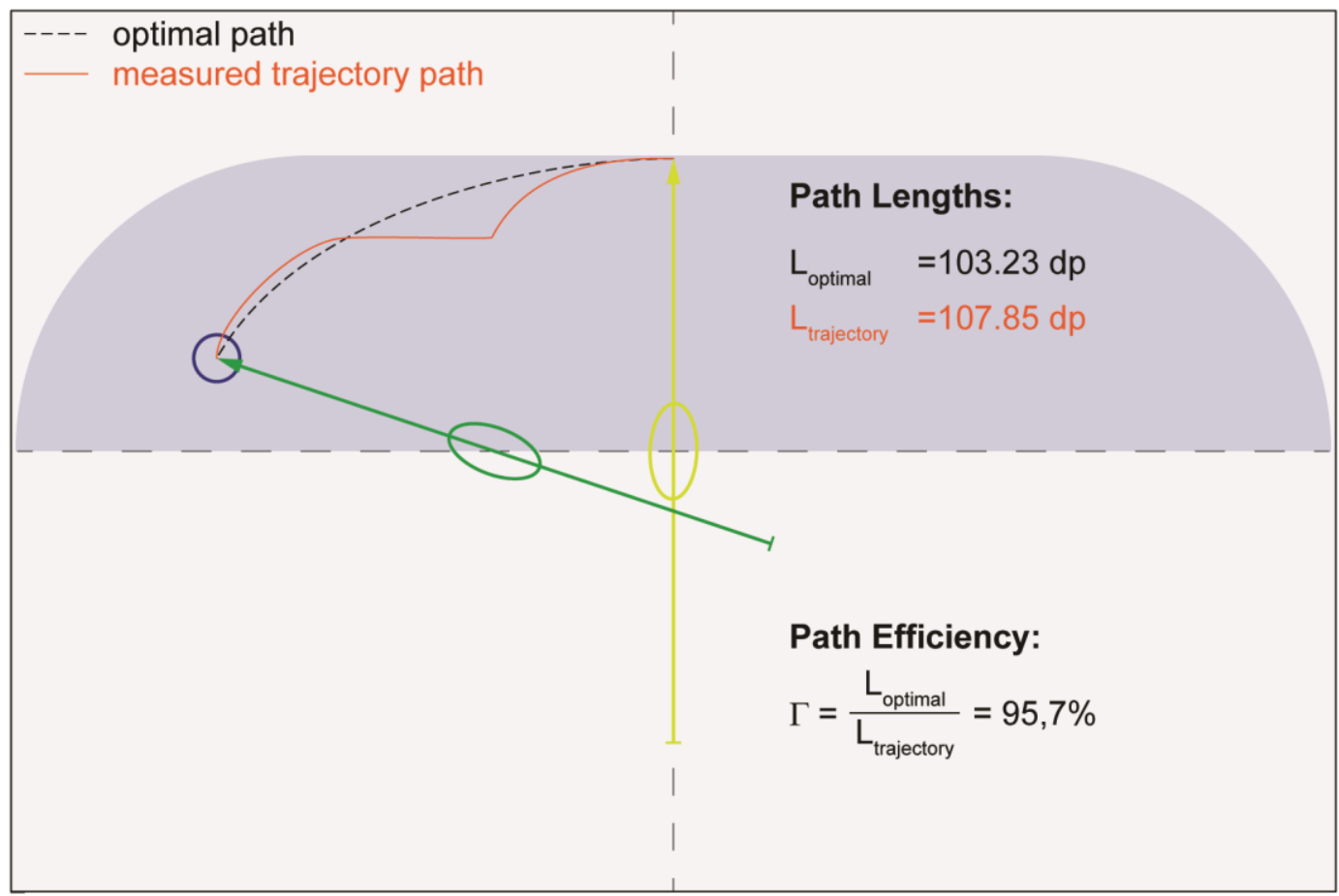

Figure 19: The path efficiency is calculated as a ratio between optimal path length and measured trajectory path length.

$$
\Gamma=\frac{L_{\text {optimal }}}{L_{\text {trajectory }}}=\frac{\int_{0}^{t_{c}} \sqrt{\dot{x}_{\text {optimal }}(t)^{2}+\dot{y}_{\text {optimal }}(t)^{2}} d t}{\int_{0}^{t_{c}} \sqrt{\dot{x}_{\text {trajectory }}(t)^{2}+\dot{y}_{\text {trajectory }}(t)^{2}} d t}
$$

This metric is reported in percentage. An example is presented in Figure 19

These two task-based metrics can then be used to calculate a mean completion time $\bar{t}_{c}$ and mean path efficiency grouped by run $\left(\bar{\Gamma}_{\text {run }}\right)$ or series $\left(\bar{\Gamma}_{\text {type1 }}, \bar{\Gamma}_{\text {type2 }}, \bar{\Gamma}_{\text {type3 }}\right)$, as well as the corresponding standard deviations.

Altogether, the three metrics presented (completion rate, completion time and path efficiency) provide an assessment of both the speed and quality of the control system under evaluation. Furthermore, by calculating path efficiency and completion time within the different series, a comparison of the control performance between 
DOF 1 (target type 1), DOF2 (target type 2) and DOF1+2 (target type 3) can be made.

\subsubsection{Additional metrics}

Besides the above three metrics, other three metrics have been introduced in [83]. All of these are calculated on a task base and are summarized in Table 2.

Due to the given dwelling time, it can happen that the subject reaches the target successfully, but then moves again out of the circle before $T_{d w e l l}$ elapses. The number of these occurrences is measured by the metric Overshoots $k$.

The Throughput $T P$ is used to assess the information the user can deliver through the control system. As introduced in [83], [86], the task difficulty index is defined as

$$
T D I=\log _{2}\left(\frac{A}{d_{\text {circle }}}+1\right) \text { bit }
$$

representing the Shannon extension of Fitts' law [87], [88]. The target amplitude is defined as

$$
A=\left(0.4\left|v_{\text {target }}\right|+0.6\left|\varphi_{\text {target }}\right|\right)^{2} \mathrm{dp},
$$

Table 2: Additional performance metrics for the Performance Assessment Task Name Calculated on

\begin{tabular}{lccl}
\hline Overshoots & $k$ & task & $\begin{array}{l}\text { Number of occurrences that the tip of the arrow hits the } \\
\text { target circle but does not remain for the dwelling time }\end{array}$ \\
\hline Throughput & $T P[\mathrm{bit} / \mathrm{s}]$ & task & $\begin{array}{l}\text { Ratio of targets weighted by difficulty index and } \\
\text { completion time }\end{array}$ \\
\hline Speed & $v[\mathrm{dp} / s]$ & task & $\begin{array}{l}\text { Length of the trajectory from start to target position } \\
\text { divided by completion time }\end{array}$ \\
\hline
\end{tabular}


where $v_{\text {target }}$ and $\varphi_{\text {target }}$ characterize the control angles necessary to reach the presented target. The weights are chosen differently, to account for the more difficult controllability of DOF $2\left(\varphi_{\text {target }}\right)$. Finally, the Throughput is defined as:

$$
T P=\frac{T P I}{t_{c}}
$$

The Speed $v$ is defined as the average speed of the arrows curser. Thus, it is the quotient of measured trajectory path length and completion time:

$$
v=\frac{L_{\text {trajectory }}}{t_{c}} .
$$

\subsubsection{Discussion}

The Virtual Evaluation Paradigm has been presented as a simple and intuitive interface to acquire labeled or unlabeled training data, as well as an online evaluation paradigm for myocontrol algorithms. Specifically, the implemented subject interface is self-explanatory, increasing the acceptance by potential users. This was also confirmed during several studies that used this interface [61], [65], [82]-[85], [89] and that included subjects of different age (14-72 years) as well as subjects with different experience with computer interfaces. None of the subjects had difficulties in understanding the representation or following the prompt. Moreover, the Performance Assessment Task uses the same interface and yielded an overall acceptance by the subjects of the same studies. By separating the task into the three types, also the effect of proportional single DOF activations and simultaneous and proportional activations of both DOF can be investigated. 
Besides the user acceptance of the Performance Assessment Task, the metrics introduced also provide a basis for the analysis and comparison of myocontrol algorithms. While the task completion rate $\alpha$ provides an initial and simple estimate for the general controllability of the myocontrol algorithm, the task completion time $t_{c}$ together with the efficiency coefficient $\Gamma$ provide the basis for a qualitative assessment. Especially the efficiency coefficient accounts for insufficient stability of the control system and the use of simultaneous control of both DOF. Finally, the additional metrics presented in section 2.2.6.3 provide the potential for an in depth analysis for benchmarking different myocontrol systems.

Until now, the VEP and Performance Assessment Task have been used within the PhD project for studies towards myocontrol applications for upper extremities, including two DOF. However, the presented subject interface supports also radial and ulnar deviation of the wrist as a third DOF. Similarly to the flexion and extension being mapped to the horizontal displacement of the arrow, the radial and ulnar deviation can be mapped to a vertical displacement of the arrow. Two joined studies without the Performance Assessment Task, using only flexion/extension and radial/ulnar deviation of the wrist, have already been performed [61], [84]. 


\subsection{Online Regression Control System}

Since a decade, regression techniques have been investigated as an alternative path for more intuitive myocontrol systems for upper extremities. Until now, different methods have been introduced in the academic field, based on Artificial Neural Networks [15], [49], [90], non-negative matrix factorization algorithms [91], and probabilistic methods [86].

However, none of the proposed solutions have been implemented in a real scenario of prosthetic usage, but investigated solely in laboratory conditions. In order to merge the results from academia, the requirements of the industry and the daily needs of the amputees, substantial improvements and extensions are still necessary. In this chapter, promising results towards this final goal are presented.

\subsubsection{Physiological model}

In a prosthetic hand each degree of freedom is controlled by a single actuator, for example one motor for the hand rotation and one motor for hand open and close. In

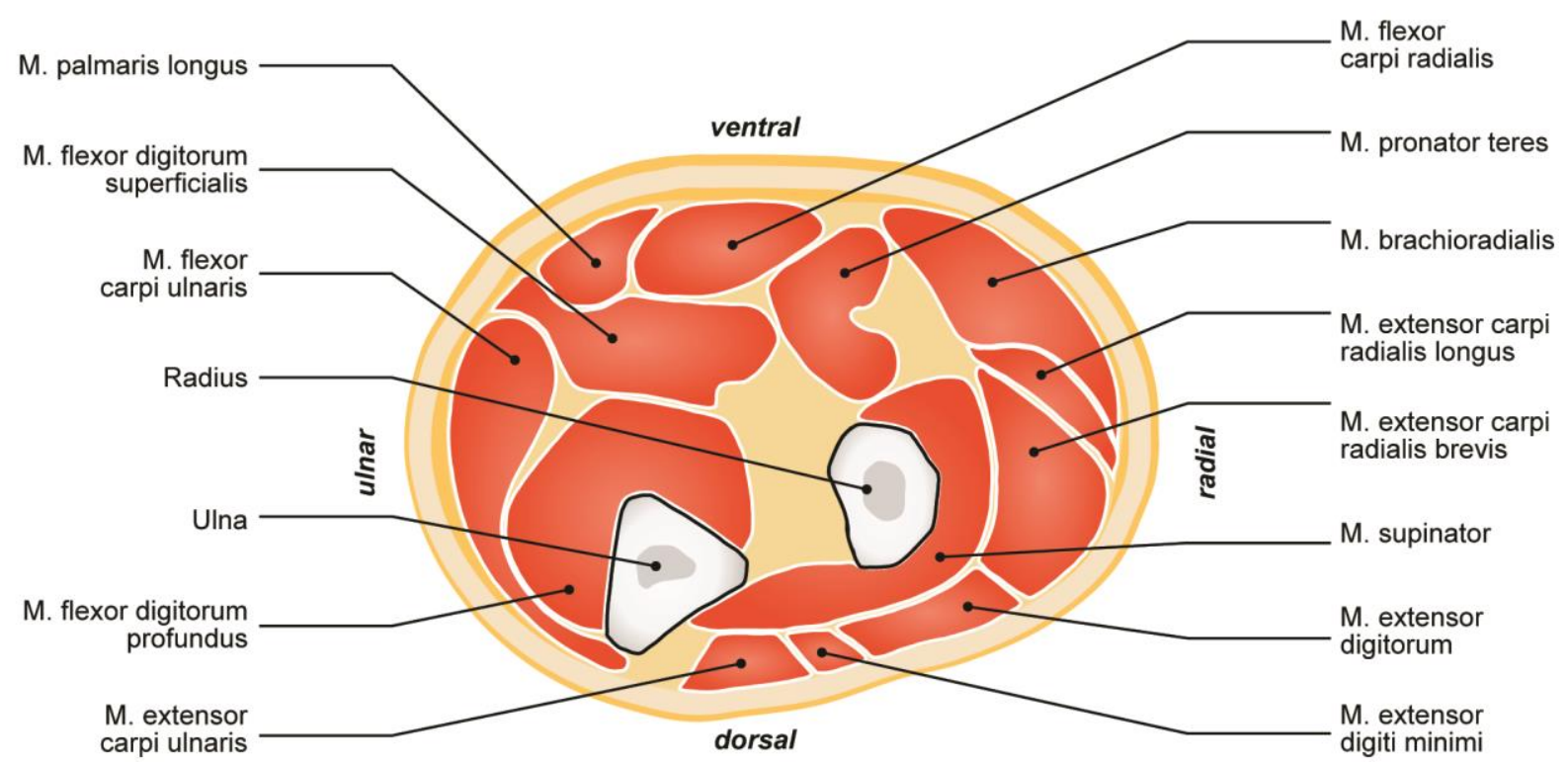

Figure 20: Sagittal cut though the proximal third of the human forearm, showing the presence of 13 muscles in this area. (original work, previously used in [115]) 
contrast, the movements of the human hand are executed by multiple muscles, working together in a synergistic way. The majority of the muscles involved in the movements of the human wrist and hand are located in the distal part of the forearm. Only for some movements of the fingers the muscles are located in the hand itself. In case of the wrist movements considered in this work, it is sufficient to focus on the 13 muscles in the forearm, as shown in Figure 20.

The synergistic activation of a set of muscles involved in a movement is a transformation of a low dimensional and supraspinal signal set from the central nervous system (CNS) into a high dimensional signal set to the muscles. This transformation taking place in the spinal cord has been modeled in [92]-[94], and is described within the so called synergy theory. The synergy concept is composed by two models, the forward and the inverse model. The forward model describes the activation of synergistic muscles from supraspinal motor commands which can be measured on the skin surface by surface EMG signals. The inverse model is the counterpart of the forward model, to obtain the motor commands for prosthesis control from the muscle activations. In the following, the physiological basis of both models is presented in more detail and the online regression control system is derived from both models.

\subsubsection{Forward model}

The application of the synergy model to the problem of controlling the wrist flexion/extension and wrist pronation/supination is presented in Figure 21. According to the model, the intended movement is represented in the motor cortex by control signals for each function (a low dimensional signal set). For each of the four given functions exists an independent control signal $\left(p_{\text {flexion }}(t), \quad p_{\text {extension }}(t), \quad p_{\text {pronation }}(t)\right.$, 


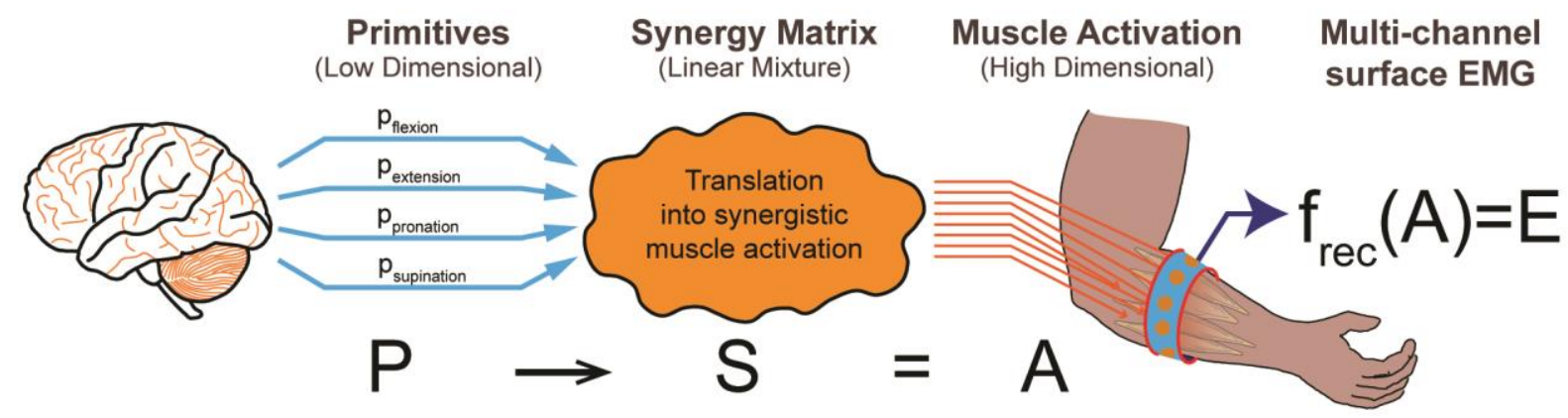

Figure 21: Forward synergy model for the control of wrist flexion/extension and wrist pronation/supination. From the motor cortex the low dimensional motor commands (primitives) are send out to the spinal cord, where they are transformed into the high dimensional muscle activation signals, necessary to execute the intended movement. Finally, the muscle activation is recorded by the multi-channel surface EMG system.

$\left.p_{\text {supination }}(t)\right)$, referred to as primitives. Each primitive indicates the activation of the corresponding function. As physiologically there is no negative activation, the primitives are by definition positive. Finally, the primitives can be combined into a vector representation, with $N$ the number of samples:

$$
P=\left[\begin{array}{c}
p_{\text {flexion }}(t) \\
p_{\text {extension }}(t) \\
p_{\text {pronation }}(t) \\
p_{\text {supination }}(t)
\end{array}\right], P \in R_{0}^{+4 \times N}
$$

The next element of the model is the spinal cord, translating the activations of the primitives into the activations of the synergistic muscles involved in the movement. The muscular activation cannot be negative either (a muscle can only contract actively, but not expand), therefore the image of this mapping function has to be positively defined:

$$
\Omega:{R_{0}^{+4}}^{4} \rightarrow{R_{0}^{+m}}^{m}
$$

with $m$ number of muscles. For the present work, a simplified model was used assuming a linear and time invariant mapping function $\Omega$, equal to the synergy matrix: 


$$
S=\left[\begin{array}{l}
S_{\text {flexion }} \\
S_{\text {extension }} \\
S_{\text {pronation }} \\
S_{\text {supination }}
\end{array}\right], \quad S \in R^{4 \times m} .
$$

From the spinal cord, the muscles are triggered to contract according to the innervation, this is summarized by the muscle activation $A \in R_{0}^{+m \times N}$. Thus, the forward model is summarized by the equation:

$$
S \times P(t)=A(t) .
$$

However, the muscle activation $A(t)$ can only be recorded indirectly by multi-channel surface EMG signals $E(t) \in R^{k \times N}$, with $k$ number of EMG signals recorded. The interrelation between the muscle activation and the EMG recorded on the skin surface is influenced by the characteristics of the tissues between muscles and electrodes [95]-[98]. This transformation function from muscle activation to surface EMG signals can be represented by the function $f_{\text {rec }}$ :

$$
f_{\text {rec }}(A(t))=E(t) .
$$

Taking this into account, the forward synergy model extends to:

$$
f_{\text {rec }}(S \times P(t))=E(t) .
$$

The transformation function $f_{\mathrm{rec}}$ is defined by multiple factors. These include for instance the generative model for the EMG by the motor neurons [95], [98], the filter properties of the tissue between muscles and electrodes [99], or the transfer function 


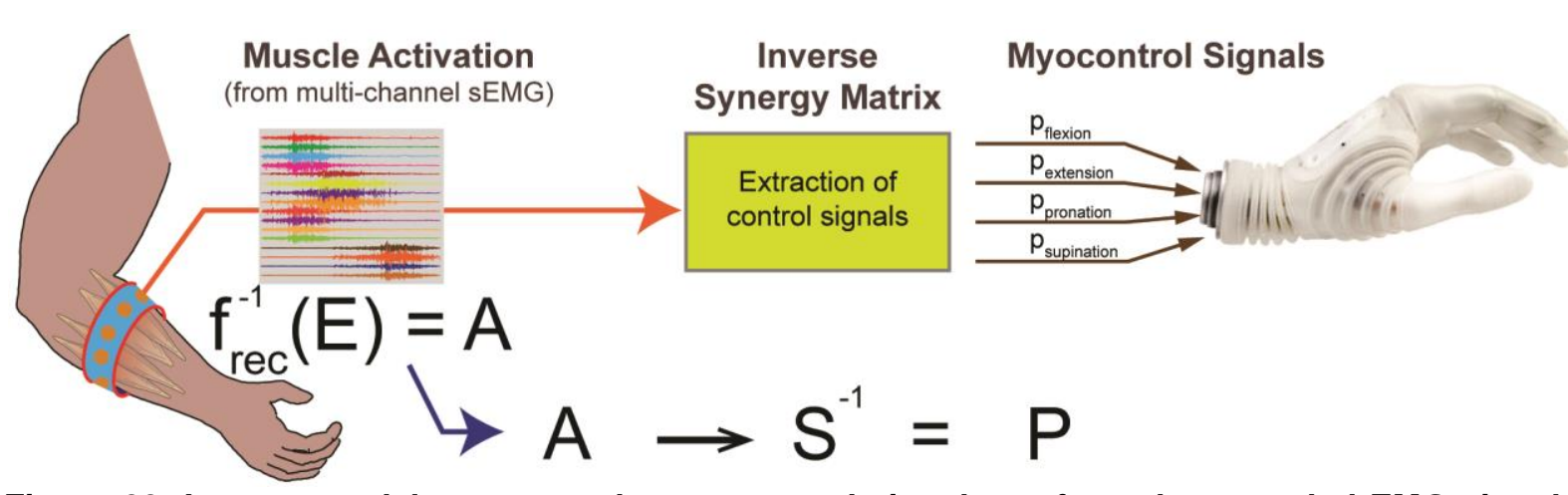

Figure 22: Inverse model to extract the myocontrol signals $C$ from the recorded EMG signals E.

of the electrodes used [95]. However, this generally very complex function $f_{\text {rec }}$ is usually approximated by a linear transformation.

\subsubsection{Inverse model}

The primitives $P$ which are used as motor commands for the prosthesis are extracted from the recorded EMG signals. For this, first the inverse function $f_{\text {rec }}^{-1}$ is obtained in order to estimate the muscle activation $A$ from $E$. Additionally, the inverse synergy matrix $S^{-1}$ has to be found. Finally, primitives $P$ are the product between muscle activation $A$ and the inverse synergy matrix $S^{-1}$. This approach is illustrated in Figure 22.

\subsubsection{Structure of the developed control system}

In order to extract the synergy matrix from recorded EMG data, among other factorization methods, the NMF algorithm [100] has been proposed and used so far just for the offline analysis of recorded EMG data [101], [102]. Specifically, this method has likewise been suggested for the analysis of movements of the upper extremities [14], [90]. Nonetheless, these findings resulted from studies offline and in laboratory conditions [23] including only static muscle activations [14], not accounting for the requirements of prosthesis users in daily life usage. By reconsidering these 


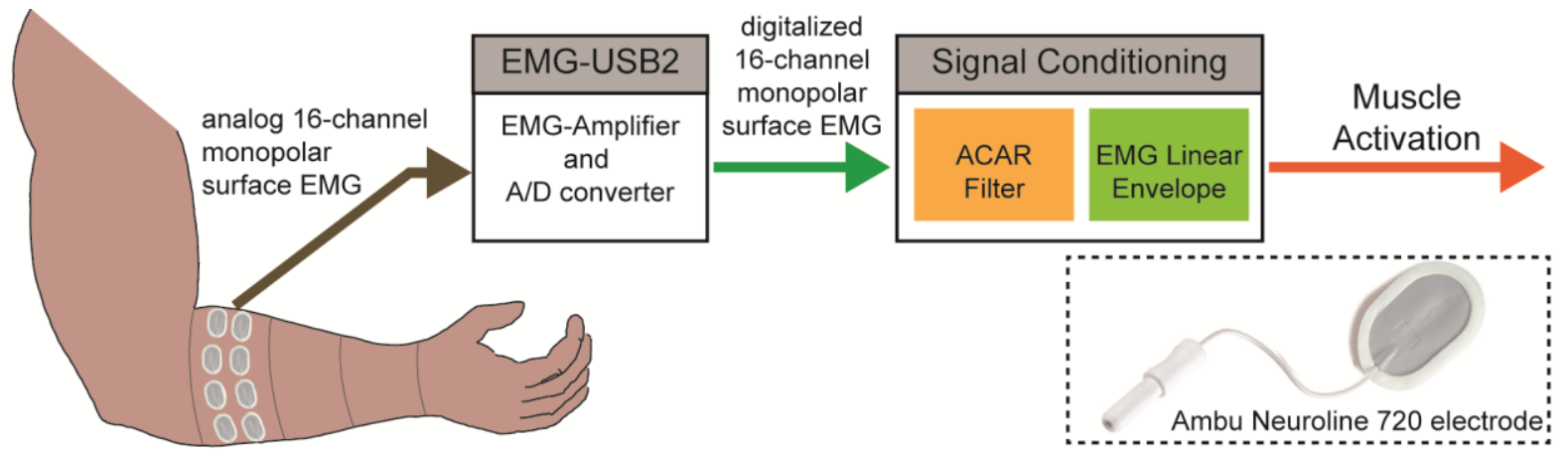

Figure 23: The recording setup used for the EMG signal acquisition. Sixteen electrodes of type Ambu Neuroline 720 (see lower right box) were placed around the forearm in a 2x8 layout. The monopolar signals were acquired and digitalized by a EMG-USB2 amplifier. Finally, the digitalized signal was conditioned to represent the muscle activation in the synergy model (the forward model).

requirements and optimizing each component of the inverse model, the following improvements have been developed and implemented.

\subsubsection{EMG acquisition system}

For the acquisition of the EMG signals, an EMG-USB2 amplifier produced by OT Bioelettronica ${ }^{4}$, Turin, Italy, in combination with 16 Ambu Neuroline $720^{5}$ single use self-adhesive pre-gelled electrodes have been used. The electrodes were placed around the center of the proximal third of the forearm, in two circles each with eight equidistantly placed electrodes, see Figure 23. The EMG was recorded in monopolar mode, filtered with a 3 to $500 \mathrm{~Hz}$ band pass, amplified by a gain of 500 and digitalized at a sampling rate of $2048 \mathrm{~Hz}$ with 16 bit resolution. These digitalized EMG signals $E(t) \in R^{k \times N}, \quad k=16$, is send to the software environment for signal conditioning and extraction of the muscle activation through the forward model.

\footnotetext{
${ }^{4} \mathrm{http}: / /$ www.otbioelettronica.it/

${ }^{5} \mathrm{http} / / /$ www.ambu.com/corp/products/patient_monitoring_and_diagnostics/product/neuroline_720prod14061.aspx
} 


\subsubsection{Estimation of the muscle activation from the surface EMG}

As introduced in section 2.3.1.1, the muscles are represented in the model by their muscle activation $A \in R_{0}^{+k \times N}$. Hence, this activation signal has to be estimated from the surface EMG $E(t) \in R^{k \times N}$, denoted in the inverse model by the function $f_{\text {rec }}^{-1}$. In the proposed control system, $f_{\text {rec }}^{-1}$ is characterized by two components: filtering the raw EMG using the ACAR filter and calculating the EMG linear envelope. This is summarized as the signal conditioning in Figure 23 and described in detail in the following two subsections.

\subsection{Signal pre-processing for improving selectivity}

The first component of the signal conditioning is the signal pre-processing. Ideally, the activation of a target muscle can be recorded more selectively using invasive intra-muscular needle EMG electrodes [95]. However, this technique is invasive and the issues related to the bio-compatibility of the implants with the tissue have not been solved yet for clinical applications. Therefore, it is currently more viable to record the muscle activation using surface EMG electrodes. However, recording muscle signals from the skin has drawbacks for the synergy model. First, surface EMG signals are more susceptible to noise. Second, surface EMG electrodes pick signals from neighboring muscles. This phenomenon is known as crosstalk and poor selectivity.

To reduce the effects of noise and especially to record more selectively, the Adaptive Common Average Reference Filtering (ACAR) has been developed, as described in chapter 2.1. This filter is applied to the recorded EMG signal $E(t) \in R^{k \times N}$, providing the optimized EMG signal $E_{\mathrm{ACAR}}(t) \in R^{k \times N}$ used for the estimation of the muscle activation in the next step. 


\subsection{Estimation of muscle activation using EMG linear envelope}

One criterion of the synergy model is that the muscle activation $A(t)$ by definition has a positive value range, because muscles can only contract actively. The extension of a muscle is always done passively by an antagonistic muscle.

Yet, the raw EMG signal is a stochastic signal with zero mean [95], therefore it does not satisfy this requirement. The same applies for the ACAR filtered EMG signal $E_{\mathrm{ACAR}}(t)$. In order to have an estimate for the muscle activation as described in the model, an additional feature extraction stage is necessary to extract a valid estimate for the muscle activation $A \in R_{0}^{+^{k \times N}}$ from the ACAR filtered $E_{\mathrm{ACAR}}(t)$, with a positive range of values.

The feature used was the EMG linear envelope. This feature is often used in measurements during locomotion to represent the activation of muscles involved during gait [103]-[105]. The EMG linear envelope was calculated on a channel basis, by applying a full-wave rectifier to $E_{\mathrm{ACAR}}(t)$, followed by a 3-rd order low-pass Butterworth filter with a $3 \mathrm{~dB}$-cut-off frequency of $5 \mathrm{~Hz}$. It was observed that the low-

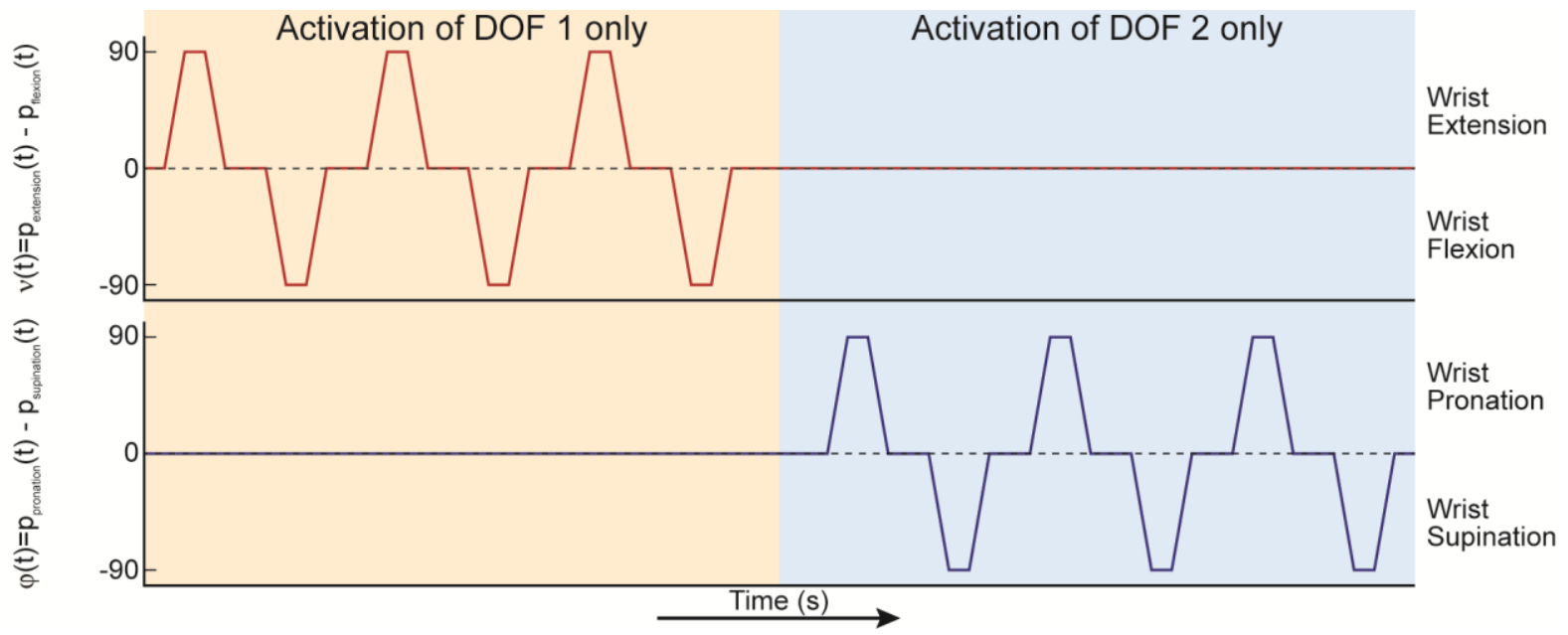

Figure 24: An example for a prompt used in the calibration phase for the recording of sample EMG data. 
pass filtering stage could introduce negative values due to overshoots. These remaining negative values were removed using a final half-wave rectification stage, to comply with the non-negative constraint of the model. Finally, this signal $A(t) \in R_{0}^{+}$ representing the muscle activation was used as the input signal for the synergy model. However, as the muscle activation is calculated from all EMG channels available, the number of muscle activation was equal to the number of EMG channels, thus $m=k$.

\subsubsection{Calibration data}

A calibration of the system has to be performed in order to extract the synergy matrix and obtain the primitives from the inverse model. For this, sample calibration data has to be recorded using the provided recording system. The Virtual Evaluation Paradigm in the Guided calibration mode is used, prompting the subject to perform a series of pre-defined movements. The provided prompt includes single activations of the four functions, grouped by the corresponding DOF. An example for this prompt is visualized in Figure 24. In the first part, an alternating series of wrist flexion and extension (DOF 1) is performed and repeated three times. In the second part, only the wrist rotation (DOF 2 ) is activated in the same alternating manner. Although the EMG signals and the presented prompts are saved together, the prompts cannot be considered as labels. The prompts are only recommendations, as the actual muscle activations performed by the subjects can differ. The only importance is that only a single DOF is activated. This data is called calibration data.

The recorded calibration data is in contrast to pattern recognition methods, where the EMG signals have to be recorded with exactly matching labels [20], [23], [106], 
resulting in training data. This would only be the case for the Free calibration mode, where the actual kinematics are recorded as labels.

\subsubsection{Extracting the synergy matrix using Non-negative Matrix Factorization}

In the Forward model, the relation between the primitives and the muscle activation is encoded by the mixing synergy matrix.

Hence, the correct estimation of this matrix $S$ provides the key to solve and apply the inverse model (see Figure 25). For the estimation of the synergy matrix the Nonnegative Matrix Factorization (NMF) algorithm has been shown to be an efficient and promising method [14]. The input for this algorithm is a sample data set of muscle activations $A \in R_{0}^{+^{m \times N}}$, extracted from the surface EMG signals of the calibration data. Using the NMF algorithm, this matrix can be factorized into the two matrices $A^{m \times N}=S^{m \times 4} \times P^{4 \times N}$, or more precisely:

$$
\left[\begin{array}{c}
a^{1} \\
\vdots \\
a^{m}
\end{array}\right]^{m \times N}=\left[\begin{array}{llll}
s_{\text {flexion }} & s_{\text {extension }} & s_{\text {pronation }} & s_{\text {supination }}
\end{array}\right]^{m \times 4} \times\left[\begin{array}{c}
p_{\text {flexion }} \\
p_{\text {extension }} \\
p_{\text {pronation }} \\
p_{\text {supination }}
\end{array}\right]^{4 \times N}
$$

While the factorization is primarily targeted to estimate the mixing matrix $S^{m \times 4}$, the

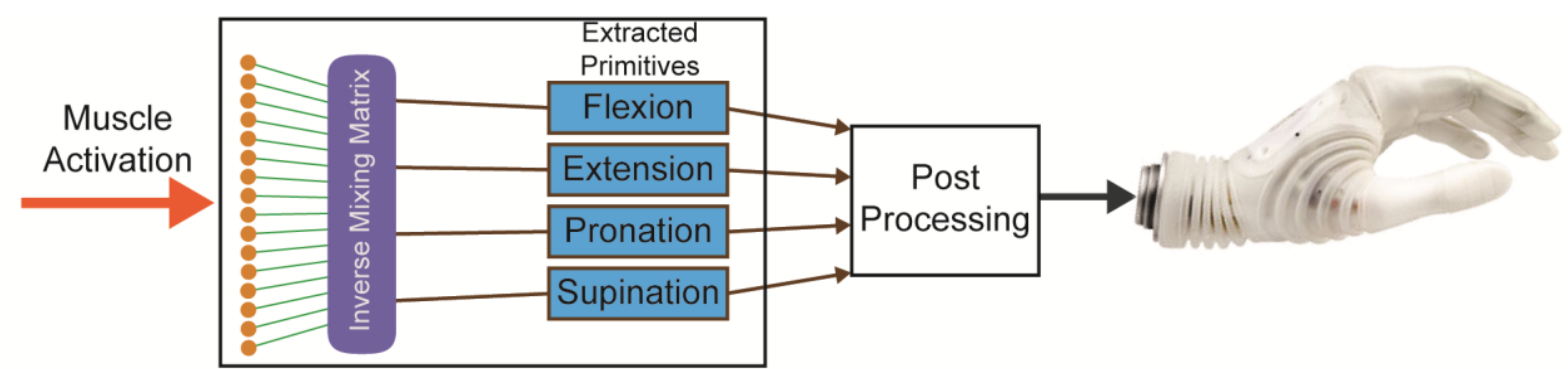

Figure 25: The input of the regression system is the muscle activation estimation, provided by the signal conditioning unit. The core of the regression system is the mixing matrix, calculated from the calibration data. Using this matrix, the primitives were extracted from the muscle activation. 
NMF algorithm also returns the primitives $P^{4 \times N}$ corresponding to the calibration data.

The NMF algorithm is indeterminate in regard to the order of the components. This indeterminacy entails that the factorization algorithm return $P^{4 \times N}$ and $S^{m \times 4}$ in a randomly permutated order $\left[\begin{array}{llll}A & B & C & D\end{array}\right]$ :

$$
\left[\begin{array}{c}
a^{1} \\
\vdots \\
a^{m}
\end{array}\right]^{m \times N}=\left[\begin{array}{llll}
s_{\mathrm{A}} & s_{\mathrm{B}} & s_{\mathrm{C}} & s_{\mathrm{D}}
\end{array}\right]^{m \times 4} \times\left[\begin{array}{c}
p_{\mathrm{A}} \\
p_{\mathrm{B}} \\
p_{\mathrm{C}} \\
p_{\mathrm{D}}
\end{array}\right]^{4 \times N} .
$$

However, instead of using the factorization approach on the global scale, a DOFwise processing of the calibration data is preferred.

\subsection{The DOF-wise factorization approach}

In order to optimize the algorithm towards a meaningful factorization and simplify the identification of the factorized components, a "divide and conquer" approach [91] is used. This approach divides the factorization along the two DOF, rather than solving the problem for all DOF simultaneously. This dividing step is also the reason for the pre-defined set of calibration EMG data recorded previously (see section 2.3.2.3). Firstly, in the dividing step the recorded muscle activation $A^{m \times N}$ is separated into two data sets, each containing the activation of a single DOF.

$$
A^{m \times N}=\left[A_{\mathrm{DOF} F}^{m \times L}, A_{\mathrm{DOF}}^{m \times M}\right] \text {, with } L, M>0 \text { and } L+M=N .
$$

and likewise the factorization is separated into two factorizations 


$$
\begin{gathered}
{\left[\begin{array}{ll}
s_{\mathrm{DOF} 1, \mathrm{~A}} & s_{\mathrm{DOF} 1, \mathrm{~B}}
\end{array}\right]^{m \times 2} \times\left[\begin{array}{c}
p_{\mathrm{DOF} 1, \mathrm{~A}} \\
p_{\mathrm{DOF} 1, \mathrm{~B}}
\end{array}\right]^{2 \times L}=\left[\begin{array}{c}
a_{\mathrm{DOF} 1}^{1} \\
\vdots \\
a_{\mathrm{DOF} 1}^{m}
\end{array}\right]^{m \times L} \text { and }} \\
{\left[\begin{array}{ll}
s_{\mathrm{DOF} 2, \mathrm{~A}} & s_{\mathrm{DOF} 2, \mathrm{~B}}
\end{array}\right]^{m \times 2} \times\left[\begin{array}{c}
p_{\mathrm{DOF} 2, \mathrm{~A}} \\
p_{\mathrm{DOF} 2, \mathrm{~B}}
\end{array}\right]^{2 \times M}=\left[\begin{array}{c}
a_{\mathrm{DOF} 2}^{1} \\
\vdots \\
a_{\mathrm{DOF} 2}^{m}
\end{array}\right]^{m \times M} .}
\end{gathered}
$$

By applying the NMF algorithm to both DOF separately, the indeterminacy is reduced to the level of each DOF; the two data sets are "conquered" separately. Nonetheless, each factorization provides a component for the complete mixing matrix $S^{m \times 4}$. What is left to build this complete mixing matrix is to identify the permutation matrices $\Pi_{\mathrm{DOF} 1}$ and $\Pi_{\mathrm{DOF} 2}$, resolving the order of the factorization components:

$$
\begin{aligned}
& S=\left[\begin{array}{llll}
s_{\text {flexion }} & S_{\text {extension }} & S_{\text {pronation }} & S_{\text {supination }}
\end{array}\right] \\
& S=\left[\left[\begin{array}{ll}
s_{\mathrm{DOF} 1, \mathrm{~A}} & s_{\mathrm{DOF} 1, \mathrm{~B}}
\end{array}\right] \times\left[\Pi_{\mathrm{DOF} 1}\right]^{2 \times 2}, \quad\left[\begin{array}{ll}
s_{\mathrm{DOF} 2, \mathrm{~A}} & s_{\mathrm{DOF} 2, \mathrm{~B}}
\end{array}\right] \times\left[\Pi_{\mathrm{DOF} 2}\right]^{2 \times 2}\right]
\end{aligned}
$$

\subsection{Resolving the indeterminacy}

Taking into account the prior knowledge about the labels of the performed calibration movements, this indeterminacy can be resolved in a second step. More precisely, it is possible to match the columns of the matrix $\left[\begin{array}{l}p_{\mathrm{DOF} 1, \mathrm{~A}} \\ p_{\mathrm{DOF} 1, \mathrm{~B}}\end{array}\right]$ with $v(t)$ - the labels of DOF 1 - and the columns of $\left[\begin{array}{c}p_{\mathrm{DOF} 2, \mathrm{~A}} \\ p_{\mathrm{DOF} 2, \mathrm{~B}}\end{array}\right]$ with $\varphi(t)$ - the labels of DOF 2.

For this matching process, the label for DOF $1, v(t)$, is separated into two sublabels, each with positive value ranges: 


$$
\begin{aligned}
& v^{+}(t)=\left\{\begin{array}{ll}
v(t) & , \text { when } v(t) \geq 0 \\
0 & , \text { when } v(t)<0
\end{array},\right. \\
& v^{-}(t)= \begin{cases}0 & , \text { when } v(t) \geq 0 \\
-v(t) & , \text { when } v(t)<0\end{cases}
\end{aligned}
$$

For a right handed subject, $v^{+}(t)$ is $>0$ during wrist flexion and $v^{-}(t)$ is $>0$ during wrist extension (see Figure 26). Next, the pair-wise cross correlation $\operatorname{cross}(A, B)$ between the labels and the identified primitives is calculated

$$
\begin{aligned}
& \chi_{\mathrm{A}, \mathrm{v}+}=\operatorname{cross}\left(p_{\mathrm{DOF} 1, \mathrm{~A}}, v^{+}(t)\right) \\
& \chi_{\mathrm{B}, \mathrm{v}+}=\operatorname{cross}\left(p_{\mathrm{DOF} 1, \mathrm{~B}}, v^{+}(t)\right) \\
& \chi_{\mathrm{A},-}=\operatorname{cross}\left(p_{\mathrm{DOF} 1, \mathrm{~A}}, v^{-}(t)\right) \\
& \chi_{\mathrm{B}, \mathrm{v}-}=\operatorname{cross}\left(p_{\mathrm{DOF} 1, \mathrm{~B}}, v^{-}(t)\right)
\end{aligned}
$$

and used to select the corresponding permutation matrix $\Pi_{\mathrm{DOF} 1}$ :

$$
\Pi_{\mathrm{DOF} 1}=\left\{\begin{array}{ll}
{\left[\begin{array}{ll}
1 & 0 \\
0 & 1
\end{array}\right]} & \text {, when } \chi_{\mathrm{A}, \mathrm{v}+}>\chi_{\mathrm{A}, \mathrm{v}-} \wedge \chi_{\mathrm{B}, \mathrm{v}-}>\chi_{\mathrm{B}, \mathrm{v}+} \\
{\left[\begin{array}{ll}
0 & 1 \\
1 & 0
\end{array}\right]} & \text {, when } \chi_{\mathrm{A}, \mathrm{v}+}<\chi_{\mathrm{A}, \mathrm{v}-} \wedge \chi_{\mathrm{B}, \mathrm{v}-}<\chi_{\mathrm{B}, \mathrm{v}+}
\end{array} .\right.
$$

In this manner, the extracted primitives and the labels are matched. An example for this matching process is given in Figure 26.

However, the primitives identified by the factorization process are of arbitrary units and value range. By applying a scaling factor $\left(\alpha_{\mathrm{DOF} 1}^{+}, \alpha_{\mathrm{DOF} 1}^{-}\right)$to each of the extracted primitives, they can be scaled to the value range of the wrist angles, as used in the label $v(t)$. 


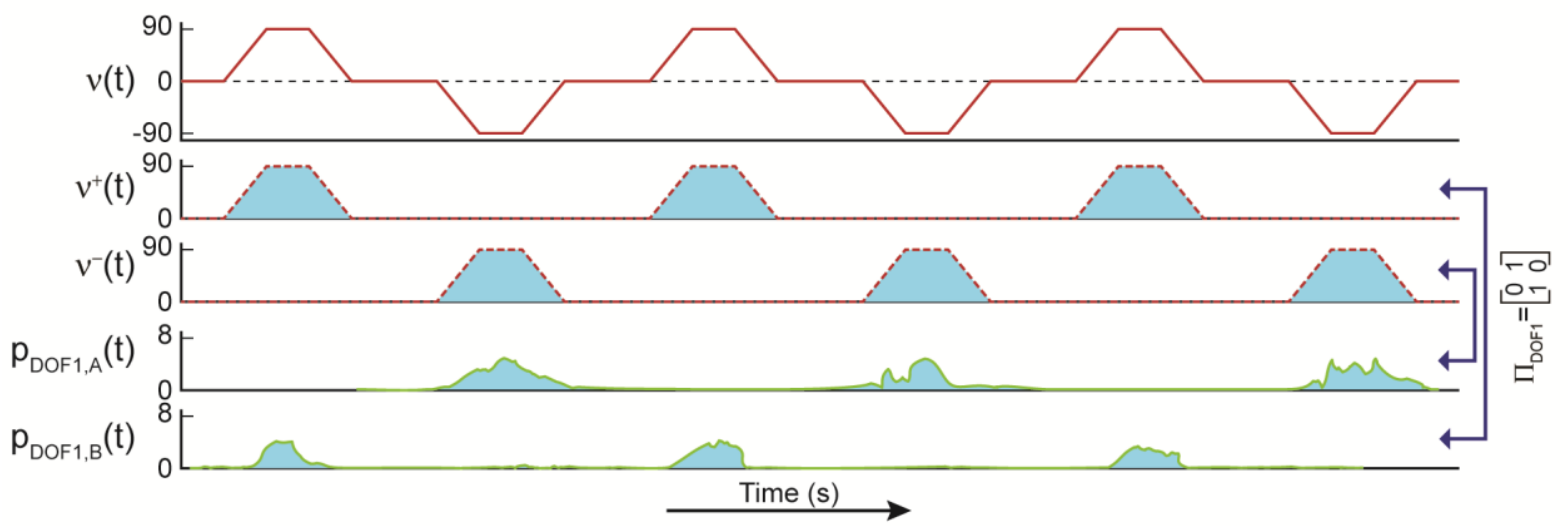

Figure 26: Resolving the indeterminacy for DOF 1: The label $v(t)$ for the recorded data is separated into the two function components $v^{+}(t)$ and $v^{-}(t)$. Using the cross correlation between these components and the extracted primitives the correct permutation matrix is identified.

To automatically calculate these scaling factors, the area under the curve of the primitives extracted in the calibration is used. Thus, the scaling factor is defined as the ratio of the label's area under the curve and the extracted primitive's area under the curve, for the intervals of a movement being performed. In Figure 26 the area under the curve is colored in light blue. For the given example the factors are calculated as:

$$
\alpha_{\mathrm{DOF} 1}^{+}=\frac{\int_{v^{+}(t)>0} v^{+}(t) d t}{\int_{v^{+}(t)>0} p_{\mathrm{DOF} 1, \mathrm{~B}}(t) d t} \quad \alpha_{\mathrm{DOF} 1}^{-}=\frac{\int_{v^{-}(t)>0} v^{-}(t) d t}{\int_{v^{-}(t)>0} p_{\mathrm{DOF} 1, \mathrm{~A}}(t) d t}
$$

In this manner, the permutation matrix $\Pi_{\mathrm{DOF} 1}$ and the scaling factors $\alpha_{\mathrm{DOF} 1}^{+}$and $\alpha_{\mathrm{DOF} 1}^{-}$ for DOF 1 are identified and the indeterminacy for the flexion and extension angles is resolved. Identically, the permutation matrix $\Pi_{\mathrm{DOF} 2}$ and the scaling factors $\alpha_{\mathrm{DOF} 2}^{+}$and $\alpha_{\mathrm{DOF} 2}^{-}$for DOF 2 can be identified from $\left[\begin{array}{c}p_{\mathrm{DOF} 2, \mathrm{~A}} \\ p_{\mathrm{DOF} 2, \mathrm{~B}}\end{array}\right]$ and $\varphi(t)$ in order to resolve the indeterminacy for the pronation and supination angles. 


\subsubsection{Estimation of control signals using the synergy matrix}

In the previous step, the synergy matrix $S=\left[\begin{array}{lllll}s_{\text {flexion }} & S_{\text {extension }} & S_{\text {pronation }} & s_{\text {supination }}\end{array}\right]$ has been identified from recorded calibration data, using the NMF algorithm. In addition, the scaling factors $\left[\begin{array}{llll}\alpha_{\mathrm{DOF} 1}^{+} & \alpha_{\mathrm{DOF} 1}^{-} & \alpha_{\mathrm{DOF} 2}^{+} & \alpha_{\mathrm{DOF} 2}^{-}\end{array}\right]$were determined. These parameters are now applied to the inverse model to calculate the primitives from new and unseen EMG signals online.

\subsection{Calculation of the inverse synergy matrix}

The inverse synergy model to extract control signals $C(t)=\left[\begin{array}{llll}c_{\text {flexion }} & c_{\text {extension }} & c_{\text {pronation }} & c_{\text {supination }}\end{array}\right]^{T}$ from the muscle activation $A(t)$ is given by the equation

$$
C(t)=S^{-1} \times A(t)
$$

The previous step only returned the forward synergy matrix $S$, which is a nonquadratic matrix with all entries $\in R_{0}^{+}$. A non-quadratic matrix does not have an inverse, but instead the pseudo inverse $S^{+}$can be calculated, using the MoorePenrose algorithm [107], [108]. Similarly to the synergy matrix which has been calculated DOF-wise, the matrix $S^{\text {inv }}$ for the inverse model is calculated separately for each DOF, too. This can be written as

$$
S^{\text {inv }}=\left[\begin{array}{l}
s_{\text {flexion }}^{\text {inv }} \\
S_{\text {exv }}^{\text {invion }} \\
S_{\text {pronation }}^{\text {inv }} \\
S_{\text {supination }}^{\text {inv }}
\end{array}\right]=\left[\left[\begin{array}{l}
s_{\text {flexion }} \\
S_{\text {extension }}
\end{array}\right]^{+}\left[\begin{array}{l}
s_{\text {pronation }} \\
s_{\text {supination }}
\end{array}\right]^{+}\right] \in R^{m \times 4} .
$$




\subsection{Application to newly acquired EMG signals}

In an online application, the control signal is extracted from new EMG data samples $X_{\text {EMG }}(t) \in R^{k \times U}$, acquired constantly in blocks of $U$ samples using the provided hardware.

The steps necessary to estimate the control signal $C(t)$ and finally the wrist angle estimates $v(t)$ and $\varphi(t)$ are illustrated in Figure 27.

In a first step, the EMG muscle activation is estimated by applying the ACAR filter and calculating the EMG linear envelope as described in section 2.3.2.2, resulting in $X_{\text {Activation }}(t) \in R^{m \times U}$. Next, the inverse synergy matrix $S^{\text {inv }}$ is applied to the muscle activation. As a result, the primitives for the four functions are returned:

$$
P(t)=\left[\begin{array}{c}
p_{\text {flexion }} \\
p_{\text {extension }} \\
p_{\text {pronation }} \\
p_{\text {supination }}
\end{array}\right]=S^{\text {inv }} \times X_{\text {Activation }}(t)
$$

The primitives are of arbitrary unit and in order to use the primitives as a control signal $C(t)$, the scaling factors $\left[\begin{array}{llll}\alpha_{\mathrm{DOF} 1}^{+} & \alpha_{\mathrm{DOF} 1}^{-} & \alpha_{\mathrm{DOF} 2}^{+} & \alpha_{\mathrm{DOF} 2}^{-}\end{array}\right]$have to be applied:

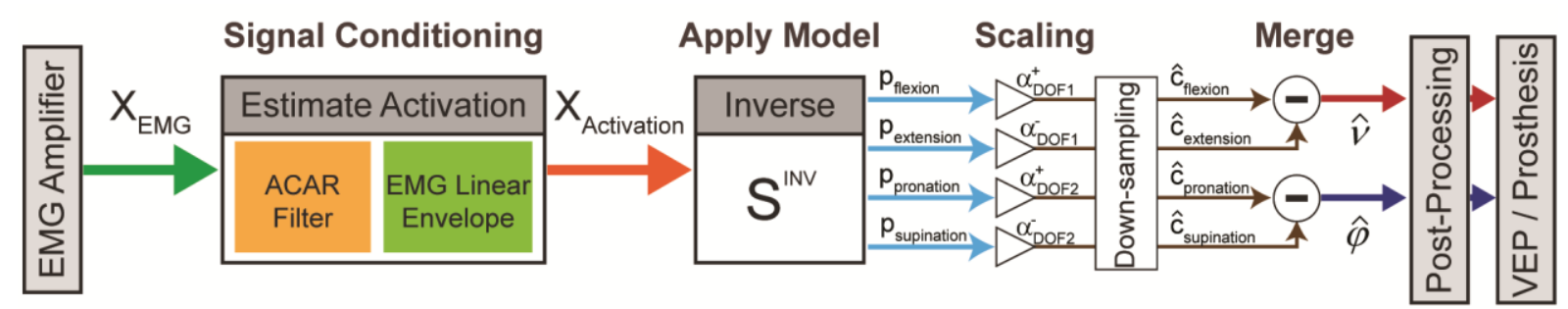

Figure 27: Online processing chain to estimate the control signals. New EMG data is acquired by the provided hardware and the muscle activation is estimated using the ACAR filter and the EMG linear envelope. The resulting muscle activation is multiplied with the inverse synergy matrix and the resulting primitives are scaled using the previously identified scaling factors. Finally, the control signals are down sampled and the difference is calculated. 


$$
C(t)=\left[\begin{array}{cccc}
\alpha_{\mathrm{DOF} 1}^{+} & \cdots & 0 \\
\vdots & \alpha_{\mathrm{DOF} 1}^{-} & & \vdots \\
& \multicolumn{2}{c}{\alpha_{\mathrm{DOF} 2}^{+}} & \alpha_{\mathrm{DOF} 2}^{-}
\end{array}\right] \times P(t)
$$

In the processing chain until now, the high sampling rate of $2048 \mathrm{~Hz}$ originating from the original EMG signal $X_{\mathrm{EMG}}(t)$ is kept. This oversampling is now used to remove outliers in the estimate $C(t)$, by down-sampling the signal. The down-sampling is performed by short time windowing (window length 82 sample $\approx 40 \mathrm{~ms}$ ) and averaging. The resulting sampling rate of the down-sampled estimate $\hat{C}(t)$ is about $25 \mathrm{~Hz}$.

Both the Virtual Evaluation Paradigm and the prosthetic hand require the two angles of the DOF as input. These angles can be calculated by using the DOF-wise difference of the control signal. This can also be written as:

$$
\left[\begin{array}{l}
\hat{v}(t) \\
\hat{\varphi}(t)
\end{array}\right]=\left[\begin{array}{cccc}
1 & -1 & 0 & 0 \\
0 & 0 & 1 & -1
\end{array}\right] \times\left[\begin{array}{l}
\hat{c}_{\text {flexion }}(t) \\
\hat{c}_{\text {extension }}(t) \\
\hat{c}_{\text {pronation }}(t) \\
\hat{c}_{\text {supination }}(t)
\end{array}\right]
$$

\subsection{Post-processing}

For an improved controllability in online experiments, a post-processing of the calculated estimation has been added. This additional step is added to ensure a more stable and reliable estimate, as well as to implement both position control and velocity control, based on the output of the regression system. 
As indicated in Figure 28, the post-processing is separated into two components, plus an optional third integration component. The first component is a validity check for the estimated angles, represented by a non-linear transformation function. This is implemented using thresholds $\left(\hat{v}_{\text {min }}^{+}, \hat{v}_{\text {min }}^{-}, \hat{\varphi}_{\min }^{+}, \hat{\varphi}_{\text {min }}^{-}\right)$and clipping values $\left(\hat{v}_{\text {clip }}^{+}, \hat{v}_{\text {clip }}^{-}, \hat{\varphi}_{\text {clip }}^{+}\right.$, $\left.\hat{\varphi}_{\text {clip }}^{-}\right)$and can be written as:

$$
\hat{v}=\left\{\begin{array}{ll}
0 & , \text { when } \hat{v}_{\text {min }}^{-}<\hat{v}<\hat{v}_{\text {min }}^{+} \\
\hat{v}_{\text {clip }}^{-} & , \text {when } \hat{v}<\hat{v}_{\text {clip }}^{-} \\
\hat{v}_{\text {clip }}^{+} & , \text {when } \hat{v}>\hat{v}_{\text {clip }}^{+} \\
\hat{v} \quad, \text { otherwise }
\end{array} \quad \text { and } \quad \hat{\varphi}=\left\{\begin{array}{ll}
0 \quad, \text { when } \hat{\varphi}_{\text {min }}^{-}<\hat{\varphi}<\hat{\varphi}_{\text {min }}^{+} \\
\hat{\varphi}_{\text {clip }}^{-}, \text {when } \hat{\varphi}<\hat{\varphi}_{\text {clip }}^{-} \\
\hat{\varphi}_{\text {clip }}^{+}, \text {, when } \hat{\varphi}>\hat{\varphi}_{\text {clip }}^{+} \\
\hat{\varphi} \quad \text {, otherwise }
\end{array}\right. \text {. }\right.
$$

The threshold is used to reduce jittering in case of noise or weak muscle activations in the range $\hat{v}_{\text {min }}^{-}<0<\hat{v}_{\text {min }}^{+}$and $\hat{\varphi}_{\text {min }}^{-}<0<\hat{\varphi}_{\text {min }}^{+}$while the clipping avoids estimates exceeding the value range (Figure 28, Validity Check).

The second component is an FIR filter with linear properties to smoothen the output signal. The filter length $N$ is selected based on the subject's skill and for the filter coefficients applies the restriction $\sum_{i^{\prime}=0}^{N} b_{i}=1$. The filters for the two DOF use the same settings. To not reduce the fast response behavior of the system, it is suggested to

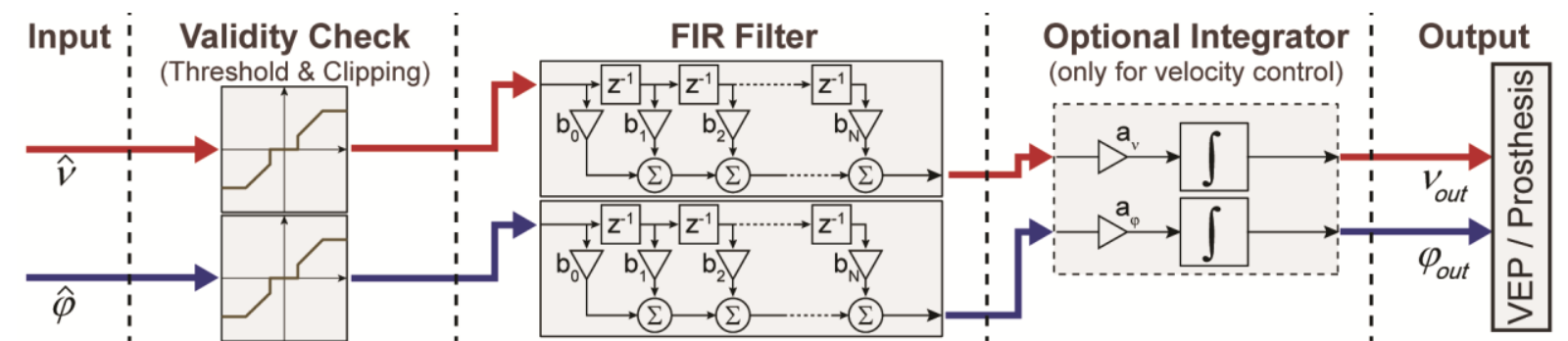

Figure 28: Structure of the non-linear post-processing. In the first component, the estimate is sanitized using a threshold and clipping stage. Weak activations close to zero are suppressed and overshoots are clipped. Only if the estimates are within the borders, they pass proportionally. The second component is an FIR filter to stabilize the output. Finally, the optional integrator as third component can be used to switch from position control mode to velocity control mode. 
select the coefficients according to the rule $b_{0}>b_{1}>\ldots>b_{N}$. Especially for unexperienced subjects, this step can improve the initial control experience, while with increasing subject skill the filter length can then be reduced.

The output of the FIR stage is the control signal used in position mode. In this context, position control refers to the direct mapping of the user's movement intention to the prosthetic hand or VEP. A prosthetic hand for instance mirrors the movements of the user, and in the VEP the arrow position represents the estimated wrist angles. This is equal to the control provided by a computer mouse, where the relative position of the mouse is related to the relative position of the mouse pointer on the screen.

On the contrary, velocity control uses an additional integration step between the estimate input and control output. In Figure 28 this is indicated by the optional third integrator component of the post-processing. In velocity control, if the user keeps the hand relaxed $(\hat{v}(t)=0$ and $\hat{\varphi}(t)=0)$, the output of the post-processing is constant (i.e. arrow in VEP stays in the current position). But if the user performs a activates muscles $(\hat{v}(t) \neq 0$ and $\hat{\varphi}(t) \neq 0)$, the output of the post-processing is changing (i.e. the arrow in the VEP moves). This is equal to using a joystick: In relaxed position the curser stays in place. While pushing the joystick in any direction, the curser moves in the corresponding direction as long as the joystick is activated.

At this point it should be noted that the velocity control is the common control mode of both the industrial state of the art and the pattern recognition systems [1], [109], [110]. 
Finally, the output of the post-processing $\left(v_{\text {out }}\right.$ and $\left.\varphi_{\text {out }}\right)$ is used as the control signal send to the VEP or a prosthetic device connected. 


\section{Experimental studies on the new myocontrol scheme and results}

Two studies were performed to evaluate the result of this development towards a new control system for prosthetic hands. The first study focused on the gain in controllability, provided by the introduction of the ACAR filter into the developed control system.

The second study evaluated the allover performance of the control system including both intact-limb subjects as well as amputee subjects.

The presented studies were approved by the local ethics committee of the Universitätsmedizin Göttingen ${ }^{6}$. All subjects read and signed the informed consent.

\subsection{Study 1: Impact of the ACAR filter on the Online Regression Control System}

\subsubsection{Methods}

To evaluate and quantify the impact of the ACAR filter, the Performance assessment task with the Virtual Evaluation Paradigm has been used. Four intact-limb subjects S1-S4 (male, age range 25-39) participated in this study. All subjects were fitted with the 16 electrode setup as described in section 2.3.2.1.

After the subject preparation, calibration data for the wrist flexion+extension (DOF 1) and pronation+supination (DOF 2 ) was acquired using the Guided calibration mode.

\footnotetext{
${ }^{6}$ Application no 8/2/11, "Joint EEG, surface EMG and Intramuscular EMG Recordings for the Validation of Motor Control Muscle Synergy Model”, approved May $11^{\text {th }} 2011$.
} 
Next, the control system was calibrated in three different pre-processing configurations with the previously recorded raw EMG data. The three configurations differed by the ACAR filter stage of the pre-processing (see section 2.3.2.2.1). In the ACAR configuration and the CAR [51] configuration, the respective filter was used to improve the selectivity of the EMG channels. In the RAW configuration no filter was used at all and the EMG linear envelope was calculated from the EMG signal as acquired by the EMG amplifier. The sequence of the configurations was randomized among the subjects. After every calibration and while maintaining the pre-processing configuration, the subject was asked to hit 20 standardized targets, distributed in the control range, that all required simultaneous and proportional control to be accomplished (target type 3, $T_{\max }=20 \mathrm{~s}, T_{\text {dwell }}=300 \mathrm{~ms}, d_{\text {circle }}=16 \mathrm{dp}$ ).

The time to reach the targets as well as a completion rate was recorded and used as performance metrics. The results were statistically tested using two-factor ANOVAs, with the pre-processing method and the subject as factors. A significance level of $\alpha=0.05$ was used for all tests.

\subsubsection{Results}

The resulting performance metrics of the online control task for the four subjects are presented in Figure 29. For the subjects S1 and S2, the completion rates increased equally using CAR or ACAR in comparison to the unfiltered EMG signal. For subject S4 the completion rate was the highest using ACAR, while using the unprocessed EMG signal only 6 out of 20 targets were completed. In fact, the controllability of the online paradigm for this subject in the RAW configuration was very poor. For the very experienced subject S3 all three preprocessing methods yielded $100 \%$. However, for all subjects, the ACAR preprocessing resulted in the most stable control experience. 

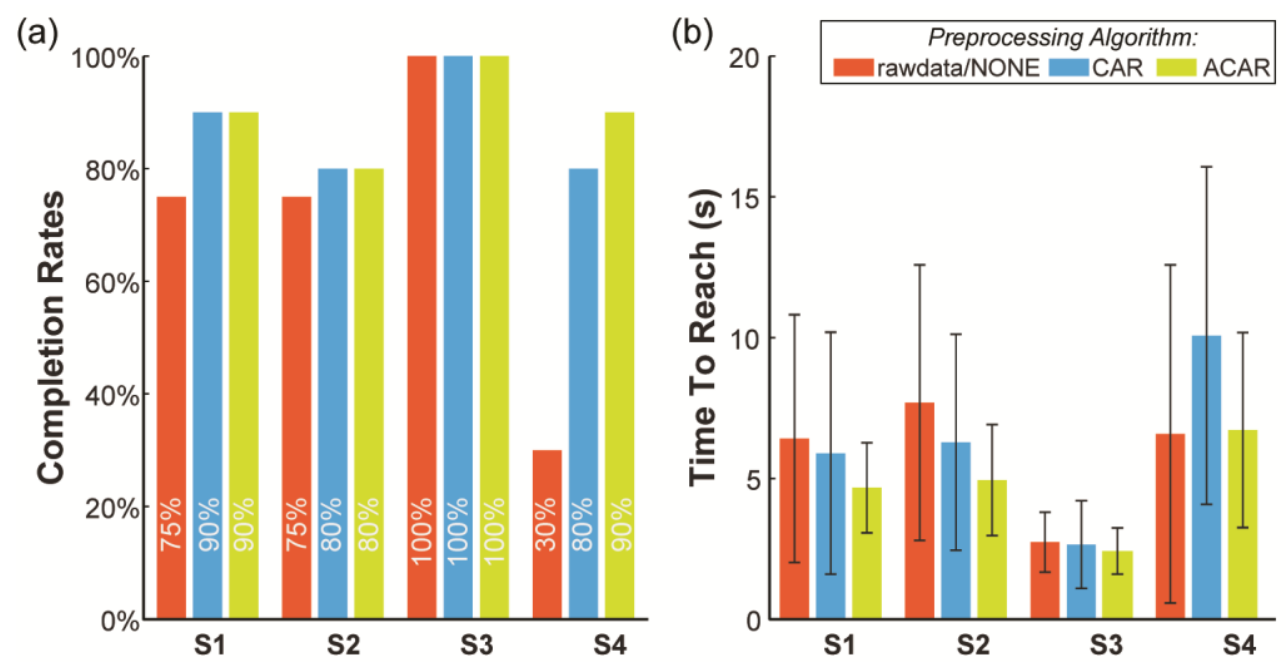

Figure 29: Acquired metric data of the online regression myocontrol using NMF. The left plot (a) shows the completion rate for the $\mathbf{2 0}$ targets of the four subjects using the three different EMG preprocessing methods in randomized order. The corresponding mean time to reach the taraets and standard deviation is aiven in the riaht plot (b).

This controllability is represented by the time to reach the target (Figure 29b). For all subjects the mean time to reach the target as well as the standard deviation was the lowest for the ACAR preprocessing, followed by the CAR algorithm.

For subject S4, the unfiltered EMG signal yielded a similar mean time to reach as for ACAR, while the standard deviation was about double. However, since this subject was able to complete only $30 \%$ of the targets, specifically those that were the closest from the initial position and therefore easier to reach, the unfiltered EMG (RAW configuration) obviously performed poorer than both CAR and ACAR.

The statistical analysis of the time to reach on all data acquired indicated an evident significance $(p<0.05)$.

\subsubsection{Study discussion}

The evaluation of the proposed ACAR algorithm in an online performance task with 20 targets resulted in a significant increase of controllability. In this evaluation, all subjects performed best with the ACAR as part of the preprocessing chain. As the ACAR filter increases the separability while reducing the noise, the subjects 
experienced a more smooth control of the arrow, resulting in a faster and more reliable completion of the tasks.

\subsection{Study 2: Evaluation of the Online Regression Control System}

\subsubsection{Methods}

The second study was designed to evaluate the performance of the online regression control system for different subjects in position mode. For this study no only able-body subjects were included in the study, but also subjects with limb deficiencies, such as amputations or congenital malformations.

In total, 11 subjects participated in the study, 3 subjects with limb deficiencies and 8 intact-limb control subjects. Subjects D2 and D3 used a myoelectric hand prosthesis (Otto Bock MyoHand) on a daily basis, while subject D1 had never used a hand prosthesis before. A detailed list of all subjects is presented in Table 3. For this study, the subjects were fitted with the 16 electrodes setup as described in section

Table 3: Summary of the subjects who participated in Study 2

ID Age Gender Subject Condition

\begin{tabular}{lccl}
\hline D1 & 38 & M & $\begin{array}{l}\text { congenital malformation, right side, } \\
\text { hand is missing from the level of the wrist }\end{array}$ \\
\hline D2 & 72 & M & $\begin{array}{l}\text { amputation 30 years ago, left side, } \\
\text { transradial short stump (approx. } 8 \mathrm{~cm})\end{array}$ \\
\hline D3 & 46 & M & $\begin{array}{l}\text { amputation 3 years ago, left side, } \\
\text { transradial long stump (approx. 17 cm) }\end{array}$ \\
\hline C1 & 56 & F & intact-limb \\
\hline C2 & 30 & F & intact-limb \\
\hline C3 & 33 & M & intact-limb \\
\hline C4 & 27 & M & intact-limb \\
\hline C5 & 31 & M & intact-limb \\
\hline C6 & 29 & M & intact-limb \\
\hline C7 & 35 & M & intact-limb \\
\hline C8 & 28 & M & Intact-limb \\
\hline
\end{tabular}




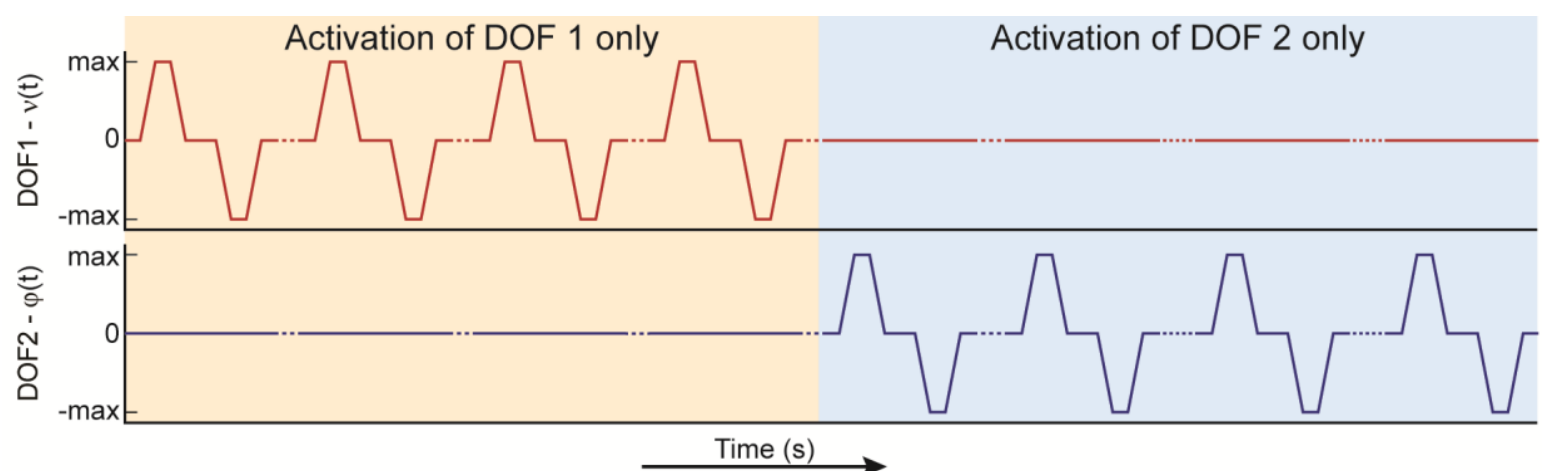

Figure 30: Prompt sequence used for the calibration phase of study 2. The subject was presented with four dynamic activations within the range of motion for both DOF. Each trial was triggered manually by the experimenter. Therefore, the time between two repetitions (dotted sections) varied depending on the subject's preference.

2.3.2.1, and were asked to seat comfortably with their arm extended, approximately 180 degrees elbow extension.

Each subject performed the same study protocol. In the first phase of the protocol, EMG data for the calibration of the regression system was recorded using the Guided calibration mode. The subject was asked to perform dynamic activations within the maximum range of motion for DOF1 (wrist flexion/extension) and DOF2 (wrist pronation/supination). The activations of each DOF were repeated four times and were triggered manually by the experimenter. The prompt sequence for $v(t)$ and $\varphi(t)$ presented to the subjects is given in Figure 30.

Next, this EMG sample data was processed and the inverse synergy matrix, the permutation matrix and the scaling factors were determined, as described in section

\subsubsection{4.}

Once the online control system was calibrated, the subject had approx. 5 minutes to try to control the arrow. For the FIR filter of the post-processing during online control, the parameters were chosen as $b_{0}=b_{1}=b_{2}=b_{3}=b_{4}=\frac{1}{5}$. Considering the update rate of $40 \mathrm{~ms}$, this implied that the last $200 \mathrm{~ms}$ of newly acquired EMG signals were taken 
into account for the generation of an output estimate. The scaling factors $\left[\begin{array}{llll}\alpha_{\mathrm{DOF} 1}^{+} & \alpha_{\mathrm{DOF} 1}^{-} & \alpha_{\mathrm{DOF} 2}^{+} & \alpha_{\mathrm{DOF} 2}^{-}\end{array}\right]$were manually adjusted by the experimenter, to adapt the available range of motion of the arrow.

Afterwards, the second phase of the study protocol started. In this phase, the control performance was evaluated using the Performance assessment task with the parameters $T_{\max }=20 \mathrm{~s}, T_{\text {dwell }}=300 \mathrm{~ms}$. The diameter of the circle was chosen as $d_{\text {circle }}=16 \mathrm{dp}$, because in this case the size of one circle is approx. $1 \%$ of the entire working space $\left(A_{\text {Workspace }}=360 \mathrm{dp} \times 60 \mathrm{dp}\right)$.

Each subject was presented with a standardized run, containing one series of 10 targets for each target type (1,2 and 3$)$ in the subject's range of motion. The series for target type 1 and target type 2 requiring only one DOF were presented first, but the order of those two series was permutated randomly among the subjects. In this manner, the subject was able to accommodate to the system and learn to control the two degrees of freedom separately. Finally, the third series for target type 3 requiring both DOF was presented to the subjects.

Once all data was acquired, the following performance metrics introduced in chapter 2.2.6 were extracted:
i) the task completion rate $\alpha[\%]$
ii) task completion time $t_{c}[s]$,
iii) path efficiency $\Gamma[\%]$,
iv) overshoots $k$
v) $\quad$ speed $v[\mathrm{dp} / s]$. 


\subsubsection{Results}

The results for task completion rate, task completion time, and path efficiency are presented in Figure 31. The results are reported for each subject separately and all metrics were calculated for the whole run as well as for disaggregated by the three target types. For the completion time and path efficiency, the means and standard deviations were calculated including only the successfully completed tasks.

Looking at the task completion rate, the subjects with limb deficiencies D1 and D3
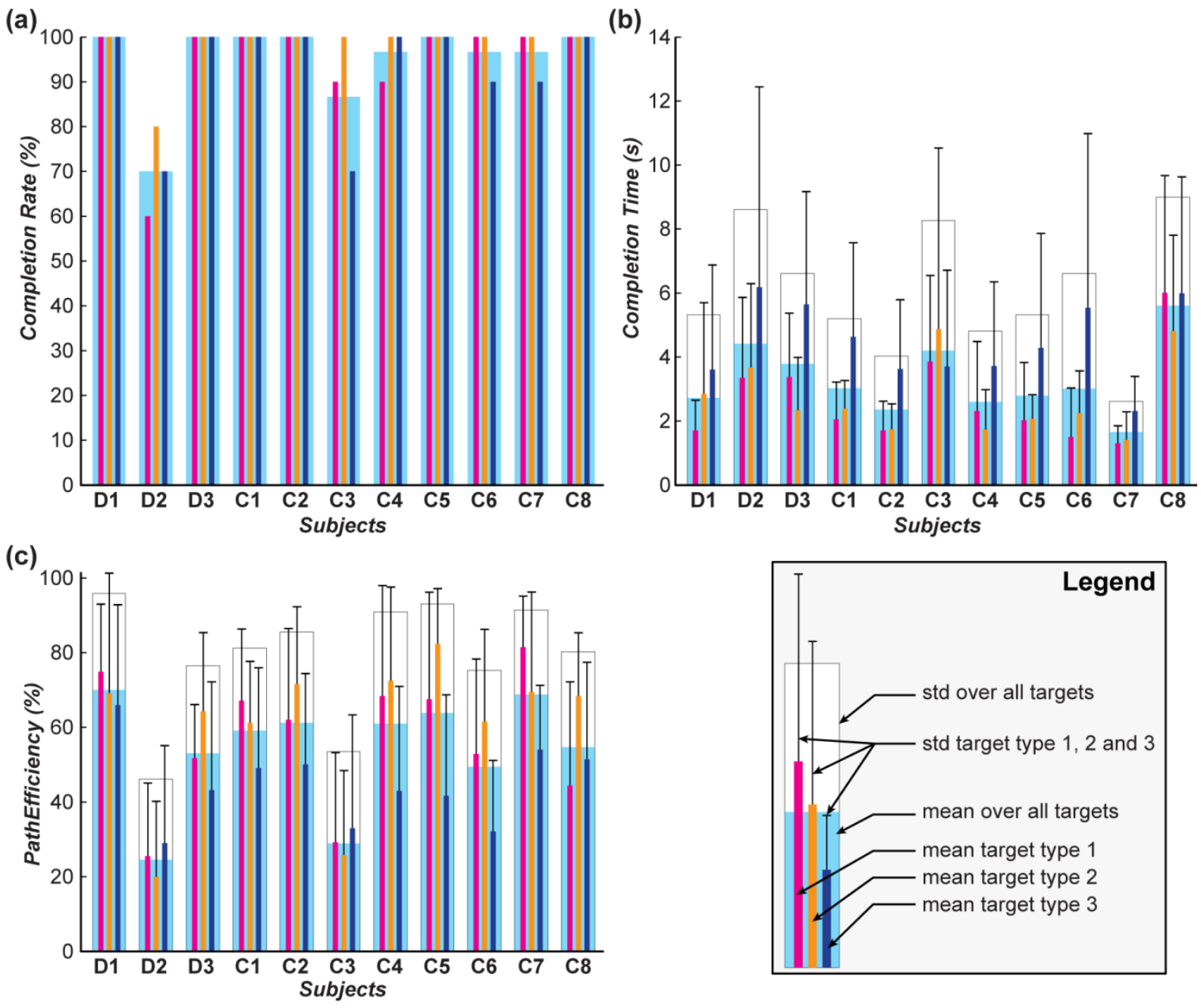

Figure 31: Metrics for all subjects acquired with the Performance Assessment Task. For each subject, the mean and standard deviation for each metric are reported for the full run (wide light blue bars) as well as for each target type separately (thin color bars magenta, orange and dark blue on top of the light blue bars). Note the legend provided in the lower right for the detailed description of the plot. The completion rate (a) reports the number of targets that were successfully completed per subject. The mean and standard deviation for the completion time (b) and path efficiency (c) are calculated on the basis of the successfully completed targets only. 
were able to complete $100 \%$ of the targets while D2 completed only $70 \%$ of all targets. In case of the eight control subjects, four subjects were able to accomplish $100 \%$ of the targets, three subjects missed only one of the 30 presented targets and only subject C3 completed less than $90 \%$ of all targets.

Similar results are reflected in the metrics for completion time and path efficiency. Subject D2 and C3 required more time than all other subjects and also had worse path efficiency than all other subjects. Subject C8 also had longer completion times. However, during the study it was observed that subject C8 chose a particular strategy to control the arrow slowly, but steady and precise in comparison to D2 and C3 who did not perform well.

Generally, the completion time for the series of target type 3 was $20-100 \%$ longer for most of the subjects, except for subjects C3 and C8. This is expected because these targets are more demanding. The user had to hit these targets controlling the arrow simultaneously and proportionally. Additionally, for the same reason the path efficiency was generally worse for the targets of type 3. On the contrary, the
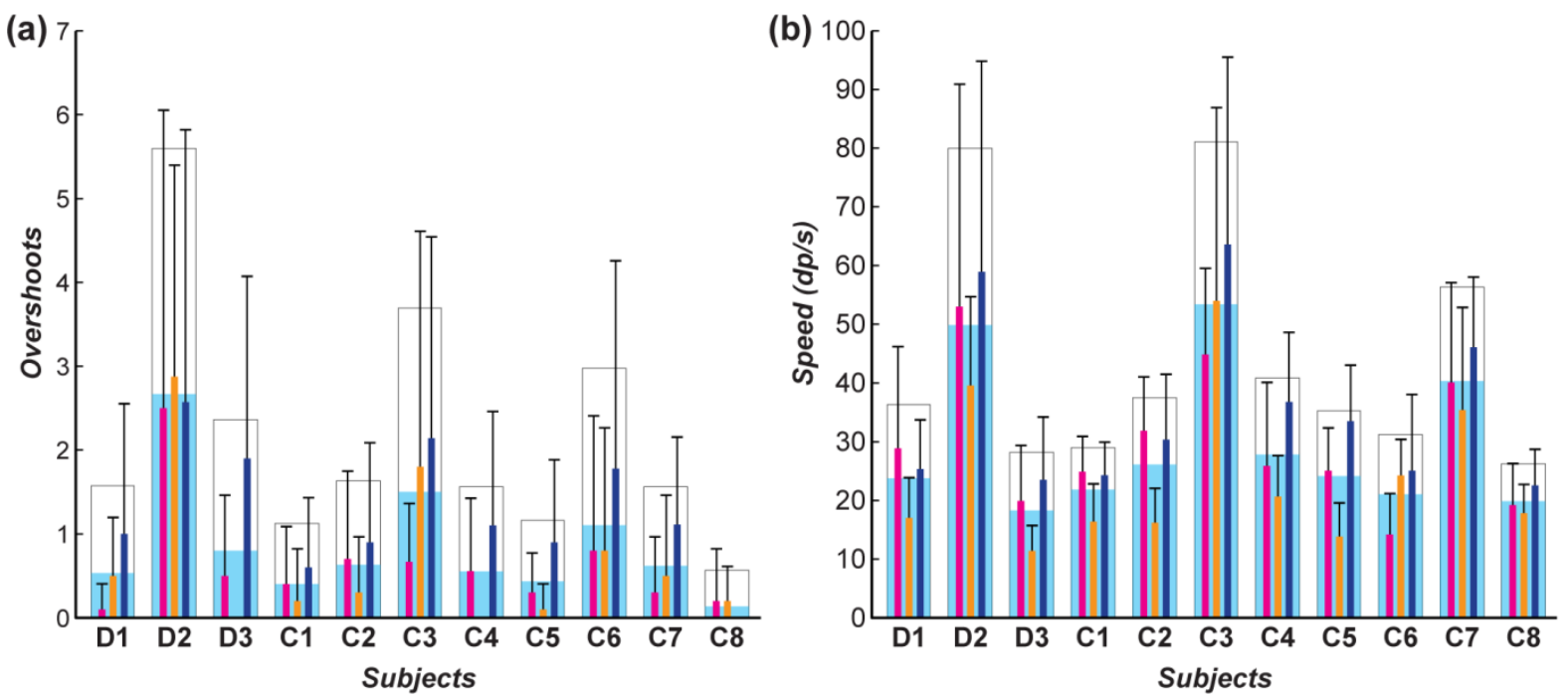

Figure 32: The metric overshoots (a) counts the number of times that the subject positioned the tip of the arrow onto the target circle, but did not manage to remain on the target for the required dwelling time. The speed (b) measures the average moving speed of the arrow's tip. 
standard deviations of the path efficiency were very similar among target types, while the standard deviations of the completion time for targets of type 3 were generally larger.

Besides the previous three metrics, the metrics for overshoots and speed were also calculated and are reported in Figure 32. In most cases the number of overshoots was higher for the targets of type 3. Interestingly, subject C8 had very few overshoots and no overshoots at all for target type 3.This might be explained by his slow but stable strategy to control the arrow. Subjects D2 and C3 performed worse again; they had the highest number of overshoots. Subject D2 had similar overshoots for all target types. He was able to hit all target types, but needed on average more than 2 attempts to hit the target, before being able to remain there for the required dwelling time for a successful task completion.

Considering the speed metric, subjects D2 and C3 again performed different than the rest of the subjects. The average speed for these two subjects was about twice the average speed of the other subjects. Consistently, the standard deviations were also larger. Interestingly, the average speed of subject C8 just was minimally slower than the speed of most of the other subjects and with a very low variation. All other subjects had similar results for both the mean and the standard deviation of the speed metric.

In order to investigate the relation between the completion time as the simplest metric and the other three target based metrics, the Pearson correlation coefficient $\rho$ and the corresponding statistically significant levels $p$ were obtained from the pooled measures of all subjects (Table 4). The correlation was calculated for the whole run as well as separately for the three different target types in order to 
Table 4: Selected correlations between the performance metrics

\begin{tabular}{|c|c|c|c|c|c|}
\hline & & Target Type 1 & Target Type 2 & Target Type 3 & Full Run \\
\hline \multirow{2}{*}{$\begin{array}{l}\text { Completion Time } \alpha \\
\text { Path Efficiency } \Gamma\end{array}$} & $\rho$ & -0.61 & -0.53 & -0.64 & -0.61 \\
\hline & $p$ & $7 e-12$ & $3 e-09$ & $4 e-13$ & $2 e-33$ \\
\hline \multirow{2}{*}{$\begin{array}{c}\text { Completion Time } \\
\text { Overshoots } k\end{array}$} & $\rho$ & 0.35 & 0.62 & 0.60 & 0.57 \\
\hline & $p$ & 0.0003 & $1 \mathrm{e}-12$ & $3 e-11$ & $3 e-28$ \\
\hline \multirow{2}{*}{$\begin{array}{c}\text { Completion Time } \alpha \\
\text { Speed } v\end{array}$} & $\rho$ & 0.23 & 0.21 & 0.28 & 0.28 \\
\hline & $p$ & 0.02 & 0.032 & 0.004 & $5 e-07$ \\
\hline
\end{tabular}

compare the diffepences between the target types. The completion time was significantly and markedly correlated with the path efficiency (negative correlation) and the overshoots (positive correlation). Additionally, the completion time correlated weakly and significantly with the speed (compare with Figure 31 ).

These significant correlations indicate the consistency of the choice of these four metrics for the assessment of the successful task completion. Furthermore, the correlations were marked, but did not reach a $100 \%$ correlation indicating that these metrics should be employed together because they underline different aspects of the task completion.

Finally, a statistical analysis of the performance metrics has been performed to find the differences between the subject with limb deficiencies and the intact-limb subjects as well as the impact of the target type.

The non-parametric statistical test Kruskal-Wallis test [111] was used because the distributions of the performance metrics were not Gaussian. To assess the impact of the subject (limb deficiency vs. intact-limb), the subjects condition was used as a grouping factor. Secondly, the same test was performed using the target type $(1,2,3)$ as grouping factor. The results of these tests are given in Table 
Table 5: $p$-Values of the Kruskal-Wallis tests for the five performance metrics

\begin{tabular}{lccccc}
\multicolumn{1}{c}{ Factor } & $\begin{array}{c}\text { Completion } \\
\text { Rate }\end{array}$ & $\begin{array}{c}\text { Completion } \\
\text { Time }\end{array}$ & $\begin{array}{c}\text { Path } \\
\text { Efficiency }\end{array}$ & Overshoots & Speed \\
\hline \hline $\begin{array}{l}\text { Subject Condition } \\
\text { (limb deficiency/ intact-limb) }\end{array}$ & 1 & 0.22 & 0.25 & 0.17 & 0.27 \\
\hline $\begin{array}{l}\text { Target Type } \\
(1,2,3)\end{array}$ & 0.34 & $10^{-6}$ & $2 * 10^{-4}$ & $4 * 10^{-4}$ & $10^{-6}$ \\
\hline
\end{tabular}

5. The subject condition did not have a significant influence on any of the five performance metrics. This indicates that amputees could use the systems as well as intact-limb subjects. The target type had significant influence on all performance metrics except for the completion rate. This indicates that all subjects could complete all given tasks, even though the tasks with target type 3 were more demanding.

\subsubsection{Study discussion}

In this study the use of an online control system for simultaneous and proportional has been demonstrated and evaluated using a target-based assessment task. By including also subjects with limb deficiencies, the transferability of the results to the possible target group of the developed algorithm has been ensured.

Generally, all subjects were able to complete the majority of the provided tasks for the three target types. The statistical analysis for the performance metrics proved no significant differences between the results of the subjects with limb deficiencies and the intact-limb control subjects. As an important finding, these results indicate that the Online Regression Control System allows a similar control experience for both subjects groups. 


\section{Discussion}

In the previous chapters three key elements in the field of myoelectric control for upper limb prostheses have been presented and evaluated in human studies. The first is the ACAR filter that has been developed as a new pre-processing stage for monopolar EMG signals. By introducing an adaptive channel selection algorithm for the calculation of a time-varying common average reference, an increase in selectivity and a reduction of the common noise have been achieved. By applying the adaptive filter to the surface EMG data recorded from different subjects, this improvement in signal quality has also been quantified and an optimal parameter set has been identified (section 2.1.3.1).

Besides the quantitative evaluation of the signal quality, the ACAR filter was tested with EMG data using classical offline pattern recognition for myoelectric control, too. Here again, a significant improvement of the inter-class distribution of the classes in the feature space was demonstrated.

However, the most important aim of this development process was to improve the signal conditioning for the estimation of the muscle activation in the online regression system. This improvement was confirmed by the results of the study in section 3.1, including four subjects. The direct comparison of the control performance using different pre-processing stages resulted in a significant gain provided by the ACAR filtering. Consequently, the ACAR was included as a key solution in the signal conditioning of the Online Regression Control System. 
Another important advantage of the ACAR filter is the computational simplicity and the fact that no training is required. In the proposed configuration, each processing step is based on the calculation of the sum over absolutes, a sorting step and the calculation of the sample-wise mean to be subtracted from the input data. All these processing steps can be performed on basic microcontrollers or even embedded in hardware. And as no training is required, the algorithm runs out of the box.

While the discussed applications are focusing mainly on myocontrol, the ACAR filter also has potential impact in other EMG applications. For instance, the filter has also been used on recordings of High-Density EMG signals from TMR subjects with 384+ channels [112], [113]. In this case, the filter was used to enhance the spatial resolution of the EMG activation during online visualizations and permitted a direct and distinct visual feedback to the subject.

The second key element of this project is the Virtual Evaluation Paradigm, including Performance assessment task and performance metrics. While in the scientific community the methods and performance metrics for offline analysis are clearly defined, the online assessment has received a lower attention in the past [21], [23], [45], [47]. Therefore, the result of this work presents a simple but powerful and reliable methodology to assess the online performance of systems for simultaneous and proportional control.

The developed paradigm provides a simple and intuitive 2D-interface that can be used on a standard computer monitor. In this manner, no additional hardware such as head mounted displays or 3D monitors are necessary, which can also be challenging for the subject [76], [77], [114]. None of the subjects in the two studies presented in chapter 3 had difficulties in understanding and using the paradigm, 
although subjects D2 and D3 did not own or use a computer and therefore where not used to graphical user interfaces. Consequently, due to its intuitiveness, this paradigm can also be used in clinical practice with subjects of different technical experiences.

For the Performance assessment task, the studies have also shown that a targetbased evaluation system is encouraging for the subjects. Especially the audio feedback was appreciated by the subject. Not only were the subjects motivated during the study, but also asked to keep on playing with the target hitting task afterwards. By modifying the parameters of target size, dwelling time and timeout, the difficulty can be adjusted for the requirements of each study. The quantitative performance metrics provided by the paradigm allow a comparison either between subjects or between different control systems. The task completion rate, task completion time and path efficiency are intuitive and direct metrics. Specifically, the task completion rate gives an overall estimate of the performance achieved and is also easy to understand for the subject as a direct feedback. The task completion time encodes the responsiveness of the control system as well as the specific performance of the subject. However, the task completion time also is connected to each task, as targets more distant from the center position will require a longer time to reach. On the contrary, the path efficiency is generally unrelated to each task, as it is normalized to the optimal path. Nevertheless, in case of the target type 3 , the maximum path efficiency of $100 \%$ can only be achieved by using simultaneous control, which in turn is not available for all myocontrol system [13], [21], [109]. Therefore, the presented evaluation paradigm with its metrics has the advantage to actually account for the future requirement of prosthesis control systems of simultaneous and proportional control. 
Considering the additional metrics overshoots and speed, they can be considered as more abstract. An increased value for overshoots indicates a lack of fine control. The targets can only be completed after multiple attempts or corrections, implying also a decrease in path efficiency. The speed on the other hand depends both on the completion time and the path trajectory measured, thus all three metrics have to be considered together. A high speed together with a short task completion time and high path efficiency implies a fast and efficient control performance. On the contrary, a high speed together with a long task completion time implies that the trajectory was not optimal and probably included loops. In summary, due to the correlations of the metrics, they are complementing each other and form a sound basis for the evaluation of myocontrol systems.

The Virtual Evaluation Paradigm has been implemented within a more complex software environment [79], providing a toolset for signal processing, pattern recognition and regression systems. As a result, the developed paradigm has already been used in multiple studies including scientific collaborators and the results have been submitted or published in peer-reviewed journals or conferences [61], [65], [82]-[85], [89]. This underlines the importance and acceptance of this paradigm in the myocontrol community.

A short video demonstrating the use of the Performance Assessment Task by an amputee subjects is available at: $\underline{h t t p: / / y o u t u . b e / f X s P w b L s X y E}$

The third and final key component of this work is the Online Regression Control System. Starting from the work done by Jiang et al in 2009 to investigate the force estimation from muscle signals in offline studies using a factorization approach [14], a practicable online myocontrol system has been developed. Despite the fact that 
factorization methods and especially the NMF approach have already been proposed by the scientific community in the past as a potential solution for intuitive prostheses control, only the pre- and post-processing methods developed within this PhD project finally closed the existing gap towards a viable online estimation of hand kinematics. With the new signal conditioning stage and the post-processing to stabilize the output, a completely new system for the simultaneous and proportional control of hand prostheses has been developed. By designing the resulting system to run online and in real-time on standard computer hardware, it was possible to evaluate the applicability of the developed algorithms.

As mentioned before, also the implementation of the control system has been realized as a component of a larger software environment developed at the beginning of this project [79]. This ensures the interconnectivity with different hardware and a stable software basis. The use of the algorithm by different subjects has been shown in a study including 3 subjects with limb-deficiencies and 8 intactlimb control subjects (section 3.2). All of the subjects were able to control the two degrees of freedom simultaneously and proportionally, which has been evaluated using the Performance assessment task. The statistical analysis of the acquired performance metrics pointed out that the ability to use the control algorithm was independent from the subject condition.

Due to the short and easy calibration phase, the presented control system can be used within 5 minutes after placing the electrodes. In comparison to classical pattern recognition methods, such a short preparation time is reasonable for a prosthetic user, for instance to calibrate the prosthetic hand in the morning after donning. For 
such a use case, the prosthesis can even be used to provides the cue during the short calibration phase [44], [106].

While the quantitative analysis of the control system proof the usability, also the real application of the control system for hand prostheses has been tested in a use case. For this, the output of the post-processing system has been connected to a virtual prosthetic hand as well as a real Michelangelo Hand provided by Ottobock. However, at the current time the virtual hand and real prosthetic hand do not provide performance metrics that can be used in a study. To provide the reader an impression about the results, two videos have been produced and are available online:

- Michelangelo Hand: http://youtu.be/fjKi8NXZoi4

- Virtual Hand Prosthesis: http://youtu.be/XbHKGhlfCtU

While this thesis project targeted the use of myoelectric control in hand prosthetics, the use of the developed algorithm is not limited to this. Instead, it can also be used as a new kind of human interface device to interact with computers or smartphone. An example is the use of the generated control signal to control the mouse pointer on a computer. A similar application has already been tested and presented in [115].

As a final conclusion, a myocontrol system with an adaptive pre-processing algorithm has been developed, capable of controlling two degrees of freedom simultaneously and proportionally. Furthermore, the Virtual Evaluation Paradigm has been designed and implemented as a powerful tool to acquire calibration data, but also to evaluate the performance of myocontrol systems. The use of this paradigm 
as a scientific tool in multiple studies underlines the impact for the research area of neurorehabilitation. 


\section{$\underline{\text { References }}$}

[1] H. G. Näder and L. Milde, Otto Bock Prothesen-Kompendium: Prothesen für die obere Extremität, 2nd ed. Duderstadt: Otto Bock HealthCare GmbH, 2011.

[2] D. Childress, "Historical aspects of powered limb prostheses," Clin. prosthetics Orthot., 1985.

[3] R. Scott, "Myoelectric Control Of Prostheses: A Brief History," Proc. 1992 MyoElectric Control. Prosthetics Symp. Fredericton, New Brunswick, Canada, 1992.

[4] "In memoriam Reinhold Reiter 17 November 1920-24 September 1998," Int. J. Biometeorol., vol. 43, no. 2, pp. 96-98, Oct. 1999.

[5] A. E. Kobrinskiy, "Utilization of Bio-Currents for Control Purposes," 1959.

[6] P. M. Pilarski, M. R. Dawson, T. Degris, J. P. Carey, K. M. Chan, J. S. Hebert, and R. S. Sutton, "Adaptive artificial limbs: a real-time approach to prediction and anticipation," IEEE Robot. Autom. Mag., vol. 20, no. 1, pp. 53-64, Mar. 2013.

[7] A. E. Kobrinskiy, "Bioelectrical Control of Prosthetic Devices," Her. Acad. Sci., no. 30 , pp. 58-61, 1960.

[8] B. Popov, "The Bio-Electrically Controlled Prosthesis," J. Bone Joint Surg. Br., vol. 47, no. 3, pp. 421-4, Aug. 1965.

[9] C. K. Battye, A. Nightingale, and J. Whillis, "The use of myo-electric currents in the operation of prostheses.," J. Bone Joint Surg. Br., vol. 37-B, no. 3, pp. 506-10, Aug. 1955.

[10] A. Bottomley, P. Styles, P. Jilbert, J. Birtill, and J. Truscott, "Prosthetic hand with improved control system for activation by electromyogram signals," US Pat. 3,418,662, 1968.

[11] R. N. Scott, "Myoelectric Control of Prostheses and Orthoses," Bull. Prosthet. Res., vol. 7, p. 93, 1967.

[12] T. W. Williams III, "Controlling powered upper extremity prostheses now and in the future," 2005.

[13] P. Parker, K. Englehart, and B. Hudgins, "Myoelectric signal processing for control of powered limb prostheses.," J. Electromyogr. Kinesiol., vol. 16, no. 6, pp. 541-8, Dec. 2006. 
[14] N. Jiang, K. B. Englehart, and P. a Parker, "Extracting simultaneous and proportional neural control information for multiple-DOF prostheses from the surface electromyographic signal.," IEEE Trans. Biomed. Eng., vol. 56, no. 4, pp. 1070-80, Apr. 2009.

[15] J. L. G. Nielsen, S. Holmgaard, N. Jiang, K. Englehart, D. Farina, and P. Parker, "Enhanced EMG signal processing for simultaneous and proportional myoelectric control.," Conf. Proc. IEEE Eng. Med. Biol. Soc., vol. 2009, pp. 4335-8, Jan. 2009.

[16] S. Muceli and D. Farina, "Simultaneous and proportional estimation of hand kinematics from EMG during mirrored movements at multiple degrees-offreedom.," IEEE Trans. Neural Syst. Rehabil. Eng., vol. 20, no. 3, pp. 371-8, May 2012.

[17] P. Herberts and C. Almström, "Hand prosthesis control via myoelectric patterns," Acta ..., pp. 389-409, 1973.

[18] D. Graupe and W. K. Cline, "Functional Separation of EMG Signals via ARMA Identification Methods for Prosthesis Control Purposes," leee Transactions On Systems Man And Cybernetics, vol. SMC-5, no. 2. IEEE, pp. 252-259, 1975.

[19] F. R. Finley and R. W. Wirta, "Myocoder studies of multiple myopotential response.," Arch. Phys. Med. Rehabil., vol. 48, no. 11, pp. 598-601, Nov. 1967.

[20] B. Hudgins, P. Parker, and R. N. Scott, "A new strategy for multifunction myoelectric control.," IEEE Trans. Biomed. Eng., vol. 40, no. 1, pp. 82-94, Jan. 1993.

[21] A. Fougner, O. Stavdahl, P. J. Kyberd, Y. G. Losier, and P. a Parker, "Control of upper limb prostheses: terminology and proportional myoelectric control-a review.," IEEE Trans. Neural Syst. Rehabil. Eng., vol. 20, no. 5, pp. 663-77, Sep. 2012.

[22] M. A. Oskoei and H. Hu, "Myoelectric control systems-A survey," Biomed. Signal Process. Control, vol. 2, no. 4, pp. 275-294, Oct. 2007.

[23] E. Scheme and K. Englehart, "Electromyogram pattern recognition for control of powered upper-limb prostheses: State of the art and challenges for clinical use," J. Rehabil. Res. Dev., vol. 48, no. 6, p. 643, 2011.

[24] K. Englehart and B. Hudgins, "A robust, real-time control scheme for multifunction myoelectric control.," IEEE Trans. Biomed. Eng., vol. 50, no. 7, pp. 848-54, Jul. 2003.

[25] A. M. Simon, K. Stern, and L. J. Hargrove, "A comparison of proportional control methods for pattern recognition control.," Conf. Proc. IEEE Eng. Med. Biol. Soc., vol. 2011, pp. 3354-7, Jan. 2011. 
[26] K. Englehart, B. Hudgins, and P. a Parker, "A wavelet-based continuous classification scheme for multifunction myoelectric control.," IEEE Trans. Biomed. Eng., vol. 48, no. 3, pp. 302-11, Mar. 2001.

[27] M. A. Oskoei and H. Hu, "Support vector machine-based classification scheme for myoelectric control applied to upper limb.," IEEE Trans. Biomed. Eng., vol. 55, no. 8, pp. 1956-65, Aug. 2008.

[28] E. Ceseracciu, M. Reggiani, Z. Sawacha, M. Sartori, F. Spolaor, C. Cobelli, and E. Pagello, "SVM classification of locomotion modes using surface electromyography for applications in rehabilitation robotics," 19th Int. Symp. Robot Hum. Interact. Commun., pp. 165-170, Sep. 2010.

[29] C. Castellini, E. Gruppioni, A. Davalli, and G. Sandini, "Fine detection of grasp force and posture by amputees via surface electromyography.," J. Physiol. Paris, vol. 103, no. 3-5, pp. 255-62.

[30] T. Lorrain, N. Jiang, and D. Farina, "Influence of the training set on the accuracy of surface EMG classification in dynamic contractions for the control of multifunction prostheses.," J. Neuroeng. Rehabil., vol. 8, no. 1, p. 25, Jan. 2011.

[31] F. V. G. Tenore, A. Ramos, A. Fahmy, S. Acharya, R. Etienne-Cummings, and N. V Thakor, "Decoding of individuated finger movements using surface electromyography.," IEEE Trans. Biomed. Eng., vol. 56, no. 5, pp. 1427-34, May 2009.

[32] Y. Huang, K. B. Englehart, B. Hudgins, and A. D. C. Chan, "A Gaussian mixture model based classification scheme for myoelectric control of powered upper limb prostheses.," IEEE Trans. Biomed. Eng., vol. 52, no. 11, pp. 180111, Nov. 2005.

[33] F. E. R. Mattioli, E. a Lamounier, A. Cardoso, A. B. Soares, and A. O. Andrade, "Classification of EMG signals using artificial neural networks for virtual hand prosthesis control.," Conf. Proc. IEEE Eng. Med. Biol. Soc., vol. 2011, pp. 7254-7, Jan. 2011.

[34] L. J. Hargrove, K. Englehart, and B. Hudgins, "A comparison of surface and intramuscular myoelectric signal classification.," IEEE Trans. Biomed. Eng., vol. 54, no. 5, pp. 847-53, May 2007.

[35] P. C. Doerschuk, D. E. Gustafson, and A. S. Willsky, "Upper extremity limb function discrimination using EMG signal analysis.," IEEE Trans. Biomed. Eng., vol. 30, no. 1, pp. 18-29, Jan. 1983.

[36] L. J. Hargrove, E. J. Scheme, K. B. Englehart, and B. S. Hudgins, "Multiple binary classifications via linear discriminant analysis for improved controllability of a powered prosthesis.," IEEE Trans. Neural Syst. Rehabil. Eng., vol. 18, no. 1, pp. 49-57, Feb. 2010. 
[37] N. Jiang, S. Dosen, K.-R. Muller, and D. Farina, "Myoelectric Control of Artificial Limbs-Is There a Need to Change Focus? [In the Spotlight]," IEEE Signal Process. Mag., vol. 29, no. 5, pp. 152-150, Sep. 2012.

[38] a J. Young, L. J. Hargrove, and T. a Kuiken, "Improving Myoelectric Pattern Recognition Robustness to Electrode Shift by Changing Interelectrode Distance and Electrode Configuration.," IEEE Trans. Biomed. Eng., no. c, pp. 1-8, Nov. 2011.

[39] L. Hargrove, K. Englehart, and B. Hudgins, "A training strategy to reduce classification degradation due to electrode displacements in pattern recognition based myoelectric control," Biomed. Signal Process. Control, vol. 3, no. 2, pp. 175-180, Apr. 2008.

[40] a Fougner, E. Scheme, a Chan, K. Englehart, and O. Stavdahl, "Resolving the Limb Position Effect in Myoelectric Pattern Recognition.," IEEE Trans. Neural Syst. Rehabil. Eng., vol. 19, no. 6, pp. 644-651, Aug. 2011.

[41] S. Amsuss, L. P. Paredes, N. Rudigkeit, B. Graimann, M. J. Herrmann, and D. Farina, "Long term stability of surface EMG pattern classification for prosthetic control.," Conf. Proc. IEEE Eng. Med. Biol. Soc., vol. 2013, pp. 3622-5, Jul. 2013.

[42] N. Ge, P. M. Goebel, S. Amsuess, L. Paredes, R. Pawlik, and D. Farina, "Evaluating Upper-Limb EMG-Prosthesis User Performance by Combining Psychometric Measures and Classification-Rates," in 6th International IEEE EMBS Conference on Neural Engineering, 2013, pp. 4-7.

[43] L. Hargrove, Y. Losier, B. Lock, K. Englehart, and B. Hudgins, "A real-time pattern recognition based myoelectric control usability study implemented in a virtual environment.," Conf. Proc. IEEE Eng. Med. Biol. Soc., vol. 2007, pp. 4842-5, Jan. 2007.

[44] B. Lock, A. Simon, K. Stubblefield, and L. Hargrove, "Prosthesis-guided training for practical use of pattern recognition control of prostheses," Training, pp. 1-4, 2011.

[45] A. M. Simon, L. J. Hargrove, B. a. Lock, and T. a. Kuiken, "Target Achievement Control Test: Evaluating real-time myoelectric pattern-recognition control of multifunctional upper-limb prostheses," J. Rehabil. Res. Dev., vol. 48, no. 6, p. 619, 2011.

[46] M. Hauschild, R. Davoodi, and G. E. Loeb, "A virtual reality environment for designing and fitting neural prosthetic limbs.," IEEE Trans. Neural Syst. Rehabil. Eng., vol. 15, no. 1, pp. 9-15, Mar. 2007.

[47] B. Lock, K. Englehart, and B. Hudgins, "Real-time myoelectric control in a virtual environment to relate usability vs. accuracy," MyoElectric Control. Symp., pp. 17-20, 2005. 
[48] S. Muceli, N. Jiang, and D. Farina, "Multichannel surface EMG based estimation of bilateral hand kinematics during movements at multiple degrees of freedom.," Conf. Proc. IEEE Eng. Med. Biol. Soc., vol. 2010, pp. 6066-9, Jan. 2010.

[49] J. L. G. Nielsen, S. Holmgaard, N. Jiang, K. B. Englehart, D. Farina, and P. a Parker, "Simultaneous and proportional force estimation for multifunction myoelectric prostheses using mirrored bilateral training.," IEEE Trans. Biomed. Eng., vol. 58, no. 3, pp. 681-8, Mar. 2011.

[50] H. Rehbaum and D. Farina, "Adaptive common average filtering for myocontrol applications," Med. Biol. Eng. Comput., vol. submitted, 2013.

[51] D. J. McFarland, L. M. McCane, S. V David, and J. R. Wolpaw, "Spatial filter selection for EEG-based communication," Electroencephalogr. Clin. Neurophysiol., vol. 103, no. 3, pp. 386-394, Sep. 1997.

[52] M. Zecca, S. Micera, M. C. Carrozza, and P. Dario, "Control of Multifunctional Prosthetic Hands by Processing the Electromyographic Signal," Crit. Rev. Biomed. Eng., vol. 30, no. 4-6, pp. 459-485, Jan. 2002.

[53] L. J. Hargrove, G. Li, K. B. Englehart, and B. S. Hudgins, "Principal components analysis preprocessing for improved classification accuracies in pattern-recognition-based myoelectric control.," IEEE Trans. Biomed. Eng., vol. 56, no. 5, pp. 1407-14, May 2009.

[54] J. M. Hahne, B. Graimann, and K.-R. Müller, "Spatial filtering for robust myoelectric control.," IEEE Trans. Biomed. Eng., vol. 59, no. 5, pp. 1436-43, May 2012.

[55] C. J. De Luca and R. Merletti, "Surface myoelectric signal cross-talk among muscles of the leg," Electroencephalogr. Clin. Neurophysiol., vol. 69, no. 6, pp. 568-575, Jun. 1988.

[56] T. J. Koh and M. D. Grabiner, "Evaluation of methods to minimize cross talk in surface electromyography.," J. Biomech., vol. 26 Suppl 1, pp. 151-7, Jan. 1993.

[57] H. Broman, G. Bilotto, and C. J. De Luca, "A note on the noninvasive estimation of muscle fiber conduction velocity.," IEEE Trans. Biomed. Eng., vol. 32, no. 5, pp. 341-4, May 1985.

[58] J. Van Vugt and J. Van Dijk, "A convenient method to reduce crosstalk in surface EMG," Clin. Neurophysiol., vol. 112, no. 2000, pp. 583-592, 2001.

[59] C. Disselhorst-Klug, J. Silny, and G. Rau, "Improvement of spatial resolution in surface-EMG: a theoretical and experimental comparison of different spatial filters.," IEEE Trans. Biomed. Eng., vol. 44, no. 7, pp. 567-74, Jul. 1997. 
[60] D. T. Mewett, H. Nazeran, and K. J. Reynolds, "Removing power line noise from recorded EMG," in 2001 Conference Proceedings of the 23rd Annual International Conference of the IEEE Engineering in Medicine and Biology Society, 2001, vol. 3, pp. 2190-2193.

[61] J. M. Hahne, H. Rehbaum, F. Biessmann, F. C. Meinecke, K.-R. Muller, N. Jiang, D. Farina, and L. C. Parra, "Simultaneous and proportional control of 2D wrist movements with myoelectric signals," in 2012 IEEE International Workshop on Machine Learning for Signal Processing, 2012, pp. 1-6.

[62] P. Zhou, B. Lock, and T. a Kuiken, "Real time ECG artifact removal for myoelectric prosthesis control.," Physiol. Meas., vol. 28, no. 4, pp. 397-413, Apr. 2007.

[63] M. V Kinder, E. S. van Waalwijk van Doorn, E. D. Gommer, and R. A. Janknegt, "A non-invasive method for bladder electromyography in humans.," Arch. Physiol. Biochem., vol. 106, no. 1, pp. 2-11, Feb. 1998.

[64] H. Ramoser, J. Müller-Gerking, and G. Pfurtscheller, "Optimal spatial filtering of single trial EEG during imagined hand movement.," IEEE Trans. Rehabil. Eng., vol. 8, no. 4, pp. 441-6, Dec. 2000.

[65] H. Rehbaum, N. Jiang, L. Paredes, S. Amsuess, B. Graimann, and D. Farina, "Real time simultaneous and proportional control of multiple degrees of freedom from surface EMG: Preliminary results on subjects with limb deficiency.," Conf. Proc. IEEE Eng. Med. Biol. Soc., vol. 2012, pp. 1346-9, Jan. 2012.

[66] N. E. Bunderson and T. a Kuiken, "Quantification of feature space changes with experience during electromyogram pattern recognition control.," IEEE Trans. Neural Syst. Rehabil. Eng., vol. 20, no. 3, pp. 239-46, May 2012.

[67] G. R. Naik and D. K. Kumar, "Identification of hand and finger movements using multi run ICA of surface electromyogram.," J. Med. Syst., vol. 36, no. 2, pp. 841-51, Apr. 2012.

[68] L. Smith, L. Hargrove, and B. Lock, "Determining the optimal window length for pattern recognition-based myoelectric control: Balancing the competing effects of classification error and controller delay," Neural Syst., vol. 19, no. 2, pp. 186-92, Apr. 2011.

[69] J. W. Sensinger, B. a Lock, and T. a Kuiken, "Adaptive pattern recognition of myoelectric signals: exploration of conceptual framework and practical algorithms.," IEEE Trans. Neural Syst. Rehabil. Eng., vol. 17, no. 3, pp. 270-8, Jun. 2009.

[70] R. Kato, T. Fujita, H. Yokoi, and T. Arai, "Adaptable EMG Prosthetic Hand using On-line Learning Method -Investigation of Mutual Adaptation between Human and Adaptable Machine," in ROMAN 2006 - The 15th IEEE 
International Symposium on Robot and Human Interactive Communication, 2006, pp. 599-604.

[71] C. M. Light, P. H. Chappell, and P. J. Kyberd, "Establishing a standardized clinical assessment tool of pathologic and prosthetic hand function: Normative data, reliability, and validity," Arch. Phys. Med. Rehabil., vol. 83, no. 6, pp. 776-783, Jun. 2002.

[72] R. C. Lyle, "A performance test for assessment of upper limb function in physical rehabilitation treatment and research.," Int. J. Rehabil. Res., vol. 4, no. 4, pp. 483-92, Jan. 1981.

[73] M. McDonnell, "Action research arm test.," Aust. J. Physiother., vol. 54, no. 3, p. 220, Jan. 2008.

[74] S. a Dalley, D. a Bennett, and M. Goldfarb, "Preliminary functional assessment of a multigrasp myoelectric prosthesis.," Conf. Proc. IEEE Eng. Med. Biol. Soc., vol. 2012, pp. 4172-5, Aug. 2012.

[75] E. D. de Bruin, D. Schoene, G. Pichierri, and S. T. Smith, "Use of virtual reality technique for the training of motor control in the elderly. Some theoretical considerations.," Z. Gerontol. Geriatr., vol. 43, no. 4, pp. 229-34, Aug. 2010.

[76] J. J. LaViola, "A discussion of cybersickness in virtual environments," ACM SIGCHI Bull., vol. 32, no. 1, pp. 47-56, Jan. 2000.

[77] H. Akiduki, S. Nishiike, H. Watanabe, K. Matsuoka, T. Kubo, and N. Takeda, "Visual-vestibular conflict induced by virtual reality in humans," Neurosci. Lett., vol. 340, no. 3, pp. 197-200, Apr. 2003.

[78] M. Ortiz-catalan, S. Nijenhuis, K. Ambrosch, T. B. Eerdt, S. Koenig, and B. Lange, "Virtual Reality," in Emerging Therapies in Neurorehabilitation, vol. 4, J. L. Pons and D. Torricelli, Eds. Berlin, Heidelberg: Springer Berlin Heidelberg, 2014, pp. 249-265.

[79] H. Rehbaum, N. Jiang, and D. Farina, "A new software environment for highdensity emg recording, real time analysis and online feedback," in XIX Congress of the International Society of Electrophysiology and Kinesiology, 2012.

[80] Google Inc., "Android Metrics and Grids." [Online]. Available: http://developer.android.com/design/style/metrics-grids.html.

[81] W. Hamilton, Elements of quaternions. 1866.

[82] N. Jiang, H. Rehbaum, I. Vujaklija, B. Graimann, and D. Farina, "Intuitive, Online, Simultaneous and Proportional Myoelectric Control Over Two Degrees of Freedom in Upper Limb Amputees.," IEEE Trans. Neural Syst. Rehabil. Eng., Aug. 2013. 
[83] N. Jiang, I. Vujaklija, H. Rehbaum, B. Graimann, and D. Farina, "Is Accurate Mapping of EMG Signals on Kinematics Needed for Precise Online Myoelectric Control?," IEEE Trans. Neural Syst. Rehabil. Eng., Oct. 2013.

[84] J. Hahne, F. Biessmann, N. Jiang, H. Rehbaum, D. Farina, F. Meinecke, K. Muller, and L. Parra, "Linear and non-linear regression techniques for simultaneous and proportional myoelectric control," IEEE Trans. Neural Syst. Rehabil. Eng., pp. 1-1, 2014.

[85] A. Stango, F. Negro, and D. Farina, "Spatial Correlation of High Density EMG Signals Provides Features Robust to Electrode Number and Shift in Pattern Recognition for Myocontrol," IEEE Trans. Neural Syst. Rehabil. Eng., vol. submitted, 2014.

[86] I. Vujaklija, "Minimally Supervised Algorithm for Advanced and Intuitive MyoControl of Upper Limb Prosthesis based on a Probabilistic Latent Variable Model Approach," Lübeck University / FH Lübeck, 2013.

[87] P. Fitts, "The information capacity of the human motor system in controlling the amplitude of movement.," J. Exp. Psychol., 1954.

[88] I. MacKenzie, "A note on the information-theoretic basis for Fitts' law," J. Mot. Behav., 1989.

[89] S. Muceli, N. Jiang, and D. Farina, "Extracting Signals Robust to Electrode Number and Shift for Online Simultaneous and Proportional Myoelectric Control by Factorization Algorithms.," IEEE Trans. Neural Syst. Rehabil. Eng., Oct. 2013.

[90] S. Muceli, A. T. Boye, A. Avella, D. Farina, and A. D'Avella, "Identifying Representative Synergy Matrices for Describing Muscular Activation Patterns During Multidirectional Reaching in the Horizontal Plane," J. Neurophysiol., vol. 103, no. 3, pp. 1532-1542, Mar. 2010.

[91] N. Jiang, "Extracting force functions from the multi-channel surface electromyographic signal," UNIVERSITY OF NEW BRUNSWICK, 2009.

[92] F. a Mussa-Ivaldi and E. Bizzi, "Motor learning through the combination of primitives.," Philos. Trans. R. Soc. Lond. B. Biol. Sci., vol. 355, no. 1404, pp. 1755-69, Dec. 2000.

[93] E. Bizzi, a. D’Avella, P. Saltiel, and M. Tresch, "Book Review: Modular Organization of Spinal Motor Systems," Neurosci., vol. 8, no. 5, pp. 437-442, Oct. 2002.

[94] A. d'Avella, P. Saltiel, and E. Bizzi, "Combinations of muscle synergies in the construction of a natural motor behavior.," Nat. Neurosci., vol. 6, no. 3, pp. 300-8, Mar. 2003. 
[95] R. Merletti and P. A. Parker, Electromyography: Physiology, engineering, and noninvasive applications, vol. 11. Wiley-IEEE Press, 2004.

[96] D. Farina and R. Merletti, "A novel approach for precise simulation of the EMG signal detected by surface electrodes.," IEEE Trans. Biomed. Eng., vol. 48, no. 6, pp. 637-46, Jun. 2001.

[97] D. Farina and a Rainoldi, "Compensation of the effect of sub-cutaneous tissue layers on surface EMG: a simulation study.," Med. Eng. Phys., vol. 21, no. 6-7, pp. 487-97, 1999.

[98] D. Farina, L. Mesin, S. Martina, and R. Merletti, "A surface EMG generation model with multilayer cylindrical description of the volume conductor.," IEEE Trans. Biomed. Eng., vol. 51, no. 3, pp. 415-26, Mar. 2004.

[99] D. Farina, L. Mesin, S. Martina, and R. Merletti, "Comparison of spatial filter selectivity in surface myoelectric signal detection: influence of the volume conductor model.," Med. Biol. Eng. Comput., vol. 42, no. 1, pp. 114-20, Jan. 2004.

[100] D. Seung and L. Lee, "Algorithms for non-negative matrix factorization," Adv. Neural Inf. Process. Syst., 2001.

[101] A. d Avella and M. Tresch, "Modularity in the motor system: decomposition of muscle patterns as combinations of time-varying synergies," ... neural Inf. Process. Syst., 2002.

[102] V. C. K. Cheung, A. d'Avella, M. C. Tresch, and E. Bizzi, "Central and sensory contributions to the activation and organization of muscle synergies during natural motor behaviors.," J. Neurosci., vol. 25, no. 27, pp. 6419-34, Jul. 2005.

[103] R. Shiavi, C. Frigo, and A. Pedotti, "Electromyographic signals during gait: Criteria for envelope filtering and number of strides," Med. Biol. Eng. Comput., vol. 36, no. 2, pp. 171-178, Mar. 1998.

[104] M. Sartori, M. Reggiani, D. Farina, and D. G. Lloyd, "EMG-driven forwarddynamic estimation of muscle force and joint moment about multiple degrees of freedom in the human lower extremity.," PLoS One, vol. 7, no. 12, p. e52618, Jan. 2012.

[105] D. Farina, "Interpretation of the surface electromyogram in dynamic contractions," Exerc. Sport Sci. Rev., pp. 121-127, 2006.

[106] C. L. Chicoine, A. M. Simon, and L. J. Hargrove, "Prosthesis-guided training of pattern recognition-controlled myoelectric prosthesis," in 2012 Annual International Conference of the IEEE Engineering in Medicine and Biology Society, 2012, vol. 60611, pp. 1876-1879.

[107] E. H. Moore, "On the reciprocal of the general algebraic matrix," Bull. Am. Math. Soc., vol. 26, no. 9, pp. 385-397, Jun. 1920. 
[108] R. Penrose, "A generalized inverse for matrices," Math. Proc. Cambridge Philos. Soc., vol. 51, no. 03, pp. 406-413, Oct. 1955.

[109] A. D. Roche, H. Rehbaum, D. Farina, and O. C. Aszmann, "Prosthetic Myoelectric Control Strategies: A Clinical Perspective," Curr. Surg. Reports, vol. 2, no. 3, p. 44, Jan. 2014.

[110] E. a. Corbett, E. J. Perreault, and T. a. Kuiken, "Comparison of electromyography and force as interfaces for prosthetic control," J. Rehabil. Res. Dev., vol. 48, no. 6, p. 629, 2011.

[111] W. Kruskal and W. Wallis, "Use of ranks in one-criterion variance analysis," J. Am. Stat. ..., vol. 47, no. 260, pp. 583-621, 1952.

[112] H. Rehbaum, N. Jiang, I. Vujaklija, A. Holobar, and D. Farina, "Deeper Insights into Muscles after TMR - Visualization, Online Feedback and Physiological Understandings using High Density Electromyography," in 1st International TMR Symposium, 2013.

[113] D. Farina, H. Rehbaum, A. Holobar, I. Vujaklija, N. Jiang, C. Hofer, S. Salminger, H.-W. Van Vliet, and O. Aszmann, "Non-Invasive, Accurate Assessment of the Behavior of Representative Populations of Motor Units in Targeted Reinnervated Muscles," IEEE Trans. Neural Syst. Rehabil. Eng., pp. $1-1,2014$.

[114] R. V Kenyon, J. Leigh, and E. a Keshner, "Considerations for the future development of virtual technology as a rehabilitation tool.," J. Neuroeng. Rehabil., vol. 1, no. 1, p. 13, Dec. 2004.

[115] H. Rehbaum, "Automatic hand gesture recognition from multichannel surface electromyography," RWTH Aachen University, 2011.

[116] D. Farina, N. Jiang, H. Rehbaum, A. Holobar, B. Graimann, H. Dietl, and O. Aszmann, "The Extraction of Neural Information from the Surface EMG for the Control of Upper-Limb Prostheses: Emerging Avenues and Challenges," IEEE Trans. Neural Syst. Rehabil. Eng., vol. accepted, pp. 1-1, 2014. 


\section{Annex I: Literature research}

Before the start of this $\mathrm{PhD}$ project, as well as during the project period, a literature research has been performed. Since already my diploma thesis was on the topic of myoelectric control [115], I started with an existing database of publications on this topic.

Starting from this database, I used different methods to follow updates in the scientific community and keep up to the latest developments:

I. Using PubMed I configured an alert for the following search tags and reviewed them on bi-weekly basis:

a. Prosthetic control

b. Gesture recognition

c. Adaptive prosthesis

d. (emg OR myoelectric) AND (classifier OR classification)

e. adaptive AND (prosthesis OR prostheses OR prosthetic) AND (hand OR upper OR arm OR transradial)

II. Additionally to the publications listed in PubMed, I also followed the most important research groups for myoelectric control of upper limb prostheses through direct contact or their websites.

III. Through regular scientific meetings and journal clubs within the Department of Neurorehabilitation Engineering as well as the AMYO Project Consortium, I received additional input from peers and colleagues of different research fields. 
IV. I reviewed several manuscripts for the following scientific journals:

a. IEEE Transactions on Biomedical Engineering

b. IEEE Journal of Biomedical and Health Informatics

c. IEEE Transactions on Neural Systems \& Rehabilitation Engineering

V. Together with Dr. Aidan Roche, a collaborator at the University of Newcastle, I wrote a review on prosthetic control. While Dr. Roche focused on the clinical aspects, I concentrated on the technical developments since the 1940 and therefore reviewed the literature of the past $60+$ years. The review "Prosthetic Myoelectric Control Strategies: A Clinical Perspective" [109] has been published by the peer-reviewed journal "Current Surgery Reports" in January 2014.

VI. During the PhD project I participated in three international conferences as well as multiple scientific meetings and workshops, allowing me to get into touch with peers from the field and discuss the most recent scientific developments. 


\section{Annex II: List of disseminations}

As part of this PhD project I participated in conferences and workshops to make the results of my work available to the scientific community. Additionally, I published in journal publications or contributed as a co-author.

\section{Conferences/Symposia}

\begin{tabular}{|l|c|}
\hline Poster & $\begin{array}{c}\text { Rehbaum H, Jiang N, Farina D (2012) A new software environment for } \\
\text { high-density emg recording, real time analysis and online feedback. In: } \\
\text { XIX Congress of the International Society of Electrophysiology and } \\
\text { Kinesiology. Brisbane. }\end{array}$ \\
\hline Talk & $\begin{array}{c}\text { Rehbaum H, Jiang N, Paredes L, Amsuess S, Graimann B, Farina D (2012) } \\
\text { Real time simultaneous and proportional control of multiple degrees of } \\
\text { freedom from surface EMG: Preliminary results on subjects with limb } \\
\text { deficiency. In: 2012 Annual International Conference of the IEEE } \\
\text { Engineering in Medicine and Biology Society, pp 1346-1349. IEEE. }\end{array}$ \\
\hline Talk & $\begin{array}{c}\text { Rehbaum H, Jiang N, Vujaklija I, Holobar A, Farina D (2013) Deeper } \\
\text { Insights into Muscles after TMR - Visualization, Online Feedback and } \\
\text { Physiological Understandings using High Density Electromyography. } \\
\text { In: 1st International TMR Symposium. Vienna. }\end{array}$ \\
\hline Co-Author & $\begin{array}{c}\text { Hahne JM, Rehbaum H, Biessmann F, Meinecke FC, Muller K-R, Jiang N, } \\
\text { Farina D, Parra LC (2012) Simultaneous and proportional control of 2D } \\
\text { wrist movements with myoelectric signals. In: 2012 IEEE International } \\
\text { Workshop on Machine Learning for Signal Processing, pp 1-6. IEEE. }\end{array}$ \\
\hline
\end{tabular}

\section{Journal Publications}

\begin{tabular}{|l|l|}
\hline Published & $\begin{array}{c}\text { Jiang N*, Rehbaum H*, Vujaklija I, Graimann B, Farina D (2013) Intuitive, } \\
\text { Online, Simultaneous and Proportional Myoelectric Control Over Two } \\
\text { Degrees of Freedom in Upper Limb Amputees. IEEE Transactions on } \\
\text { Neural Systems and Rehabilitation Engineering }\end{array}$ \\
\hline Published & $\begin{array}{c}\text { Jiang N, Vujaklija I, Rehbaum H, Graimann B, Farina D (2013) Is Accurate } \\
\text { Mapping of EMG Signals on Kinematics Needed for Precise Online } \\
\text { Myoelectric Control ? IEEE Transactions on Neural Systems and } \\
\text { Rehabilitation Engineering }\end{array}$ \\
\hline Published & $\begin{array}{l}\text { Roche AD, Rehbaum H, Farina D, Aszmann OC (2014) Prosthetic } \\
\text { Myoelectric Control Strategies: A Clinical Perspective. Current Surgery } \\
\text { Reports }\end{array}$ \\
\hline
\end{tabular}




\begin{tabular}{|c|c|}
\hline Published & $\begin{array}{c}\text { Farina D, Rehbaum H, Holobar A, Vujaklija I, Jiang N, Hofer C, Salminger S, } \\
\text { Aszmann OC (2014) Non-Invasive, Accurate Assessment of the Behavior } \\
\text { of Representative Populations of Motor Units in Targeted Reinnervated } \\
\text { Muscles. IEEE Transactions on Neural Systems and Rehabilitation } \\
\text { Engineering }\end{array}$ \\
\hline Published & $\begin{array}{c}\text { Farina D, Jiang N, Rehbaum H, Holobar A, Graimann B, Dietl H, Aszmann } \\
\text { OC (2014) The Extraction of Neural Information from the Surface EMG } \\
\text { for the Control of Upper-Limb Prostheses: Emerging Avenues and } \\
\text { Challenges. IEEE Transactions on Neural Systems and Rehabilitation } \\
\text { Engineering }\end{array}$ \\
\hline Published & $\begin{array}{c}\text { Hahne JM, Biessmann F, Jiang N, Rehbaum H, Farina D, Meinecke FC, } \\
\text { Müller K-R, and Parra LC (2014) Linear and non-linear regression } \\
\text { techniques for simultaneous and proportional myoelectric control, IEEE } \\
\text { Transactions on Neural Systems and Rehabilitation Engineering }\end{array}$ \\
\hline Submitted & $\begin{array}{c}\text { Rehbaum H, Farina D, Adaptive common average filtering for myocontrol } \\
\text { applications. Medical \& biological engineering \& computing. }\end{array}$ \\
\hline
\end{tabular}

